\title{
Feature Interaction in Web Services
}

\author{
By \\ Yun Luo
}

\author{
A thesis submitted to the \\ School of Computer Science \\ and the Faculty of Graduate Studies and Research \\ in partial fulfillment of the requirements \\ for the degree of \\ Master of Computer Science \\ Ottawa-Carleton Institute for Computer Science \\ School of Computer Science \\ Carleton University \\ Ottawa, Ontario
}

December 2004

(C) Copyright 2004, Yun Luo 


$\begin{array}{ll}\begin{array}{l}\text { Library and } \\ \text { Archives Canada }\end{array} & \begin{array}{l}\text { Bibliothèque et } \\ \text { Archives Canada }\end{array} \\ \begin{array}{l}\text { Published Heritage } \\ \text { Branch }\end{array} & \begin{array}{l}\text { Direction du } \\ \text { Patrimoine de l'édition }\end{array} \\ \begin{array}{l}\text { 395 Wellington Street } \\ \text { Ottawa ON K1A ON4 } \\ \text { Canada }\end{array} & \begin{array}{l}\text { 395, rue Wellington } \\ \text { Ottawa ON K1A ON4 } \\ \text { Canada }\end{array}\end{array}$

Your file Votre référence ISBN: 0-494-00762-1

Our file Notre référence

ISBN: 0-494-00762-1

NOTICE:

The author has granted a nonexclusive license allowing Library and Archives Canada to reproduce, publish, archive, preserve, conserve, communicate to the public by telecommunication or on the Internet, loan, distribute and sell theses worldwide, for commercial or noncommercial purposes, in microform, paper, electronic and/or any other formats.

The author retains copyright ownership and moral rights in this thesis. Neither the thesis nor substantial extracts from it may be printed or otherwise reproduced without the author's permission.
AVIS:

L'auteur a accordé une licence non exclusive permettant à la Bibliothèque et Archives Canada de reproduire, publier, archiver, sauvegarder, conserver, transmettre au public par télécommunication ou par l'Internet, prêter, distribuer et vendre des thèses partout dans le monde, à des fins commerciales ou autres, sur support microforme, papier, électronique et/ou autres formats.

L'auteur conserve la propriété du droit d'auteur et des droits moraux qui protège cette thèse. $\mathrm{Ni}$ la thèse ni des extraits substantiels de celle-ci ne doivent être imprimés ou autrement reproduits sans son autorisation.
In compliance with the Canadian

Privacy Act some supporting forms may have been removed from this thesis.

While these forms may be included in the document page count, their removal does not represent any loss of content from the thesis.
Conformément à la loi canadienne sur la protection de la vie privée, quelques formulaires secondaires ont été enlevés de cette thèse.

Bien que ces formulaires aient inclus dans la pagination, il n'y aura aucun contenu manquant. 


\section{Abstract}

Although the undesirable feature interaction problem has been first studied in the telecommunication domain, it is nothing new in the component-based systems and service-oriented systems beyond telecommunication. With the rapid evolution of Web services, this problem also becomes increasingly inevitable and critical in Web service composition. In this thesis, first, a classification for a collection of undesirable feature interactions in the Web service domain will be presented by causes and three nature dimensions: type, scope and role. It may help us to distinguish whether a particular approach for managing the undesirable feature interaction has been addressed in this domain. Then a detection approach will be presented for detecting feature interactions at the requirements stage in this domain with the combination of Goal-oriented Requirement Language (GRL) and Use Case Maps (UCM), which are respectively for non-functional requirements and scenarios. This approach is based on goal-oriented analysis, which allows us to reason about feature interactions in terms of goal conflicts. An e-store system will serve as a case study for giving a re-examination about the classification and how the detection methodology will work. It also will prove the existence of the feature interaction problem in the Web service domain. 


\section{Acknowledgements}

First of all I would like to express my sincere thanks to my supervisors Dr. Michael

Weiss and Dr. Babak Esfandiari, for their valuable suggestions, profound guidance, and financial support for the FIW'03 conference in Ottawa. I also want to thank them for their numerous comments on drafts of this work, and longstanding commitment to make this thesis a reality.

Deep appreciation is expressed to Dr. Daniel Wu for many helpful discussions and comments.

Deep gratitude is expressed to Steven Lobo and WTS tutors for their roles in reviewing this thesis during final preparations.

I also would like to thank my family and all other friends who gave me help during my graduate studies for their support and encouragement. 


\section{Contents}

Abstract iii

Acknowledgements iv

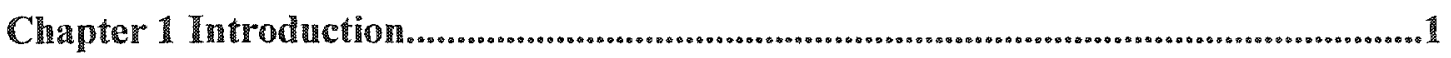

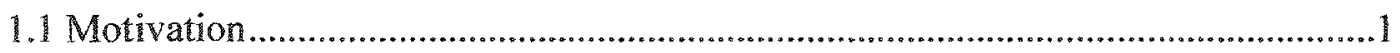

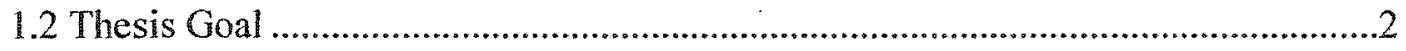

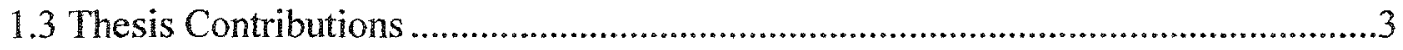

1.4 Thesis Organization ...................................................................................... 4

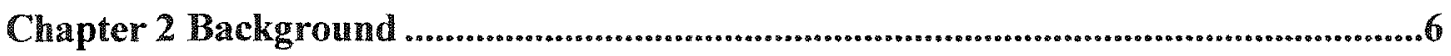

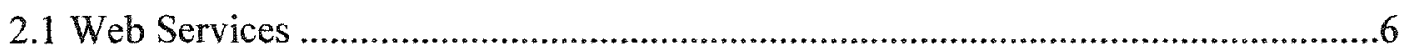

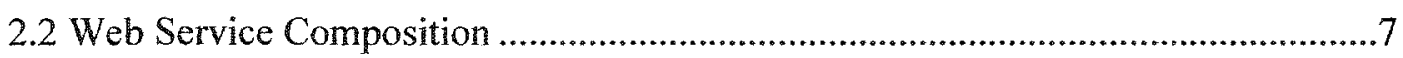

2.2.1 Web Service Composition Languages ....................................................

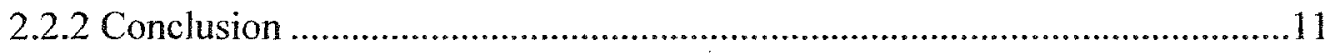

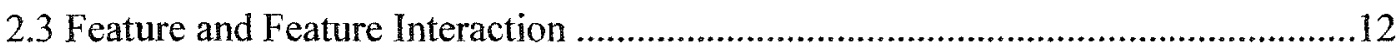

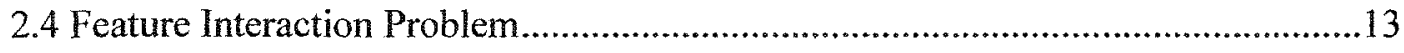

2.5 Approaches to Solving the Feature Interaction Problem ...................................14

2.6 Feature Interaction in Web Services .................................................................. 17

2.7 Chapter Summary .......................................................................................20

Chapter 3 Goal-oriented Requirement Language and Use Case Maps....................21

3.1 Use Case Maps...........................................................................................21

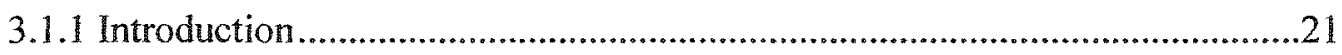

3.1.2 UCM Notation Elements..................................................................22

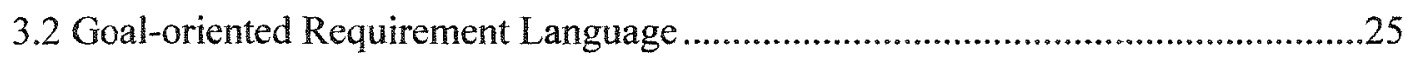

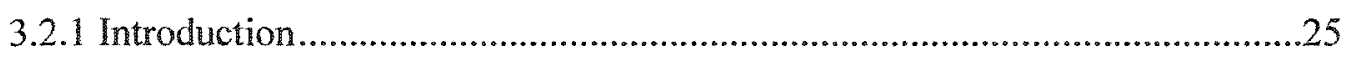

3.2.2 GRL Notation Elements..................................................................26

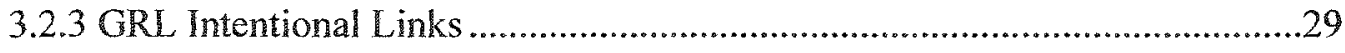

3.2.4 Reasoning about Design Alternatives .......................................................34

3.3 Chapter Summary .......................................................................................36

Chapter 4 Feature Interaction Categories and a Detection Approach ......................37 
4.1 Three Dimensions for Categories in Web Services

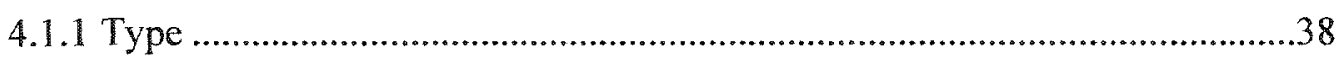

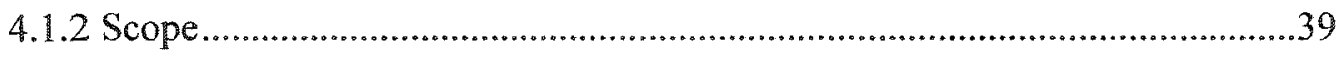

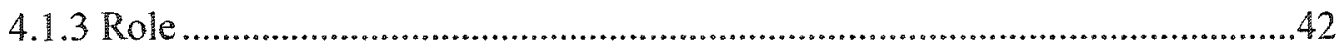

4.2 Categorization of the Cause of Interaction .....................................................43

4.2.1 Violation of Assumptions about Services....................................................4 43

4.2.2 Different Invocation Order .......................................................................44

4.2.3 Resource Contention............................................................................44

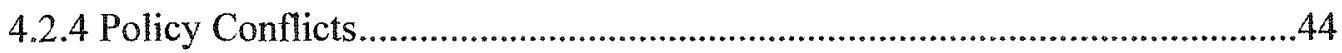

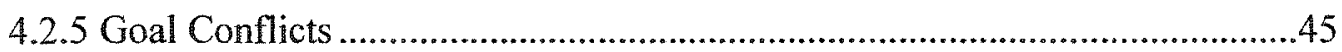

4.2.6 Deployment and Ownership ...............................................................45

4.2.7 Encapsulation and Information Hiding ....................................................46

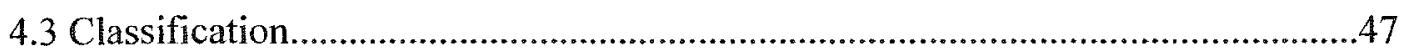

4.3.1 FISR (Functional-Internal-SingleRole Feature Interaction) ...................... 53

4.3.2 FIMR (Functional-Internal-MultipleRoles Feature Interaction).................. 55

4.3.3 FESR (Functional-External-SingleRole Feature Interaction) ...................... 59

4.3.4 FEMR (Functional-External-MultipleRoles Feature Interaction) ................60 60

4.3.5 NFISR (Non-Functional-Internal-SingleRole Feature Interaction) ............ 62

4.3.6 NFIMR (Non-Functional-Internal-MultipleRoles Feature Interaction)....... 63

4.3.7 NFESR (Non-Functional-External-SingleRole Feature Interaction)............ 65

4.3.8 NFEMR (Non-Functional-External-MultipleRoles Feature Interaction) ......66

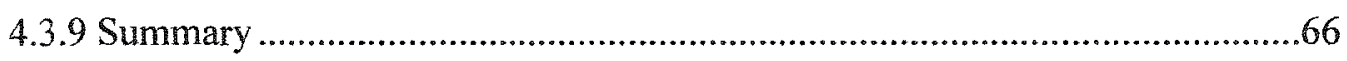

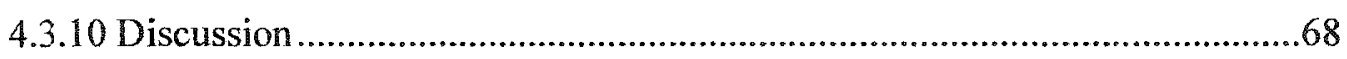

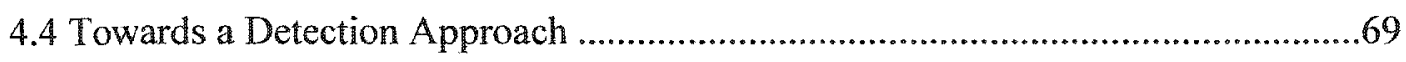

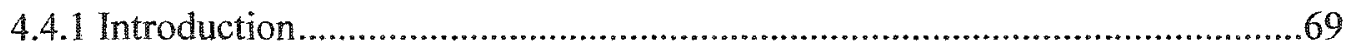

4.4.2 Towards a Detection Approach ...........................................................70

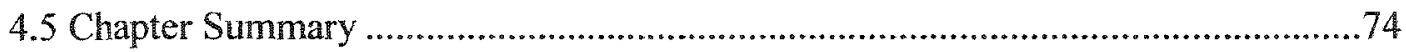

Chapter 5 Case Study: an E-Store Example ........................................................... 76

5.1 The E-Store System Functionality ..................................................................... 76

5.2 The E-Store System Analysis .......................................................................78 
5.2.1 A High-Level GRL Model of the E-Store System.....................................78

5.2.2 Root Use Case Map of the E-Store System ..............................................80

5.2.3 A Refined GRL Model of the E-Store System ........................................82

5.2.4 A Refined Use Case Map model for the E-Store System ...........................83

5.2 .5 Web services in the E-Store System ..................................................84

5.3 Feature Interactions in the E-Store System..................................................8

5.3.1 E-Store Login Function......................................................................8 8

5.3.2 Search \& Display Function....................................................................101

5.3.3 Order Processing Function....................................................................110

5.3.4 Billing \& Shipping Function............................................................ 117

5.4 Chapter Summary ....................................................................................... 128

Chapter 6: Conclusion and Future Work

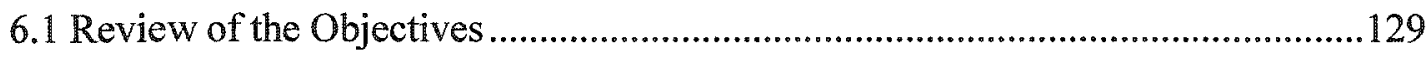

6.2 Summary of the Contributions........................................................................ 130

6.3 Future Work ..................................................................................... 131

Appendix:The Index of Feature Interactions in Web Services ............................... 133

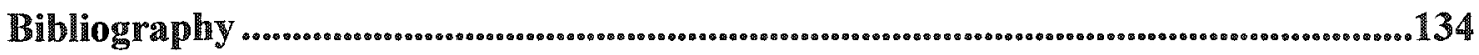




\section{LIST OF TABLES}

Table 4.1: Eight Categories of the Feature Interactions in Web services................47

Table 4.2: The Relationship between Examples and Categories and Causes.............66

Table 4.3: The Summary of the Relationship between Causes and Categories..........67 


\section{LIST OF FIGURES}

Figure 2.1: Web Service Architecture ........................................................................................7

Figure 2.2: Overview of Web Services Technology .................................................................8

Figure 3.1: UCM Basic Notation......................................................................................22

Figure 3.2: (a) OR-join(b) OR-fork ……….............................................................23

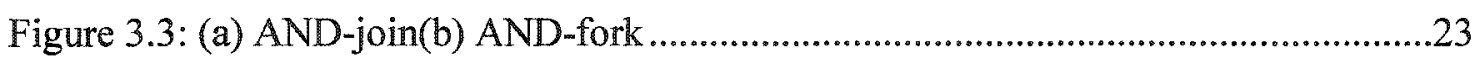

Figure 3.4: Static Stubs have only One Plug-in.................................................................23

Figure 3.5: Dynamic Stubs may have Multiple Plug-in .....................................................24

Figure 3.6: Using UCM to Analyze the Deployment and Ownership Issue in an E-Store

Login Example ...........................................................................................25

Figure 3.7: Goal Personalization.....................................................................................26

Figure 3.8: Soft-goal Privacy .......................................................................................27

Figure 3.9: Task Identity Management...............................................................................27

Figure 3.10: An Actor E-Store with a Task Login.............................................................28

Figure 3.11: Belief Example .................................................................................................29

Figure 3.12: Resource Bandwidth..................................................................................29

Figure 3.13: Goal Means-ends Structure ........................................................................30

Figure 3.14: Task Decomposition Structure .......................................................................30

Figure 3.15: Contribution Relationship Graphical Notation.................................................31

Figure 3.16: Soft-Goal Contribution Structure ....................................................................32

Figure 3.17: Correlations Links Graphical Notation ............................................................32

Figure 3.18: Model Structure Series Connected with Correlation........................................32

Figure 3.19: Actor Client depends on Actor E-Store on Login ..........................................33

Figure 3.20: Goal Conflicts in the Word Processing Example .............................................35

Figure 3.21: Consult with Initiator before Invoking the Spellchecking .............................35

Figure 4.1: An Internal Feature Interaction between Features Transaction Management and Logging ..................................................................................................40 ix 
Figure 4.2: An External Feature Interaction between Features Search Request Cache and Book Search

Figure 4.3: The Architecture of the E-Book Access System ........................................48

Figure 4.4: The GRL Model for the E-Book Access System ..........................................49

Figure 4.5: The GRL Model for an Interaction between Transaction Management and Logging

Figure 4.6: The GRL Model for an Interaction between Logging for Billing and Cache .56

Figure 4.7: The GRL Model for an Interaction between ContentGenerator and

Spellchecker. .58

Figure 4.8: The GRL Model for an Interaction between the Features Search Request

Cache and Book Search .61

Figure 4.9: The GRL Model for an Interaction between Manageability and Trust..........64

Figure 4.10: Steps of the Feature Interaction Detection Approach ..................................71

Figure 4.11: The Web services-QoS stack............................................................... 74

Figure 5.1: The Architecture of the E-Store System...................................................78

Figure 5.2: The High-Level GRL Model of E-Store System.........................................79

Figure 5.3: The Root Use Case Map of the E-Store System ..........................................80

Figure 5.4: A Refined GRL Model of the E-Store System...........................................83

Figure 5.5: A Refined Scenario Mapping of the E-Store System....................................84

Figure 5.6: Force Hierarchy for Web Services Usability .............................................89

Figure 5.7: Force Hierarchy for Web Services Privacy ..................................................90

Figure 5.8: Using Two Services to Improve the Usability of the System .........................91

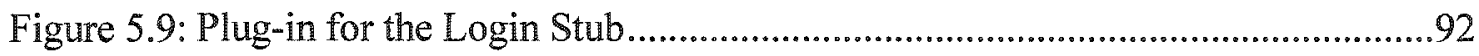

Figure 5.10: Non-Functional Interaction between Privacy and Usability .......................93

Figure 5.11: Set the .NET Passport Work Mode to Test Mode (Site ID = 1) ................ 94

Figure 5.12: Runs the Test Site.........................................................................95

Figure 5.13: Enters the Email Address and Password, and Clicks "Sign in" Button .......96

Figure 5.14: The User (service consumer) should not have Gotten his/her Profile from the Test Site (service provider) ..........................................................97

Figure 5.15: Using Use Case Map to Analyze the Deployment Issue...........................98 
Figure 5.16: Refined Use Case Map to Resolve the Deployment Issue

Figure 5.17: Decouple the Authentication and Authorization Features to Avoid the

Feature Interaction .100

Figure 5.18: Force Hierarchy for Performance. 102

Figure 5.19: Force Hierarchy for Maintainability. .102

Figure 5.20: Force Hierarchy for Interoperability 104

Figure 5.21: Use Case Map for the Search \& Display Function 104

Figure 5.22: Feature Interactions among Interoperability, Maintainability, and Performance 106

Figure 5.23: Using Use Case Map to Analyze Deployment Issue. 107

Figure 5.24: Refined the Use Case Map 108

Figure 5.25: Using Vocabulary and Service Check to Avoid the Feature Interaction ....109

Figure 5.26: Use Case Map for the Order Processing Function .... 112

Figure 5.27: Order Processing Produces Infinite Loops and Reduces the Performance .113

Figure 5.28: Using UCM to Analyze the Deployment Issue 114

Figure 5.29: Refined Use Case Map. 115

Figure 5.30: The Solution for Feature Interaction .116

Figure 5.31: Force Hierarchy for Profitability . .118

Figure 5.32: Force Hierarchy for Correctness 119

Figure 5.33: Use Case Map for the Billing \& Shipping Function 119

Figure 5.34: Feature Interactions in Billing \& Shipping Function.... 121

Figure 5.35: Another Feature Interaction in Billing \& Shipping Function 123

Figure 5.36: Applying the new policy can Avoid the Feature Interaction. 125

Figure 5.37: Refined Use Case Map for the Billing \& Shipping Function. 125 


\section{List of Acronyms}

\begin{tabular}{|c|c|}
\hline $\mathrm{AP}$ & Access Profile \\
\hline AWS & Amazin Web Service \\
\hline BPEL4WS & Business Process Execution Language for Web Services \\
\hline BPML & Business Process Modeling Language \\
\hline BPSS & Business Process Schema Specification \\
\hline BS & Boogle Search \\
\hline BSS & Boogle Search Service \\
\hline CDP & Check Discount Policies \\
\hline CES & Currency Exchange Service \\
\hline CLIQ & Check Local Item Quantities \\
\hline $\mathrm{CO}$ & Check Out \\
\hline CRIQ & Check Remote Item Quantities \\
\hline GD & Group Discount \\
\hline GRL & Goal-oriented Requirement Language \\
\hline HTTP & Hypertext Transfer Protocol \\
\hline $\mathbf{M}$ & Identity Management \\
\hline IMS & Identity Management Service \\
\hline LOPS & Local Order Process Service \\
\hline LS & Local Seare最 \\
\hline $\mathbb{L S S}$ & Local Search Service \\
\hline
\end{tabular}

xii 
NPS .Net Passport Service

P3P Platform for Privacy Preferences

PDS Payment Discount Service

PMS Profile Management Service

PLO Place Local Order

PO Process Order

QoS Quality of Service

ROP Remote Order Process

ROPCS Remote Order Process Checker Service

ROPS Remote Order Process Service

RS Remote Search

RSC Remote Service Checker

RSS Remote Search Service

SCS Search Checker Service

SOA Service-Oriented Architecture

SOAP Simple Object Access Protocol

SS Shipping Service

TC Tax Calculation

TS Tax Service

UCM Use Case Maps

UDDI Universal Description Discovery and Integration xiii 
URN User Requirements Notation

W3C World Wide Web Consortium

WSCI Web Service Choreography Interpace

WSDL Web Services Description Language

WSFL Web Services Flow Language

XLANG Web Services for Business Process Design

XML $\quad$ Extensible Markup Language

xiv 


\section{Chapter 1 Introduction}

\subsection{Motivation}

Web services are being developed and deployed by different vendors and organizations everyday. Some of them may work as individual services, for example, a Stock Quote Lookup Service; others may need to be composed with other services to carry out certain kinds of interactions. For example, a Travel service may need to compose Car Rental services, Hotel services, and Airline services. Current research mainly focuses on how to compose Web services. Different vendors offer different standards and solutions for Web service composition, such as BPEL4WS (Business Process Execution Language for Web Services) [Curbera02], WSFL (Web Services Flow Language) [WSFL01], XLANG (Web Services for Business Process Design) [XLANG01], WSCI (Web Service Choreography Interface) [WSCI02], BPSS (Business Process Schema Specification) [BPSS02] and BPML (Business Process Modeling Language) [BPML02]. These composition languages provide several approaches to improve the quality of Web service composition. For example, BPEL4WS ensures the interoperability across the compositions by defining a model and a grammar for describing the behavior of a business process based on interactions among Web services. Furthermore, these approaches can be used to avoid some undesirable feature interactions though they are not specifically designed to do so. However, this function is limited because those approaches only concentrate on business process of the static and functional composition. They do not have enough support of dynamic Web service composition and the non-functional concerns. They 
do not avoid the undesirable feature interactions occurring among the dynamic composition and non-functional concerns. In other words, the undesirable feature interaction problem during Web service composition has not been explored thoroughly.

The concepts feature and feature interaction originated in the telecommunication domain. A feature is the minimum user-visible service unit [ITU-T92] in this domain. In the Web service domain, it can be a function in a Web service, or a Web service itself, or a functional unit composed by several Web services. A feature interaction means that a feature invokes or influences another feature directly or indirectly. More details about their definitions will be introduced in Chapter 2 . The feature interaction problem is a side effect during Web service composition. It has been first investigated in the telecommunication domain. However, it may also occur in the Web service domain. Because of the nature of Web services and Internet, this problem may become more pervasive and serious than in telecommunications systems. Hence, a feature interaction classification and a relative detection approach will be necessary and useful to detect and prevent the feature interaction problem in the Web service domain.

\subsection{Thesis Goal}

The first goal of this thesis is to classify a collection of undesirable feature interactions in Web services by causes and three nature dimensions: type, scope, and role. This classification aims to improve understanding of the scope of the feature interaction problem in the Web service domain. 
The second goal of this thesis is to propose a further step towards detection approach for feature interactions at the requirements stage in Web services, extending the previous work by [W\&E04] with the combination of Goal-oriented Requirement Language (GRL) and Use Case Maps (UCM) frameworks. GRL is used to analyze goals and nonfunctional requirements in a system, and UCM is used to analyze scenarios, functional requirements, and deployment issues.

In support of the classification and the detection approach, an e-store system will be presented as a case study in Chapter 5 .

\subsection{Thesis Contributions}

The major contributions of this thesis are as follows:

- It analyzes and classifies feature interactions in the Web service domain by their nature and causes extension with some related examples.

It proposes a further development for an existing detection approach for feature interactions at the requirements stage in Web services by combining the GRL and UCM frameworks.

It provides an e-store example to illustrate how to use the approach to detect feature interactions in Web services, and to verify the classification of feature interactions in this domain. 
It documents those identified feature interactions in Web services, so they can be referred by Web service application system architects in the future.

\subsection{Thesis Organization}

The rest of the thesis is organized into five parts:

\section{Chapter 2: Background}

This chapter introduces the concepts of feature and feature interaction problem. It will also present a survey of related work on the feature interaction problem in the Web service domain.

\section{Chapter 3: Goal-oriented Requirement Language and Use Case Maps}

This chapter gives an overview of GRL and UCM and some of their notations used in this thesis. It also will explain how the combination of GRL and UCM can be used to detect feature interactions in Web services.

\section{Chapter 4: Feature Interaction Categories and a Detection Approach}

This chapter consists of two parts, a classification of feature interaction in the Web service domain and a further development of a feature interaction detection approach at the requirements stage in this domain. 


\section{Chapter 5: Case Study: An E-Store Example}

This chapter is used to re-examine the classification and the detection approach presented in Chapter 4. The detection approach will be applied to four common functionalities of an e-store system. Feature interactions in this system will be detected and documented.

\section{Chapter 6: Conclusion and Future Work}

This chapter summarizes the manner in which the thesis meets the stated objectives and confirms the claimed contributions. The thesis concludes with a statement of suggested areas for future research. 


\section{Chapter 2 Background}

\subsection{Web Services}

Web services are "self-contained, self-describing modular applications" [Martin01]. There are many definitions [PerfectXml][Systinet][W3C04] of Web services, and the most familiar is [W3C04]:

A Web service is a software system designed to support interoperable machine-tomachine interaction over a network. It has an interface described in a machineprocessable format (specifically WSDL). Other systems interact with the Web service in a manner prescribed by its description using SOAP messages, typically conveyed using HTTP with an XML serialization in conjunction with other Web-related standards.

Web services are based on a service-oriented architecture (SOA), which is essentially a collection of services. There are three basic roles in this architecture: service provider, service broker, and service consumer [W3C04]. Service provider publishes services to a service broker. Service consumer finds required services via a service broker and binds to them (see Figure 2.1). Service broker is not always necessary because service consumer can find and invoke services if it already knows the location of the service provider. 


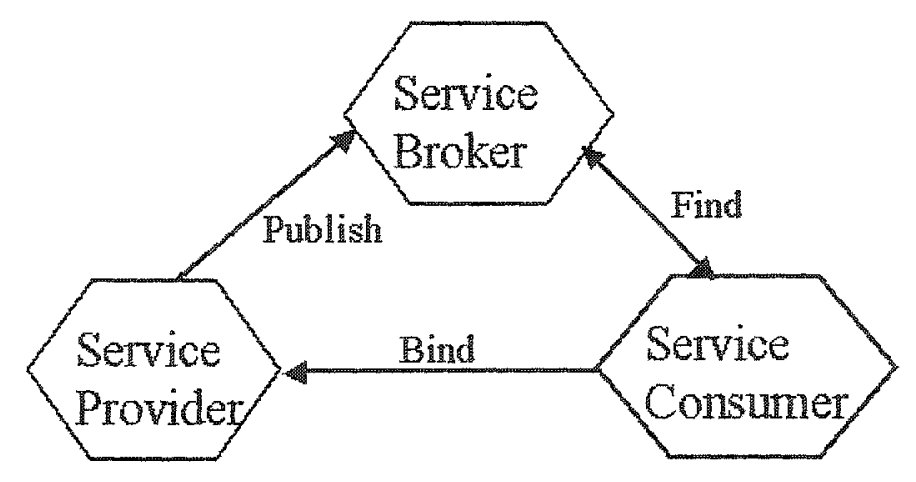

Figure 2.1: Web Service Architecture

In this architecture, service providers advertise and register their services respectively using a standard language called the Web Services Description Language (WSDL) [WSDL04] and Universal Description Discovery and Integration (UDDI) [UDDI04]. Web service consumers also locate the required services using UDDI. The Simple Object Access Protocol (SOAP) [SOAP04] is used to communicate between service providers and service consumers.

\subsection{Web Service Composition}

\subsubsection{Web Service Composition Languages}

Web service composition is a distributed development model. It is used to construct new Web services on top of existing primitive or other composite Web services. It can take place inside or outside an organization. Take an e-business system for example; an organization can implement the local business process of the e-business system by using its own Web services, such as a search service. Meanwhile, this organization can implement a remote business process by finding and binding with other e-business services on the Intemet, which have the same functional requirements with the local. 
In a real enterprise application, if we want to integrate business processes across enterprise boundaries, it is not enough to merely support a simple interaction using standard messages and protocols. We need an explicit process model to handle the longrunning business interactions in the real application. That is the reason that gave rise to Web services composition languages, such as BPEL4WS (Business Process Execution Language for Web Services) [Curbera02]. These composition languages build directly on top of WSDL (see Figure 2.2 [Aalst03]). WSDL messages and XML Schema type definitions provide the data model used by composition language processes. The difference between those composition languages and WSDL is related to the states. WSDL is in essence stateless because the language is not aware of the state in-between operations. However, the state in-between sending and receiving a message in a requestresponse or solicit-response operation need to be supported, because the state can tell the process what should/can be done. Hence, in order to enable long-lived business transactions in a real enterprise application, any composition technology will have to record those states for dealing with complex service interaction.

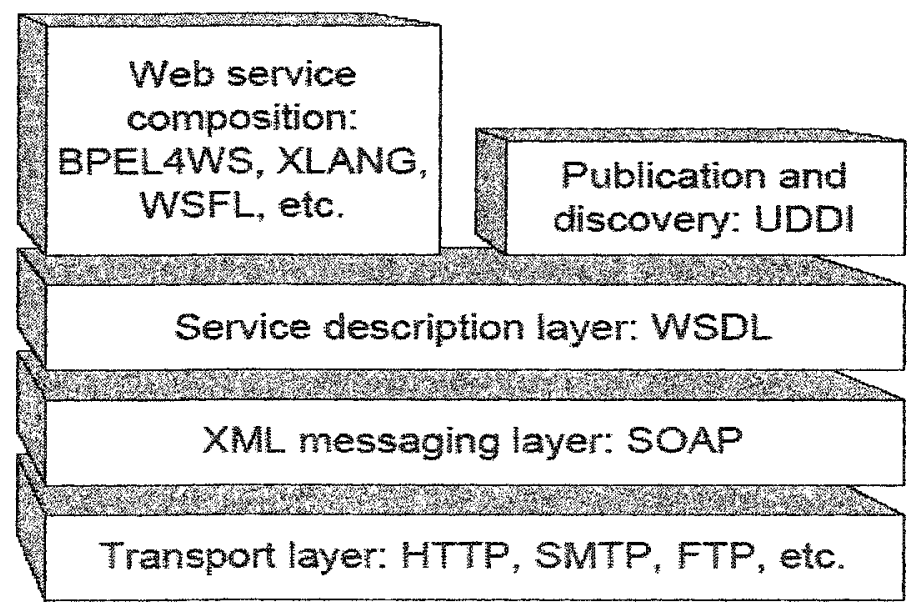

Figure 2.2: Overview of Web Services Technology 
In what follows is a description explained at those composition languages:

WSFL: It is an XML language for the description of Web services composition. It supports two types of Web services compositions: flow model and global model [WSFL01].

Flow model means to model compositions following the execution sequence of the functionality provided by the composed Web services. It can especially be used to model business processes or workflows based on Web services. It is also known as flow composition, orchestration, or choreography of Web services.

Global model models those compositions with decentralized or distributed natures. In other words, it models compositions, which provide a description of how the composed Web services interact with each other. It does not provide a specification of an execution sequence.

XLANG: It is a block-structured language with basic control flow structures, such as sequence, switch, while, all, and pick (for race conditions based on timing or external triggers). The basic constituents of its process definition are actions. It is an extension of WSDL, and the four types of WSDL operations (request/response, solicit response, one way, and notification) can be used as XLANG actions. Furthermore, it adds two other kinds of action: timeouts (deadline and duration) and exceptions [XLANG01]. 
BPEL4WS: Its specification builds on IBM's WSFL and Microsoft's XLANG.

It is used to model the behavior of two business processes: executable and abstract processes [BPEL4WS1.1].

Executable processes model actual behaviors of a participant in a business interaction. They can be executed within an organization.

Abstract processes are process descriptions. They are used to specify the mutually visible message exchange behavior between two partners involved in the protocol. They cannot be executed. Abstract processes only deal with protocol-relevant data, which is identified by BPEL4WS's message properties. For example, in a supply-chain protocol, buyer and seller, as a pair of partners, are connected by this protocol. They both have their own abstract processes, which include the definition dealing with public interactions between them and do not define how they handle their internal processes.

* WSCI: It is an XML-based language used to describe the flow of messages exchanged by a Web service in the context of a process. It allows the description of the observable behavior of a Web service in a message exchange. It is another standard introduced for Web services composition by Sun, BEA, SAP, and Intalio [WSCI02].

BPML: "It is a meta-language for the modeling of business processes, just as XML is a meta-language for the modeling of business data. BPML provides an abstracted execution model for collaborative and transactional business 
processes based on the concept of a transactional finite-state machine" [BPMI04].

\subsubsection{Conclusion}

There are many papers about the comparison of these Web service composition languages. Aalst [EIP02] uses a set of patterns to compare Web service composition languages [Aalst04]. However, they are not concerned with the pros and cons for the contents of feature interaction.

As introduced previously, those five Web service composition languages can avoid and resolve some feature interaction problems. For example, those composition languages can prevent several feature interactions from occurring from simple semantic mismatch, such as data types mismatch, vocabulary mismatch, and so forth.

However, those composition languages have several common problems. For example, they do not have strong support for non-functional concerns during the Web service composition. As we know, any functional change may affect nonfunctional concerns. Sometimes, functional changes may result in undesirable nonfunctional interactions. Another problem with these composition languages is that they can handle the composition logic, which is predefined and static. However, they cannot handle the highly dynamic context of Web services. In fact, many feature interactions in the Web service domain occur during the dynamic Web service compositions. 


\subsection{Feature and Feature Interaction}

The concepts of feature and feature interaction originated in the telecommunication domain. A number of papers are published on this subject every year. Seven international feature interaction workshops have been held so far [FIW92] [FIW94] [FIW95] [FIW97] [FIW98] [FIW00] [FIW03]. In this context, a feature is the minimum user-visible service unit [ITU-T92], or a telecom management-based functional unit provided to customers by a telecommunication network [Bellcore91]. Some classical examples of features in telecommunications systems are: Call Waiting (CW), Call Forwarding Busy Line (CFBL), and Call Forwarding Unconditionally (CFU).

In the domain of system composition and systems built from components, a feature is an observable, relatively independent behavior of characteristics of software. In [ESPRIT01], a feature is explained as "any part or aspect of a specification which the user perceives as having a self-contained functional role." By contrast, a feature in component-based systems may be implemented as functional or may have a nonfunctional nature [Pulvermuller01].

In the Web service domain, a Web service may consist of one or several features. A Web service realizes at least one feature. It implements some functionality and has nonfunctional concerns. "Non-functional" applies to everything, which is not implemented directly [Pulvermuller0]]. For example, the usability of the Boogle Search Service is a non-functional concern, which is not implemented directly. A feature may be a functional unit of a Web service; or it is a whole Web service; or it consists of several Web services. For example, a search function in the Boogle Search Service can be treated as a feature. 
A feature interaction occurs when a feature invokes or influences another feature directly or indirectly. Some feature interactions are desirable because they can be used to achieve certain goals and satisfy user needs, while others are undesirable. The undesirable feature interactions may break system stability or state consistency. Even worse, they may lead to the crash of the whole system.

\subsection{Feature Interaction Problem}

Undesirable feature interactions arise when a feature causes the specification to be incomplete, inconsistent, or unimplementable, or causes the overall system behavior to be undesirable [Zave99].

Based on the definition [Zave99], the following situation also can be treated as feature interaction:

Non-associativity of feature composition

Logical inconsistencies

Some forms of nondeterministic behavior

A classical example of non-associativity composition is the combination of feature Call Waiting (CW) and Call Forwarding when Busy (CFB). If a user subscribes to those two features, then what will happen if there is an incoming call? If the network gives priority to feature $\mathrm{CW}$, the $\mathrm{CFB}$ is compromised, and vice versa [Calder02]. We can find several feature interaction examples at component-based systems [Pulvermuller01]. 


\subsection{Approaches to Solving the Feature}

\section{Interaction Problem}

In the telecommunication domain, Cameron and Velthuijsen [C\&V93] introduced a generally accepted categorization of approaches based on the terminology of avoidance, detection, and resolution. Given the growing exponentially number of features, and combinations among features, Keck and Kuehn add management into the list of approaches [K\&K98].

- Avoidance.

Avoidance aims to develop additional guidelines, such as service platforms and service creation environments, to prevent undesirable feature interactions. Keck and Kuehn divide avoidance into two subgroups: structural approaches and procedural approaches [K\&K98]. The first approach is to avoid feature interactions by a suitable system structure, such as architecture and protocols. The second one is to prevent feature interactions through additional guidelines, such as tool supported service definition and specification.

\section{- Detection.}

Not all feature interactions can be prevented for some reasons, such as issues with cost-effectiveness, performance, dynamic creation of personalized features, and different enterprise domains. Therefore, detection approaches will be necessary. They include off-line detection and on-line detection. Off-line detection means the detection is applied at design time on features. On-line detection is applied at 
run time.

In this thesis, the method proposed in Chapter 4 to detect feature interactions is an off-line process at the requirements stage.

\section{- Resolution.}

Resolution approaches mean to find a mechanism to resolve feature interactions once they are detected. They also include off-line and on-line approaches. For example, in [Tocher89], the resolution is to use a reasonable behavior to replace the undesirable one. In [Velthuijsen93], the negotiating agent approach has been represented to resolve feature interactions.

\section{- Management.}

Management approaches aim at managing the combinatorial exploration of the feature interaction problems and concern the whole interaction handling process [K\&K98].

Feature interaction problems can occur at different stages of the software life cycle. In this thesis, in Chapter 4 , we will present a detection process to detect feature interactions at the requirements stage. In the rest of this section, we will limit the following technologies survey to the requirements stage.

Blom et al. [B\&J\&K94] use a methodology for the modular specification of telephony services within first-order linear-time temporal logic. The style of specification is inspired by the version of temporal logic presented in Lamport's TLA (Temporal Logic of Actions). 
Combes and Pickin [C\&P94] propose a method for detecting feature interactions by verifying that service feature requirements are satisfied in a formal specification language model (expressed in SDL) of the network and the service features.

Gibson et al. [Gib97] address how to avoid, detect, and resolve requirements contradictions during the requirements phase. They use some examples to show how good requirements models could resolve the feature interaction problems that arise when combining features.

Aho et al. [Aho98] present an event-based language called Chisel to define requirements. Feature interactions are detected based on the analysis of sequences of events for features. Because of this, it is difficult to represent concurrent behaviors [Aho98].

Because requirements can be added at need, Jonsson et al. [B\&J\&K94] propose an approach to simplify change management by using different levels of abstract logical concepts. Applying this approach, the feature interactions can be avoided by the specifications automatic verification. This approach uses a simple linear-time temporal logic to formulate requirements.

Nakamura et al. [Nakamura00] propose a feature interaction filtering method at the requirements stage based on Use Case Maps (UCM). UCM can be used to describe and reason about the behaviors in systems by a visual notation for scenarios. It will be used to analyze the scenarios and the deployment and ownership issue in the detection approach presented in Chapter 4. There were many more UCM-based approaches for Feature interaction [Amyot00][A\&L\&B\&G99][Bruin00][Buhr98][M\&A01]. Nakamura et al.'s filtering method can screen out some irrelevant feature combinations before the feature 
interaction detection process. In this method, each feature will be characterized in terms of its stub configuration by using the stub plug-in concept of UCM. This method proposes a composition method of different stub configurations in terms of a matrix and gives one of the following verdicts: (a) Feature Interaction (FI, in short) occurs, (b) FI never occurs or (c) FI-prone.

Gibson [Gib01] uses a formal object oriented method for incrementally constructing, validating, and verifying requirements models, which consist of two parts: the model of the system to be built and the model of the system environment.

\subsection{Feature Interaction in Web Services}

Features and feature interactions in the Web service domain have a number of differences from the telecommunication domain. Many of these differences will affect how these features are created, deployed, and how their interactions are detected and managed. For example, in the Web service domain, the features can be implemented and deployed at numerous vendors or organizations, which maybe unaware of each other or even competing, and thus will not generally be prepared to co-operate to resolve feature interactions during the Web service composition. Additionally, Web services will be created by programmers who may not consider feature interaction issues thoroughly, through either ignorance or expediency. If other Web services providers have to interoperate with these Web services, the feature interaction may occur.

The taxonomy in the Web service domain also has differences with the telecommunication domain. Several traditional taxonomies for feature interactions in the telecommunication domain also can be applied to the Web services domain, such as the 
software lifecycle view [Velthuijsen93], the causal view [Cameron94], the

organizational/operational view [Velthuijsen93], and the configuration view

[Cameron94]. However, due to the nature of the Web services system, in particular

[W\&E04]:

- Distributed Functionality

- Lack of centralized control

- Diversity of technology platforms

- Rapid evolution of business environment

a number of additional complications to the problem of creating and deploying features and resolving their interactions have been introduced. Hence, a refined classification that extends traditional taxonomies is necessary for the Web service domain. We will present it in Chapter 4.

Although traditional avoidance, detection, and resolution approaches also can be used in the Web service domain, as we mentioned above, approaches in the Web service domain may have more concerns on deployment and management because we may not know which service will be composed into our system, and who will provide it. In the telecommunication domain, some non-functional requirements such as usability, security, performance, privacy, etc, may not be considered necessary or have not been studied as a whole, but they are very important in the Web service domain. For example, a Word Processing Service uses two third party intermediaries: Spellchecker Service and Formatter Service. The Spellchecker Service sets the language option is UK English. The Formatter Service also uses a third party Spellchecker Service, which may or may not be the same Spellchecker Service. The Formatter Service assumes that US English should be 
used for spell checking. The Word Processing Service improves the maintainability by using two third party intermediaries. However, the Spellchecker Service will be called twice. This will degrade the performance. The second Spellchecker Service will use US language instead of UK language, so it will break the correctness properties of the Word Processing Service. We will say a feature interaction occurs among non-functional concerns correctness, performance, and maintainability [W\&E04]. Those existing approach in the telecommunication domain do not give an efficient method for detecting these non-functional interactions. Hence, additional approaches for feature interactions in the Web service domain are also necessary. GRL can be used to reason about requirements, especially for dealing with non-functional requirements. It provides intentional elements, links, and actors for expressing different types of concepts at the requirements stage. An approach using GRL to detect feature interactions in the Web service domain will be presented in Chapter 4.

Solanki et al. [Solanki04] [M\&A\&H04] gave a theoretical framework using Temporal Logic and an implementation model for validation/verification of services at runtime to avoid undesirable feature interactions between services. This model has been extended to account for semantic Web services.

Weiss and Esfandiari [W\&E04] presented an approach for detecting feature interaction in Web services. Further development of this approach will be presented in Chapter 4 and validated in Chapter 5. 


\subsection{Chapter Summary}

In this chapter, first we reviewed the Web services definition and its architecture. Then we introduced the Web service composition definition and Web services composition languages. We also talked about why we need Web services composition languages.

Features and the feature interaction problem are another important part in this chapter. We reviewed the basic concepts of feature and feature interaction. We also gave an example of feature interaction in the telecommunication domain. Because we will give a feature interaction detection approach at the requirements stage in Chapter 4 , a survey for feature interaction problems at the requirements stage is also presented in this chapter.

We reviewed the approaches for feature interactions in the telecommunication domain in this chapter. Although this thesis aims to analyze the feature interaction in the Web service domain, the approaches in other domains are helpful to analyze the approaches in the Web service domain.

At the end of this chapter, we explained why we needed to classify the feature interaction and need to find new approaches in the Web service domain.

As we presented above, the GRL and UCM framework can be used to detect feature interaction problem in Web services. Thus, the following chapter will cover GRL and UCM in more detail. 


\section{Chapter 3 Goal-oriented Requirement}

\section{Language and Use Case Maps}

In complex systems, it is hard to describe all requirements by a single notation. Therefore, it is often useful to combine several complementary notations. As we mentioned earlier, the Goal-oriented Requirement Language (GRL) [GRL03] is used to support goal-oriented modeling and reasoning, and to guide the architectural design process. Hence, in this thesis, we can use it to analyze the goal graph for feature interactions in Web service domain, and trace them back to particular solutions. Because the Use Case Maps (UCM) [UCM03] notation is used to express the architectural design at each stage of development, we can use it to analyze deployment issues. They belong to User Requirements Notation (URN) [URN03]. In this chapter, we will introduce some basic concepts of GRL and UCM.

\subsection{Use Case Maps}

\subsubsection{Introduction}

Use Case Maps (UCM) [Buhr96][Buhr99][UCM03][Amyot99] is a visual notation for representing scenarios and system functional requirements. It has been used to analyze and design many different systems, such as real-time systems, object-oriented systems, telecommunication systems, distributed systems, and agent systems.

Its notation is used to illustrate causal relationships among responsibilities by scenario paths. UCM combines behaviors and structures. This combination enables 
reasoning about architecture. UCM uses notations to structure the scenarios. That will make it reasonable to detect potentially undesirable feature interactions among scenarios. Furthermore, UCM provides dynamic (run-time) refinement capabilities, which allow the requirements of systems to be decided at runtime.

\subsubsection{UCM Notation Elements}

In this section, we will just present those UCM notation elements that will be used in this thesis. You can find a full list in [Amyot99].

Basic notation and interpretation

The basic notation just includes scenario paths and responsibilities along these paths (See Figure 3.1).

- Start points (filled circles representing preconditions or triggering causes)

- Responsibilities (crosses, representing actions, tasks, or functions to be performed)

- End points (bars representing post-conditions or resulting effects)

- Components (rectangles representing the entities or objects composing the system)

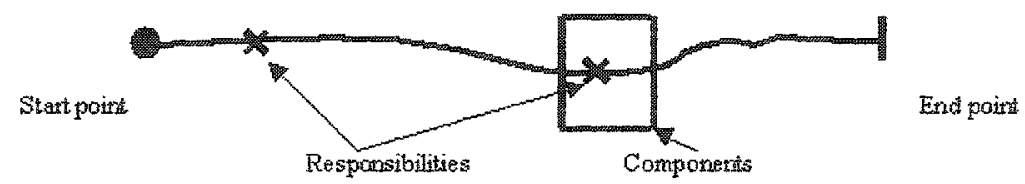

Figure 3.1: UCM Basic Notation 
Shared routes and OR-forks/joins (see Figure 3.2)

OR-join merges two (or more) overlapping paths. OR-fork splits a path into two (or more) alternatives.

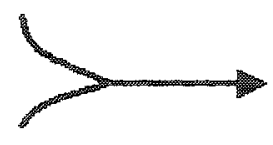

Figure 3.2: (a) OR-join

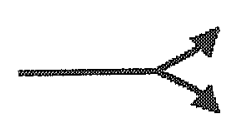

(b) OR-fork

Concurrent routes with AND-forks/joins (see Figure 3.3)

An AND-join synchronizes two paths while an AND-fork splits a path into two (or more) concurrent segments.

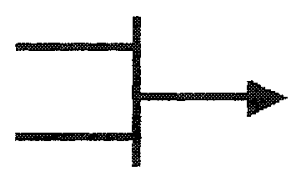

Figure 3.3: (a) AND-join

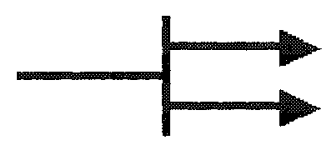

(b) AND-fork

Static stubs

In order to represent complex maps, UCM provides containers (called stubs) for sub-maps (called plug-ins). Stubs are represented as diamonds. There are two kinds of stubs: Static stub (plain diamond in Figure 3.4) and dynamic stub (dashed diamond in Figure 3.5). Static stubs contain only one plug-in, while dynamic may have multiple plug-ins.

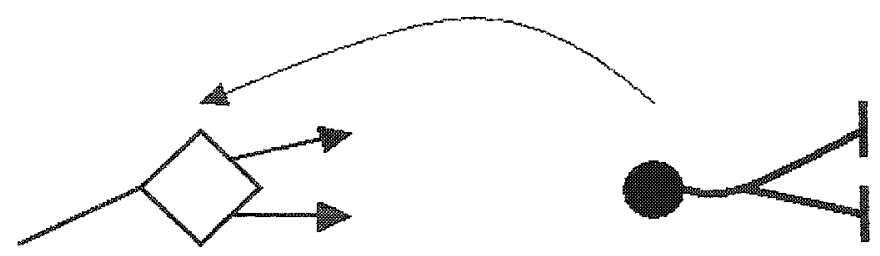

Figure 3.4: Static Stubs have only One Plug-in 
Dynamic stubs (see Figure 3.5)

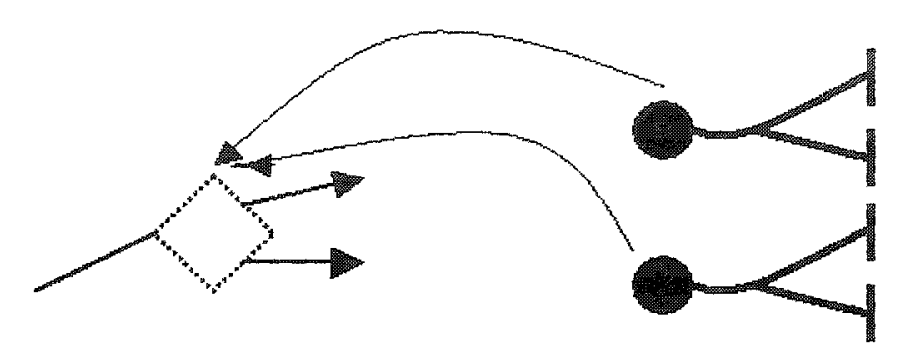

Figure 3.5: Dynamic Stubs may have Multiple Plug-in

As explained previously, UCM is a scenario-based approach for the design of distributed systems. It not only provides systematic and intuitive way to capture functional requirements, but it can be used to analyze the deployment and ownership issue in the Web service domain. This means it can be used to analyze how responsibilities are allocated to components. Other scenario-based approaches, such as Chisel diagram [Aho98], do not offer this function. Furthermore, UCMs provide visual descriptions of behavior at the forest level directly onto diagrams of structure at the same level. Although UML use case diagrams also show existence of and relationships among behavior at the forest level, they do not give visual descriptions of behavior at any level [Buhr98]. That means UCMs are fitter than UML to describe the scenarios. That is why we choose UCM to present the scenarios in the feature interaction detection approach in Chapter 4. The following figure illustrates this. 


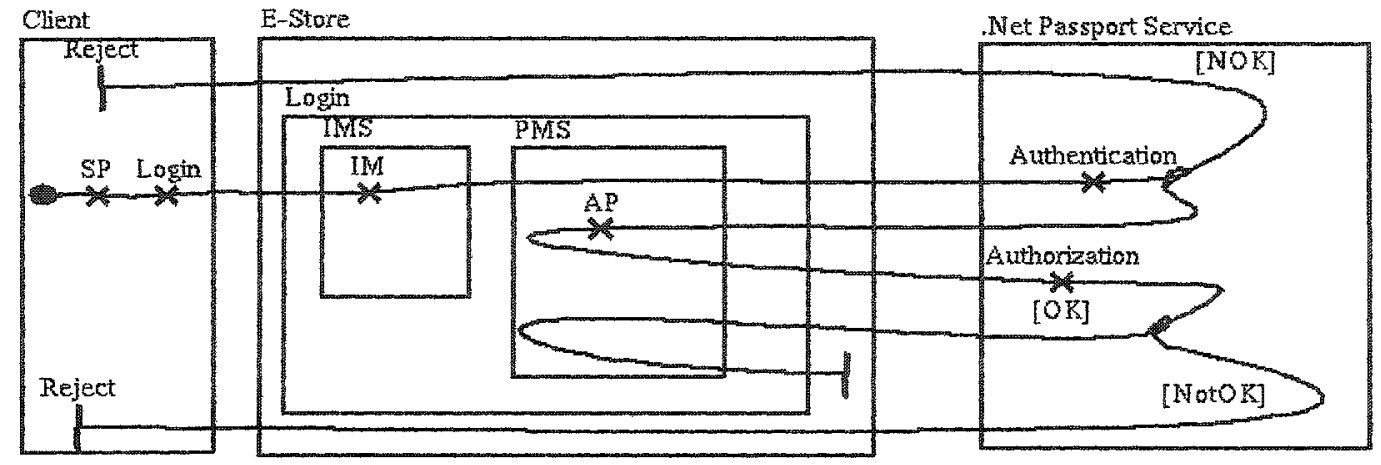

Figure 3.6: Using UCM to Analyze the Deployment and Ownership Issue

Notes for Figure 3.6:

SP-Store Profile; IM - Identity Management; IMS - Identity Management Service; PMS - Profile Management Service; AP-Access Profile

As shown in Figure 3.6, an e-store login function uses two Web services: the Profile Management Service and the Identity Management Service. This improves the system usability. However, if the Identity Management Service is implemented by the .Net Passport Service, it will violate the user privacy because features Authorization and Authentication belong to the same component and owner: .Net Passport Service. More details about this example will be presented in Chapter 5 .

\subsection{Goal-oriented Requirement Language}

\subsubsection{Introduction}

The Goal-oriented Requirement Language (GRL) is used to model and reason about business or system goals and non-functional requirements. It can express various types of concepts during the requirements and high-level architectural design phases. It 
concentrates on exploring why certain choices for behavior and/or structure are made instead of exploring how to make it [Liu01].

It includes three main categories of concepts: intentional elements, links, and actors. The intentional elements are goal, task, soft-goal, beliefs, and resource. They provide answers to questions such as why particular behaviors, informational or structural aspects were chosen to be included in the system requirements.

\subsubsection{GRL Notation Elements}

Goal (see Figure 3.7)

A goal is shown as rounded rectangle. It is a condition or state of affairs in the world that the stakeholders would like to achieve. It could be a business goal or system goal. For example, "Personalization" is one basic goal to be achieved with an e-business system.

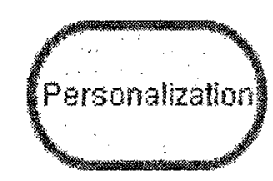

Figure 3.7: Goal Personalization

In this thesis, we use goals to model functional requirements. In Web services, functional requirements are the business or states of the business affairs the individual or organization wishes to achieve. Therefore, it is better to model them using goals instead of soft goals.

Soft-goal (see Figure 3.8) 
A soft-goal also is a condition or state of affairs in the world that the actor would like to achieve. There are no clear-cut criteria for whether the condition is achieved, and it is up to subjective judgment and interpretation of the developer. It is shown as an irregular curvilinear shape. For example, the softgoal "Privacy" should be achieved when we designed an e-business system.

\section{provary}

Figure 3.8: Soft-goal Privacy

In this thesis, we use soft-goals to model non-functional concerns because they are particular to represent non-functional concerns in the future system.

\section{Task (see Figure 3.9)}

A task is shown as a hexagon. It specifies a particular way of doing something. Tasks can also be seen as solutions in the target system, which will satisfy the soft-goals. These solutions provide operations, processes, data representations, structuring, constraints, and agents in the target system to meet the needs stated in the goals and soft-goals. For example, task "Identity Management" is a process to manage the user authentication in an e-business system.

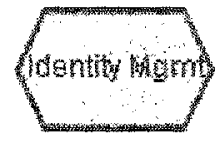

Figure 3.9: Task Identity Management 
In this thesis, we use tasks to model solutions. In Web services, it can specify a particular way of doing something. Furthermore, it also can represent internal Web services. Other elements in GRL model cannot be used to do it.

Actor (see Figure 3.10)

An actor shown as a circle is an active entity that carries out actions to achieve goals. In the Web service domain, it may consist of one or more Web services. It may have a boundary shown as a gray shadow, with intentional elements inside.

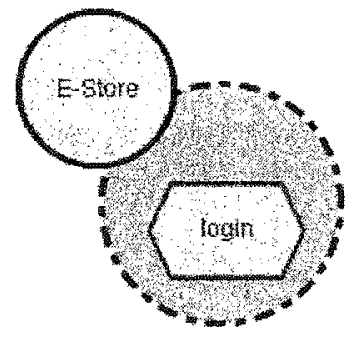

Figure 3.10: An Actor E-Store with a Task Login

We use actors to model external Web services in this thesis. If Web services are internal ones, in other words, if they belong to other Web services, we will not use actors to model them because of the limitation of the tools. Actors are good to model Web services because Web services are also active entities.

Belief (see Figure 3.11)

Beliefs are used to represent design rationale. A belief is shown as an ellipse. In the following example, if the user tries to place an order by two Web services: Local Order Process Service (LOPS) and Remote Order Process 
Service (ROPS). If the local item quantities are less than the user required, LOPS will invoke ROPS to place an order automatically. In this case, the request to ROPS may be redirected to LOPS. This means LOPS will invoke itself.

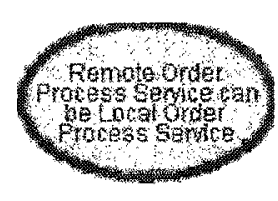

Figure 3.11: Belief Example

\section{Resource (see Figure 3.12)}

A resource is an (physical or informational) entity, with which the main concern is whether it is available. It is shown as rectangles. In an e-business system architecture, network bandwidth is a resource that must be available.

$$
\text { Bandwoth }
$$

Figure 3.12: Resource Bandwidth

\subsubsection{GRL Intentional Links}

Means-ends Relationship (see Figure 3.13)

It is used to describe how goals are in fact achieved. 


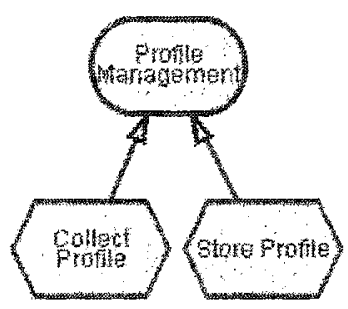

Figure 3.13: Goal Means-ends Structure

In an e-business system, the user profile management includes two operations: collect profile and store profile. As shown in Figure 3.13, in GRL model, a goal Profile Management can be achieved by two tasks Collect Profile and Store Profile.

\section{Decomposition Relationship (see Figure 3.14)}

It is used to define what other sub-elements needs to be achieved or available in order for a task to be performed.

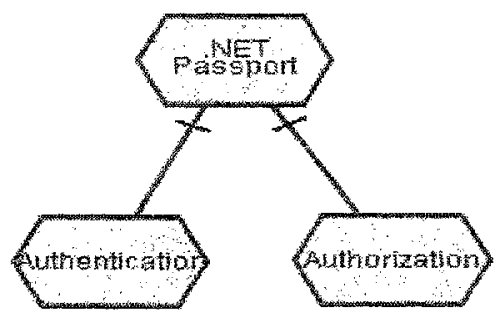

Figure 3.14: Task Decomposition Structure

In an e-business system, Microsoft. NET Passport Service can be used as the user identity management service. This service can authorize and authenticate the user. As shown in Figure 3.14, in GRL model, if task .NET Passport needs to be performed, the sub tasks Authentication and Authorization need to be achieved. 
* Contribution Relationship

It is used to describe how soft-goals, tasks, and links contribute to others.

They could be either positive or negative (see Figure 3.15 [Liu01]), and sufficient/insufficient.

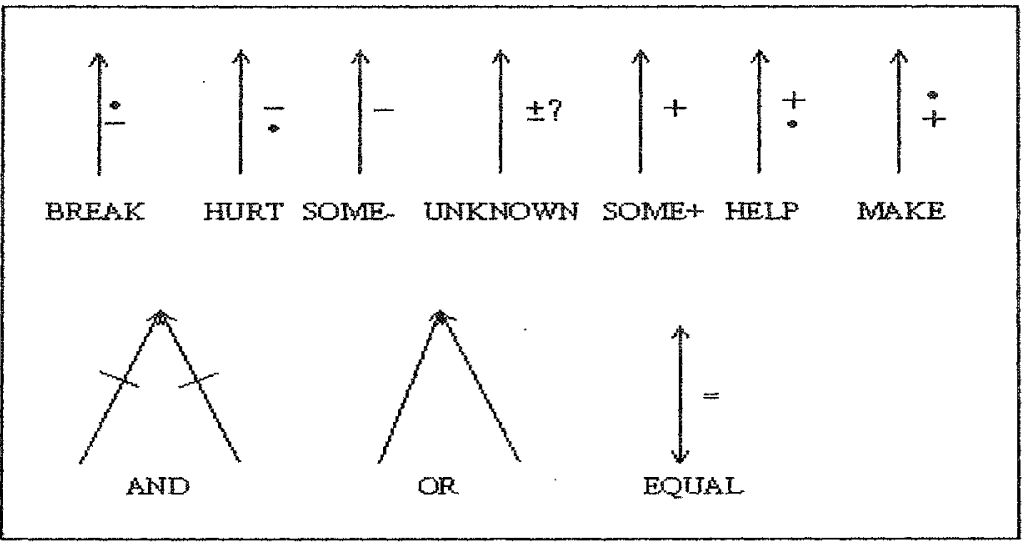

Figure 3.15: Contribution Relationship Graphical Notation

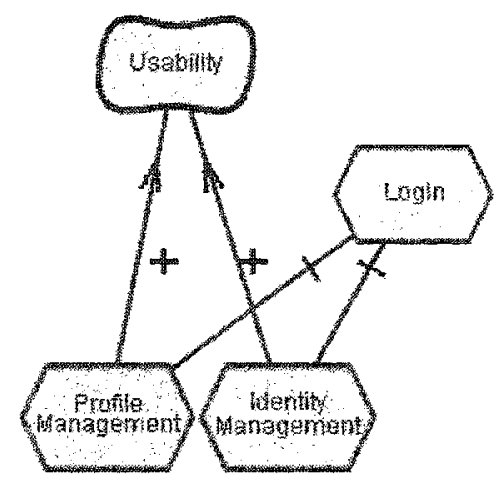

Figure 3.16: Soft-Goal Contribution Structure

As shown in Figure 3.16, in an e-business system, login function includes Profile Management and Identity Management. These two features make the system easy to use; hence, they improve the usability of this system. In GRL 
model, tasks Profile Management and Identity Management contribute to the soft-goal Usability.

In order to simplify the illustration, we will not make use of the different

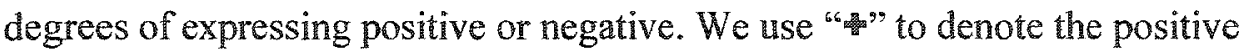
relationship in this thesis, and use " $"{ }^{66}$ to denote the negative relationship.

\section{Correlations Links}

There are used to express knowledge about interactions between intentional elements in different categories, and to encode such knowledge. It is the same as a contribution link except that the contribution is not an explicit desire, but a side effect (see Figure 3.17 [Liu01]).

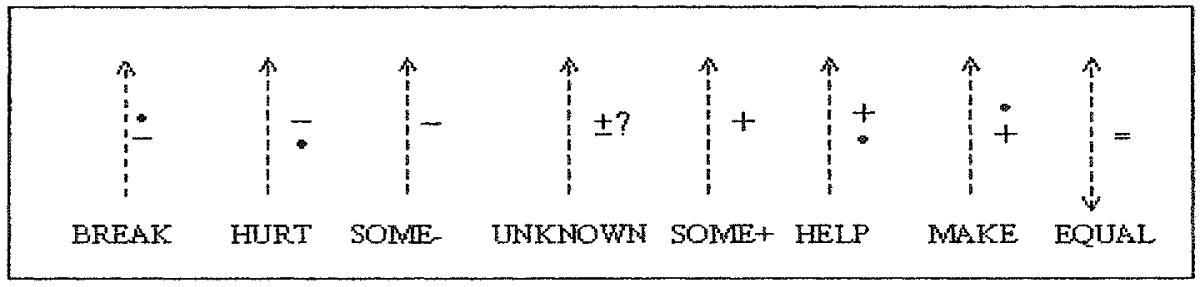

Figure 3.17: Correlations Links Graphical Notation

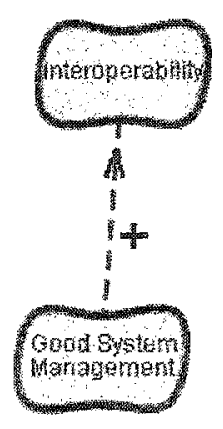

Figure 3.18: Model Structure Series Connected with Correlation 
In an e-business system, good system management will be helpful to the system interoperability. As shown in Figure 3.18, in GRL, soft-goal Good System Management has a correlation to the soft-goal Interoperability.

In order to simplify the illustration, we use "\$" to denote the positive relationship in this thesis, and use " " $"$ to denote the negative relationship.

\section{Dependency}

This statement describes an intentional relationship between two actors. One actor (depender) depends on another actor (dependee) on something (dependum). The dependum can be a goal, a soft-goal, a resource, or a task.

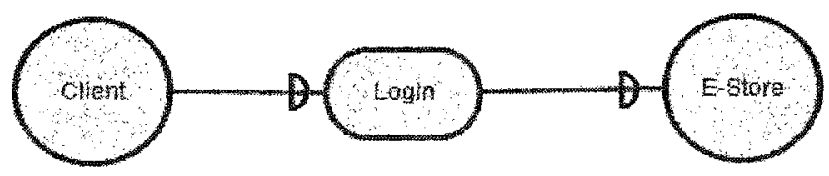

Figure 3.19: Actor Client depends on Actor E-Store on Login As shown in Figure 3.19, in an e-store system, if a client wants to access the e-store system, he/she needs to login this system first. In GRL model, this scenario includes two actors: Client and E-Store. The actor Client depends on the actor E-Store to login the system. The login will be modeled by a goal Login.

As explained previously, the GRL models and reasons about the requirements by goal graphs. Different relationships and requirements can be shown by the goal graphs. In particular, non-functional requirements, such as Usability, Performance, and so forth, can 
be represented in a system. The relationships among them also can be represented and analyzed. The feature interactions among them will be modeled and detected. Eventually, The system design will be refined and optimized by these analyses. Other existing detection approaches are not particular consideration on non-functional concerns. Therefore, the GRL will be useful to detect the interactions among the non-functional concerns. In the approach presented in Chapter 4 , it will be used to model and analyze the non-functional concerns.

\subsubsection{Reasoning about Design Alternatives}

GRL can be used to reason about design alternatives. The following word processing example [W\&E04] illustrates how to do it. This example is used to check the spelling and format the output. It includes three actors: Word Processing Service, Spell Checking Service, and Formatter. As shown in Figure 3.20, the word processing invokes the spellchecking and the formatting. This improves the service's maintainability. The formatting will be performed by a third party service, which also implements a spellchecker function. This spellchecker function may use a different language dictionary with the Spell Checking Service in Word Processing Service, and this will hurt the correctness of this service. We use correlation instead of contribution to model this relationship because it is a side effect occurred in different components. 


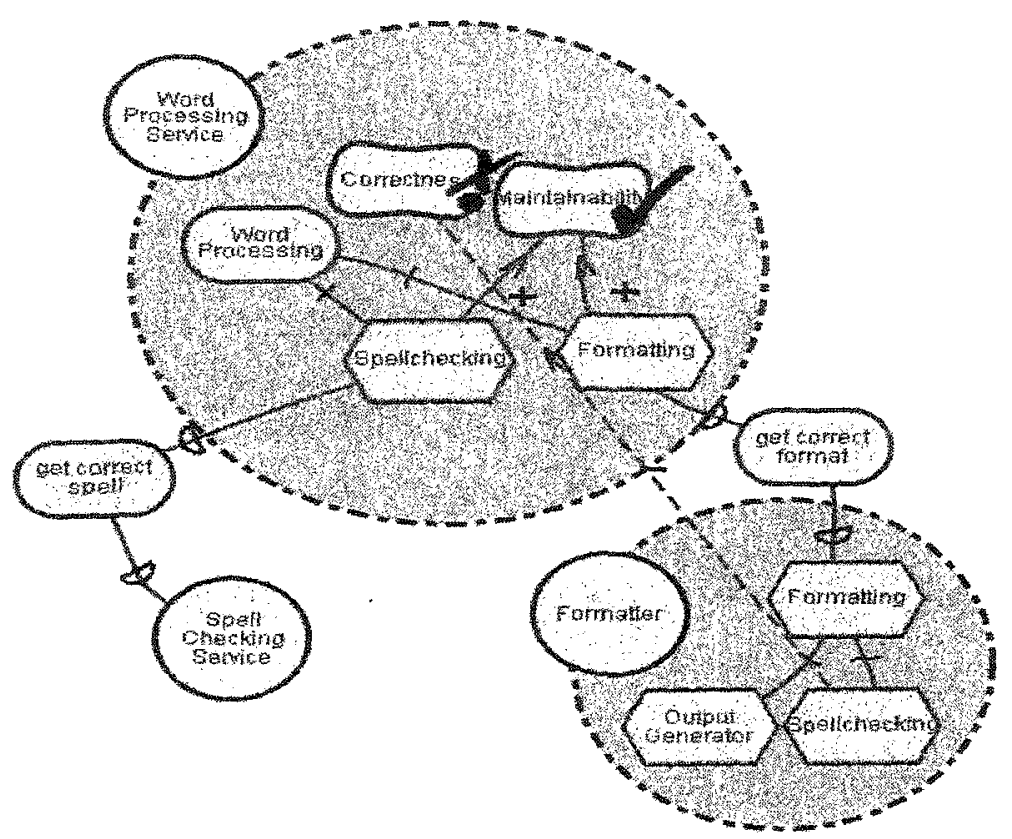

Figure 3.20: Goal Conflicts in the Word Processing Example

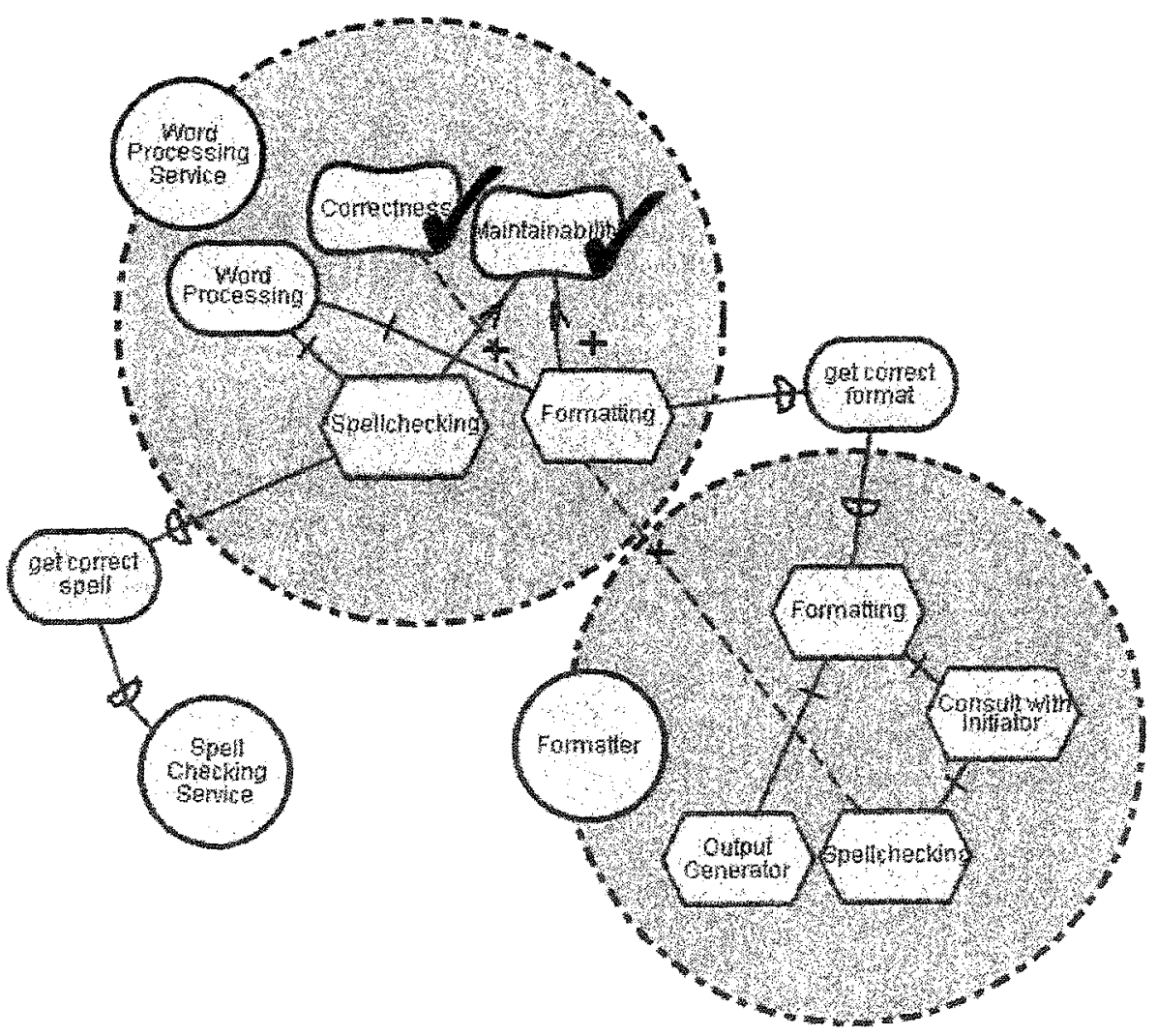

Figure 3.21: Consult with Initiator before Invoking the Spellchecking 
As the above explained, the reason for this interaction is that two spellchecking services may use different dictionaries. We need to consider design alternatives. We could consult with the initiator before invoking the spellchecking (see Figure 3.21). This change would avoid the goal conflict.

\subsection{Chapter Summary}

In this chapter we have introduced several concepts involved in the use of the UCM and GRL framework, such as OR-forks/joins, AND-forks/joins, dynamic stubs, goal, task, soft-goal, belief, relationship, and so forth. We also reviewed parts of their notations, which will be used in this thesis.

As we presented in the previous sections, the UCM and GRL framework provide ample features for modeling several design alternatives. As system designers or analysts, we want to benefit from those features and take advantage of good results from previous efforts. We will use them for detecting feature interaction problems in the Web service domain at the requirements stage because UCM can be used to analyze scenarios and to reason about components and their relationships, while GRL can be used to analyze nonfunctional requirements in systems. We will present this topic in the next chapter. 


\section{Chapter 4 Feature Interaction Categories and a Detection Approach}

\subsection{Three Dimensions for Categories in Web Services}

In the telecommunication domain, Cameron et al. [Cameron94] presented a classification of feature interactions to show the scope of the problem and to analyze emerging solutions. They discussed two different ways of categorizing feature interactions in their classification: by the nature of interactions and by the cause of interactions. In the Web service domain, feature interactions have some new complications, due to the distributed nature of Web services themselves. For example, third party Web services can be used in the system. Hence, it is necessary to present a different classification of feature interactions in the Web service domain that extends the classification in the telecommunication domain. In this thesis, an initial classification will be presented. Further development will be expected in the future. This classification includes three dimensions of categorization of the nature of interactions: type, scope, and role. Type includes Functional and Non-Functional. Scope includes Internal and External. Role includes Single Role and Multiple Roles. It is specific to the Web service domain because its value relies on three roles of Web services. We will also categorize the interaction by their causes, such as Violation of Assumptions about Service (see \$4.2.1), Different Invocation Order (see \$4.2.2), 
Resource Contention (see \$4.2.3), Policy Conflict (see \$4.2.4), Goal Conflict (see

\$4.2.5), Deployment and Ownership (see \$4.2.6), and Encapsulation and Information

Hiding (see \$4.2.7). In particular, last three causes are specific to the Web service domain.

\subsubsection{Type}

In general, irrespective of domains, we can consider two categories in this dimension: functional and non-functional. The differences and definitions of those two categories depend on the application system. Usually, people pay more attention to study functional interactions than non-functional ones. However, the fact is, each functional composition will impact the satisfaction of non-functional concerns, such as usability, performance, privacy, and security. Thus, it makes sense to discuss non-functional interactions as well as functional interactions in the Web service domain.

Definition 1 (Functional Interaction). Functional interactions are some way in which a feature or features (functionality) modify or influence another feature in defining overall system behavior. Like in the telecommunication domain, feature interactions are inevitable in the Web service domain, as a completely independent feature can accomplish so little. Web services will need to use other features in other Web services. For example, a word-processing feature in the word processing service could use the spell checking feature in a third party spell checking service and a formatting feature in a formatting service. Undesirable feature interactions may occur during the composition. These may cause Web services to fail or break the whole application system. 
Definition 2 (Non-Functional Interaction). We consider non-functional interaction based on relative functional scenarios. In this thesis, we do not call them interactions unless the relationship among non-functional concerns causes conflict. Furthermore, all those non-functional requirements need to be concerned by the related actors. In other words, non-functional concerns in the feature interaction need to affect the total satisfaction of the actors. If the actors do not care about one of them, we do not call the conflict among them an interaction.

\subsubsection{Scope}

This dimension distinguishes internal feature interactions from external feature interactions according to the scope of an actor. As we introduced in Chapter 3, an actor is an active entity that carries out actions to achieve goals. It may include a single Web service or multiple Web services. Internal feature interactions arise when feature conflicts occur inside an actor, while external feature interactions occur when feature conflicts occur between two actors. We distinguish feature interactions by this dimension because the detecting methods and solutions in the Web service domain may vary with the different scope of feature interactions.

Definition 3 (Internal Feature). Internal features are features that are defined or implemented inside an actor.

Definition 4 (Internal Feature Interaction). Internal feature interactions are feature interactions that occur between two internal features of a same actor (see Figure 4.1). For 
example, as shown in Figure 4.1, the features Transaction Management and Logging belong to an actor: Payment Service. The feature Transaction Management is used to manage the long-running transaction. The feature Logging is used to log the payment information into the $\log$ file during the transaction. The nature of transaction does not allow that any information is sent out before the transaction finished. However, the nature of Logging is that messages can be sent without any limitation. The feature interaction between them (shown by symbol) is an internal one. More details of this feature interaction will be given in Example 5.5.

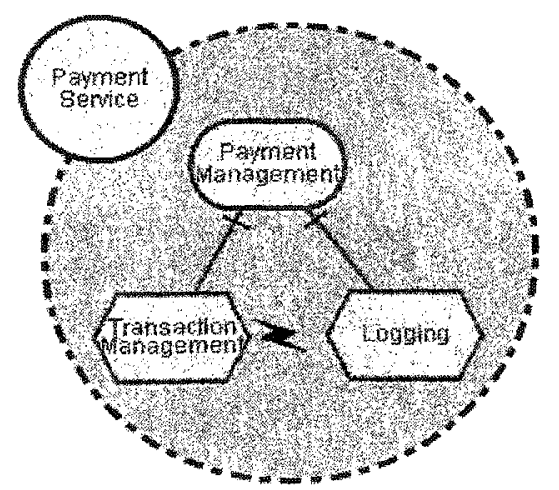

Figure 4.1: An Internal Feature Interaction between Features Transaction Management and Logging

Definition 5 (External Feature). External features are features that are defined or implemented outside an actor.

Definition 6 (extermal Feature Interaction). External feature interactions are feature interactions that occur between features in two actors. In internal feature interactions, the features exist in an actor, who can control these features inside it. External features exist in different actors. The actors cannot control the other actor's features. For example, as 
shown in Figure 4.2, Boogle is an actor, which includes Boogle Search Service (BSS).

$O^{\prime}$ Rally is another actor, which includes O'Rally Book Access Service (BAS). BSS

provides the feature Search Request Cache. BAS provides the feature Book Search. The feature Search Request Cache is used to cache the request links. If request link exists in the Boogle cache, BSS will not redirect the request to the Book Search of $B A S$ because it assumes that the required book information has existed in the cache. The feature Book Search of BAS will never store the contents of the books in the cache of the client. When a user requests book information from $B A S$ at the first time, he will get the right result. If he requests the same book information again, he will not get the right result because his request link has been saved in the cache after the first request. When he requests again, the feature Search Request Cache will not redirect his request to the $B A S$. However, Book Search of $B A S$ never saves the book information at the client cache. This is a conflict between the features Search Request Cache and Book Search. The feature interaction between them is an external one. More details will be given in Example 4.4.

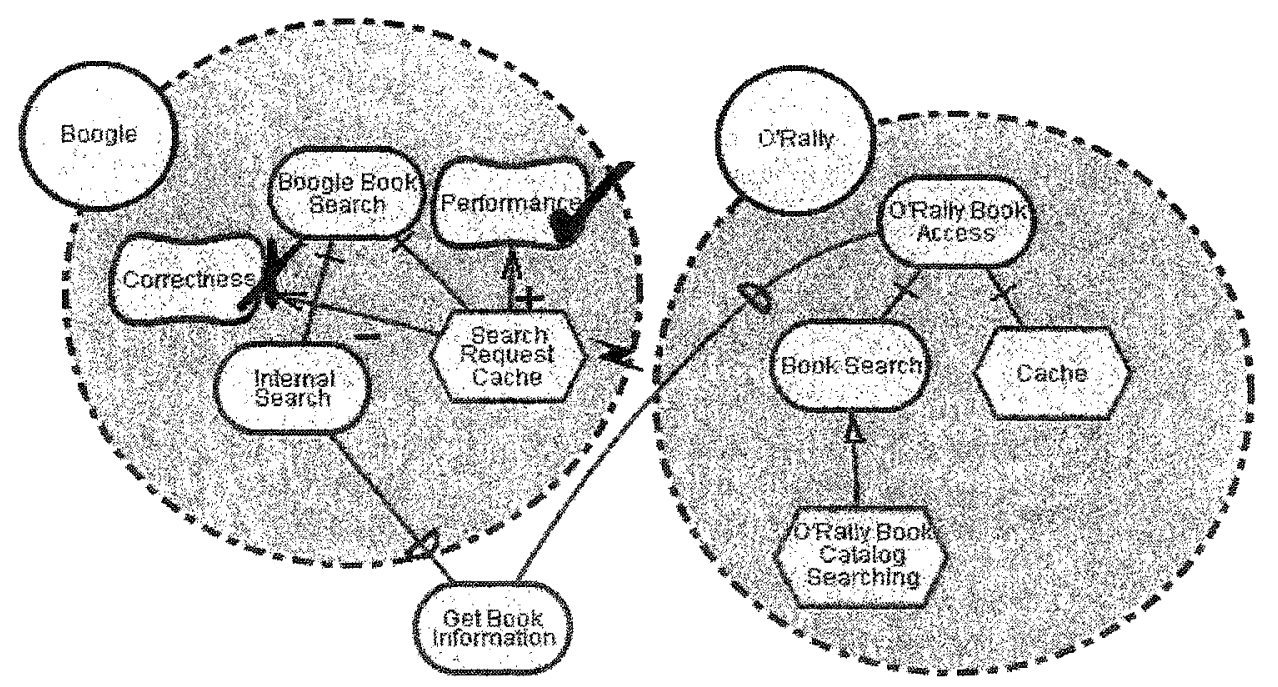

Tigure 4.2: An External Interaction between Features Search Request Cache and Book Search 


\subsubsection{Role}

As explained previously, there are three roles in the Web service architecture: consumer, provider, and broker. Feature interactions may occur with the different number of roles an actor plays. This dimension defines two kinds of feature interactions based on the roles: Single Role (SR) and Multiple Roles (MR). If consumer knows the address of the provider, it can connect to provider directly without broker. In this thesis, in order to simplify the analysis of the examples, we will assume all service consumers can connect to the service providers directly. Therefore, we do not consider broker.

Definition 7 ( $S R$ Feature Interaction). $S R$ feature interactions are feature interactions that occur between one or more actors, in which all of them act in a single role.

Definition 8 ( $M R$ Feature Interaction). $M R$ feature interactions are feature interactions that occur between one or more actors, in which at least one of them acts in multiple roles.

$M R$ feature interactions are more complex than $S R$ ones because actors in $M R$ feature interactions will need to take more responsibilities than in $S R$ ones. In SR feature interactions, actors either provide or consume the service. However, in MR ones, actors need to do both. This additional responsibility of actors increases the complexity of feature interactions. 
$M R$ feature interactions occur easier than $S R$ ones because of the more responsibilities in actors of $M R$ feature interactions. In general, more responsibilities increase the possibility of the occurrence of the feature interactions.

$M R$ feature interactions are more difficult to detect and resolve than $S R$ ones because the relationships among the features involved in MR feature interactions are more complex than in SR feature interactions. Therefore, the detection methods and the solutions for MR feature interactions are more complex than SR feature interactions.

\subsection{Categorization of the Cause of Interaction}

\subsubsection{Violation of Assumptions about Services}

When we develop a Web service, we need to make some assumptions about how to consume this Web service. During the Web service composition, violation of those assumptions may result in some feature interactions. Semantic mismatch is also one of the violations of assumptions.

Example 4.1, Example 4.2, and Example 4.4 are examples to support this cause.

\subsubsection{Different Invocation Order}

During the static Web service composition, different application systems with different concerns may have different composition (or invocation) orders. Not all composition orders can result in expected results. For example, the effect of some orders may modify the ultimate goal of Web services while others may break the total satisfaction of Web services. Irrespective of those results, feature interactions occur. 
Example 4.3 is an example to support this cause.

\subsubsection{Resource Contention}

Resource contention will degrade Web service performance or break the Web service application system. The resources include network bandwidth, disk space, host computer CPU, main memory, secondary memory, and $\mathrm{I} / \mathrm{O}$ channels.

Example 5.4 in Chapter 5 is an example to support this cause.

\subsubsection{Policy Conflict}

Policies in Web services are kinds of declarative assertions. In the examples of this thesis, they are used to advertise non-functional capabilities, and provide middleware enhancements to match, interpret, and mediate QoS (Quality of Service) requirements of service providers and service consumers at deployment time and/or runtime.

The current approaches for QoS feature management, such as the use of containers and generative programming [Roman01], AOP (aspect-oriented programming) [Duclos02][Wohlstadter03], or software wrappers [Fraser99], are limited to support Web services QoS management for two reasons [Wohlstadter04]. The first one is those approaches are not truly dynamic. It means that components only implement a functional interface; QoS features are left unresolved until deployment time. Another reason is those approaches are server-centric, and do not consider the issue of matching client-side QoS features to the deployment policy on the server.

Example 5.5 in Chapter 5 is an example to support this cause. 


\subsubsection{Goal Conflict}

In the GRL model, a goal is an objective or a concern. It is used to discover and evaluate functional and non-functional requirements. Goal conflicts [W \&E04] can be considered as results of unanticipated side effects of a solution for achieving one soft goal, such as performance. All examples except Example 5.6 in Chapter 5 illustrate this.

\subsubsection{Deployment and Ownership}

This cause has been studied in [W\&E04]. The intrinsically open and distributed nature of Web services is the source of this problem. Deployment discusses where a service or a feature should be deployed. Ownership means who hosts such services. Different deployment and ownership decisions can lead to different solutions. The same owner with ownership over multiple services can lead to conflicts of interest or goals.

Example 4.5 and Example 5.1 in Chapter 5 illustrate this cause.

\subsubsection{Encapsulation and Information Hiding}

In an object-oriented approach, Encapsulation and Information Hiding are used to hide a lot of detail between objects. Objects are responsible for hiding private data from the outside user. 
The component-based approach raises the granularity of the object-oriented approach. In comparison with an object-oriented approach, this approach allows those components to be invoked remotely, as the cost of operation invocation becomes infeasible high.

Web services approach have a big granularity because of their loosely coupled architecture, which allows individual nodes in a distributed system to change without affecting or requiring change in any other part of the system. In comparison with objectoriented and component-based approaches, objects and components can be hidden inside a Web service. Furthermore, the communication across company boundaries can be easily implemented by this approach. Because of its larger granularity, it is possible that more dependencies and assumptions are hidden in the services. Therefore, the feature interaction can also occur easily.

Example 4.1 and Example 4.2 are examples to support this cause.

\subsection{Classification}

As explained previously, those three dimensions present different views of the feature interaction problem. Combining multiple dimensions may help us to make an accurate categorization. In the following section, we will present eight categories of feature interactions (see Table 4.1 ) by the nature. The related examples to verify these categories will be presented in the following e-book access system and the case study in Chapter 5 . The e-book access system mainly concentrates on illustrating the functional interactions, while non-functional interactions will be illustrated in Chapter 5. 


\begin{tabular}{|l|l|l|}
\hline Examples & $\begin{array}{l}\text { Acronyms of the } \\
\text { Categories }\end{array}$ & Fame of the Categories \\
\hline E4-1 & FIMR & $\begin{array}{l}\text { Functional-Internal- } \\
\text { SingleRole }\end{array}$ \\
\hline E4-2, E4-3 & $\begin{array}{l}\text { Functional-Internal- } \\
\text { MultipleRoles }\end{array}$ \\
\hline E5-6 & FEMR & $\begin{array}{l}\text { Functional-External- } \\
\text { SingleRole }\end{array}$ \\
\hline E5-5 & NFISR & $\begin{array}{l}\text { Functional-Extemal- } \\
\text { MultipleRoles }\end{array}$ \\
\hline E5-2, E5-3, E5-4 & NFIMR & $\begin{array}{l}\text { Non-Functional-Internal- } \\
\text { SingleRole }\end{array}$ \\
\hline E5-7 & NFESR & $\begin{array}{l}\text { Non-Functional-Internal- } \\
\text { MultipleRoles }\end{array}$ \\
\hline E5-1, E5-8 & NFEMR & $\begin{array}{l}\text { Non-Functional-External- } \\
\text { SingleRole }\end{array}$ \\
\hline
\end{tabular}

Table 4.1: Eight Categories of the Feature Interactions in Web services

\section{Notes for Table 4.1:}

\section{Categories:}

FISR-Functional-Internal-SingleRole Feature Interactions FIMR - Functional-Internal-MultipleRoles Feature Interactions FESR-Functional-External-SingleRole Feature Interactions FEMR-Functional-External-MultipleRoles Feature Interactions NFISR - Non-Functional-Internal-SingleRole Feature Interactions NFIMR - Non-Functional-Internal-MultipleRoles Feature Interactions NFESR-Non-Functional-External-SingleRole Feature Interactions NFEMR - Non-Functional-External-MultipleRoles Feature Interactions

2. Examples:

E4-1-Example 4.1; E4-2-Example 4.2; E4-3-Example 4.3; E4-4-Example 4.4; E4-5-Example 4.5; E5-1-Example 5.1; E5-2-Example 5.2; E5-3-Example 5.3; E5-4-Example 5.4; E5-5-Example 5.5; E5-6-Example 5.6; E5-7- Example 5.7 E5-8-Example 5.8

This e-book access system includes several fictitious examples to quickly illustrate categories. It provides an online searching and accessing function to the Satori publication catalog of technical books and their information to online subscribers. It is a Web application that makes use of six Web services. The following architecture depicts its functionalities. 
As shown in Figure 4.3, the ovals displayed in light gray are Web services. They are Local Book Search Service, Boogle Book Search Service, Satori Book Service, O'Rally Book Catalog Service, O'Rally Book Rating Service, and Payment Service. These Web services will be composed to perform the functionalities of the system.

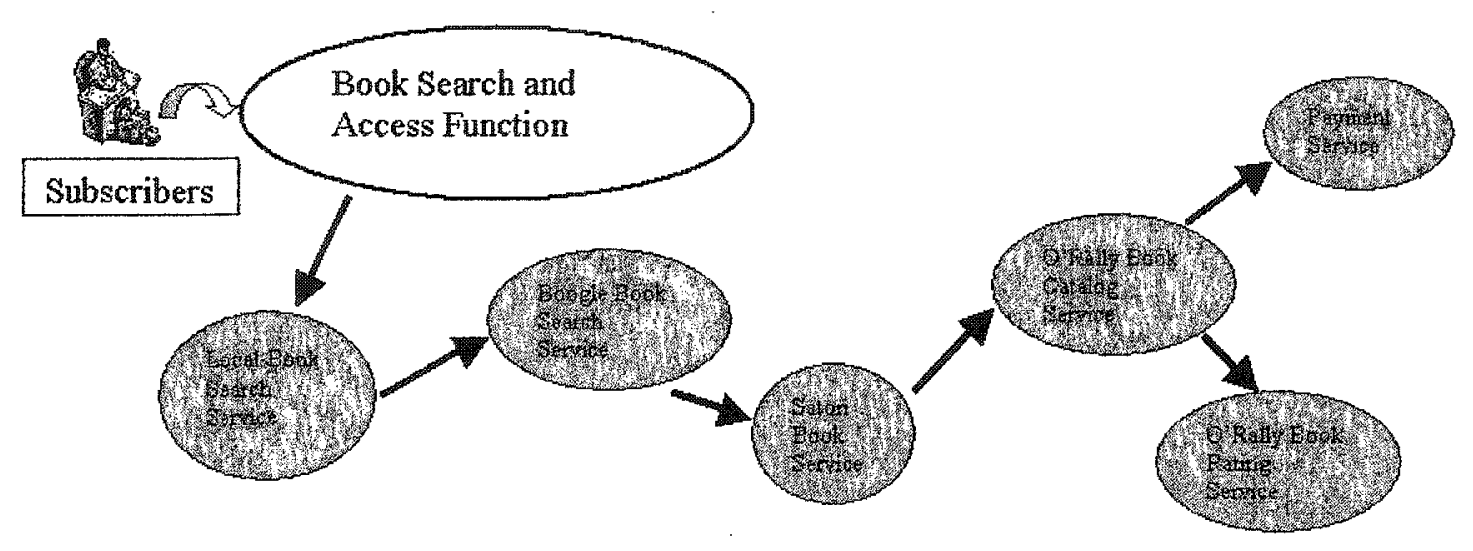

Figure 4.3: The Architecture of the E-Book Access System

The following GRL model depicts the functional requirements of this system by visualizing dependencies relations that exist among actors and their alternatives. 


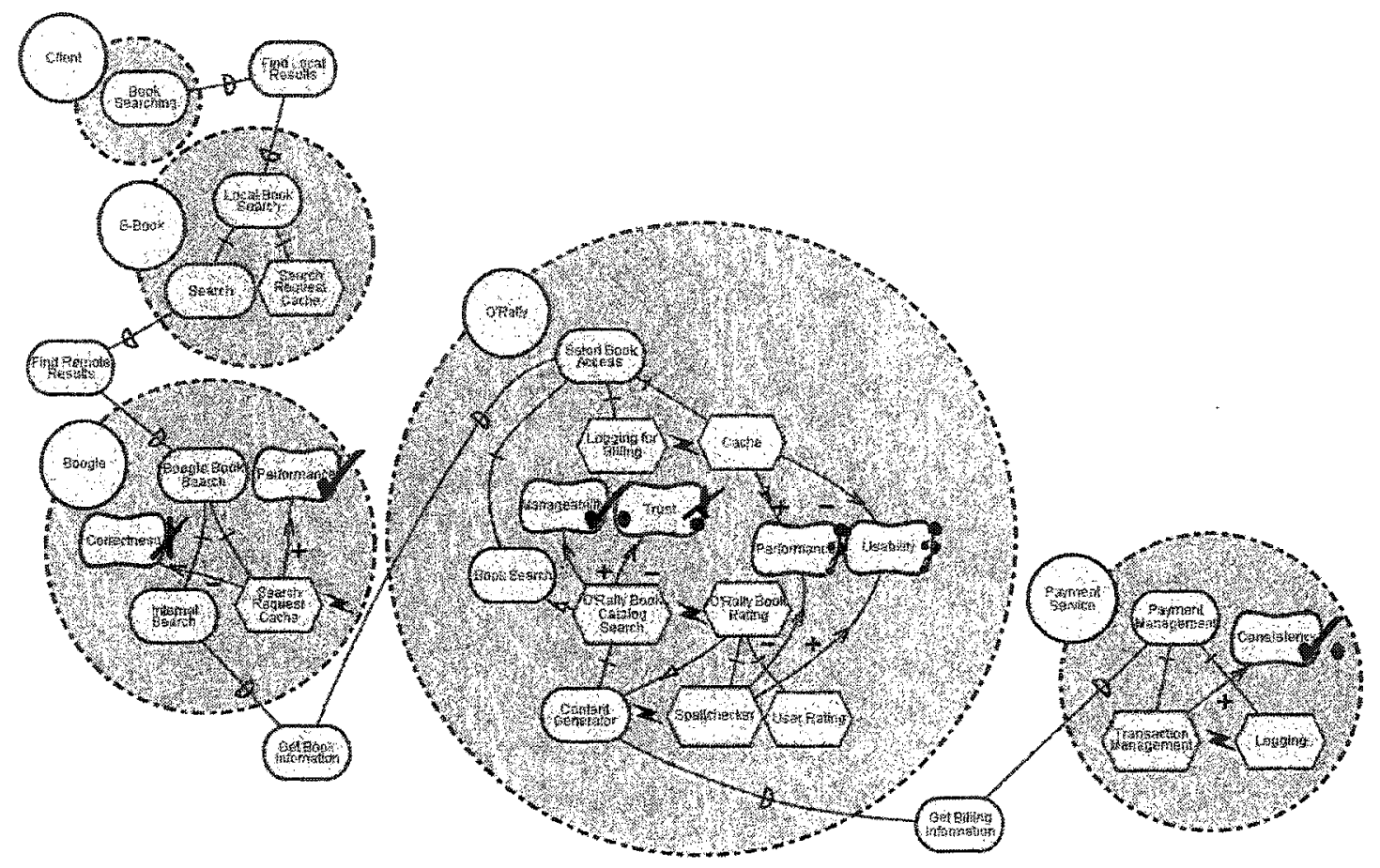

Figure 4.4: The GRL Model for the E-Book Access System

As shown in Figure 4.4, there are five actors shown as circles: Client, E-Book, Boogle, O'Rally, and Payment Service, which act as the active entities that carry out actions to achieve goals. The intentional relationships among them are dependencies. For example, the actor Client depends on the actor $E$-Book on finding local results. Several goals and their features also have been presented in this model. There are several feature interactions among those features or goals as shown by symbol. They will be covered in Examples 4.1 4.5 in this chapter. The rest of this section will introduce six Web services and their features.

1) Local Book Search Service: 
There are two features in this service: Search and Search Request Cache. Search feature redirects the search link to the Boogle Book Search Service. Search Request Cache feature is used to cache the search link.

- Search Feature:

Search feature redirects the client request to the Boogle Book Search Service.

2) Boogle Book Search Service:

Boogle is a search engine. Book publishers can send their books to Boogle, where they will be scanned and added to Boogle's search index for free. This service lets users search the contents of these books and redirects the requested links to a book catalog service called Satori Book Service if no result can be found. There are two features in this service: Internal Search and Search Request Cache.

- Internal Search Feature:

This feature is used to search the contents of the books. If no result can be found, it will check whether requested links exist in the cache. If requested links do not exist in the cache, this feature will redirect the requests to Satori Book Service.

- Search Request Cache Feature:

This feature saves requested links in the cache.

\section{3) Satori Book Service:}

This service is provided by O'Rally. It provides access to the Satori publication catalog of technical books. It allows users to select books from the catalog for their personal bookshelf. Users can view the entire contents of these books. The content of the 
books is not stored on the user's local computer, but rather it is dynamically delivered via the Internet. It also provides a logging feature to record the user's requests. Those records are used for billing. There are three features in this service: Book Search, Cache, and Logging for Billing.

- Book Search Feature:

If requested links do not exist in the cache, this feature will invoke the O'Rally Book Catalog Service to get the contents of the book, and return the result to the client.

- Cache Feature:

This feature saves the requested links and the contents of the book in the cache.

- Logging for Billing Feature:

This function writes the user's access information into a log file when a user accesses the book catalog. The information will be used for billing purpose.

4) O'Rally Book Catalog Service:

This service is used to search the Satori catalog and create the contents of book for users, which includes user comments and user rating of books. It consists of several features, such as ContentGenerator and Content Looking/Indexing. In this thesis, we just use the feature ContentGenerator to illustrate feature interaction.

- ContentGenerator Feature:

It is used to generate the required contents of the book, user comments, and user rating information. 


\section{5) O'Rally Book Rating Service}

This service rates the book before the contents of the book have been generated. The contents of the book include user comments, which will be checked by a spellchecker. There are two features in this service: Spellchecker and User Rating.

\section{- Spellchecker Feature}

This feature checks the spelling of user comments. This feature is implemented by a third party service. It is used for the user comments checking.

- User Rating Feature

This feature rates books by users.

\section{6) Payment Service}

This service provides online payment management. It consists of two features:

Transaction Management and Logging.

- Transaction Management Feature

This feature manages the payment transaction. If payment is successful, it will allow the ContentGenerator Feature of the O'Rally Book Catalog Service to generate contents for user. Otherwise, it will rollback the transaction. This feature assumes the payment process information should not be sent until transaction commits.

- Logging Feature

This feature logs the payment messages to a log file. This feature assumes that logs can be sent independently without any limitation. 
As explained above, Examples $4.1 \sim 4.5$ present five interactions among those features. Example 4.1 illustrates the category FISR. Example 4.2 and Example 4.3 are for FIMR. Example 4.4 is for FEMR. Example 4.5 is for NFIMR. Other categories will be illustrated by the case study in Chapter 5 .

\subsubsection{FISR (Functional-Internal-SingleRole Feature}

\section{Interaction)}

Definition 9 (FISR Feature Interaction). FISR feature interactions are feature interactions that occur among functional requirements within a single actor. This actor acts in a single role. This is the simplest feature interaction category of eight categories in Web services because of three reasons. First, functional interactions have been better studied and have more tool supports than non-functional interactions. Therefore, the scenario of functional interactions can be modeled easier than non-functional ones by the existing tools, such as UCM, and LOTOS. The functional interactions can be detected and resolved easier than non-functional ones. Second, if feature interactions occur in a single actor, this actor has a full control for all features involved in the feature interactions. It is easier to detect and resolve the feature interactions in a single actor than in multiple actors. The last reason is that one actor acting in a single role will have less concerns of side effects than acting in multiple roles. The following Example 4.1 illustrates this category. 
Example 4.1: Transaction Management vs. Logging

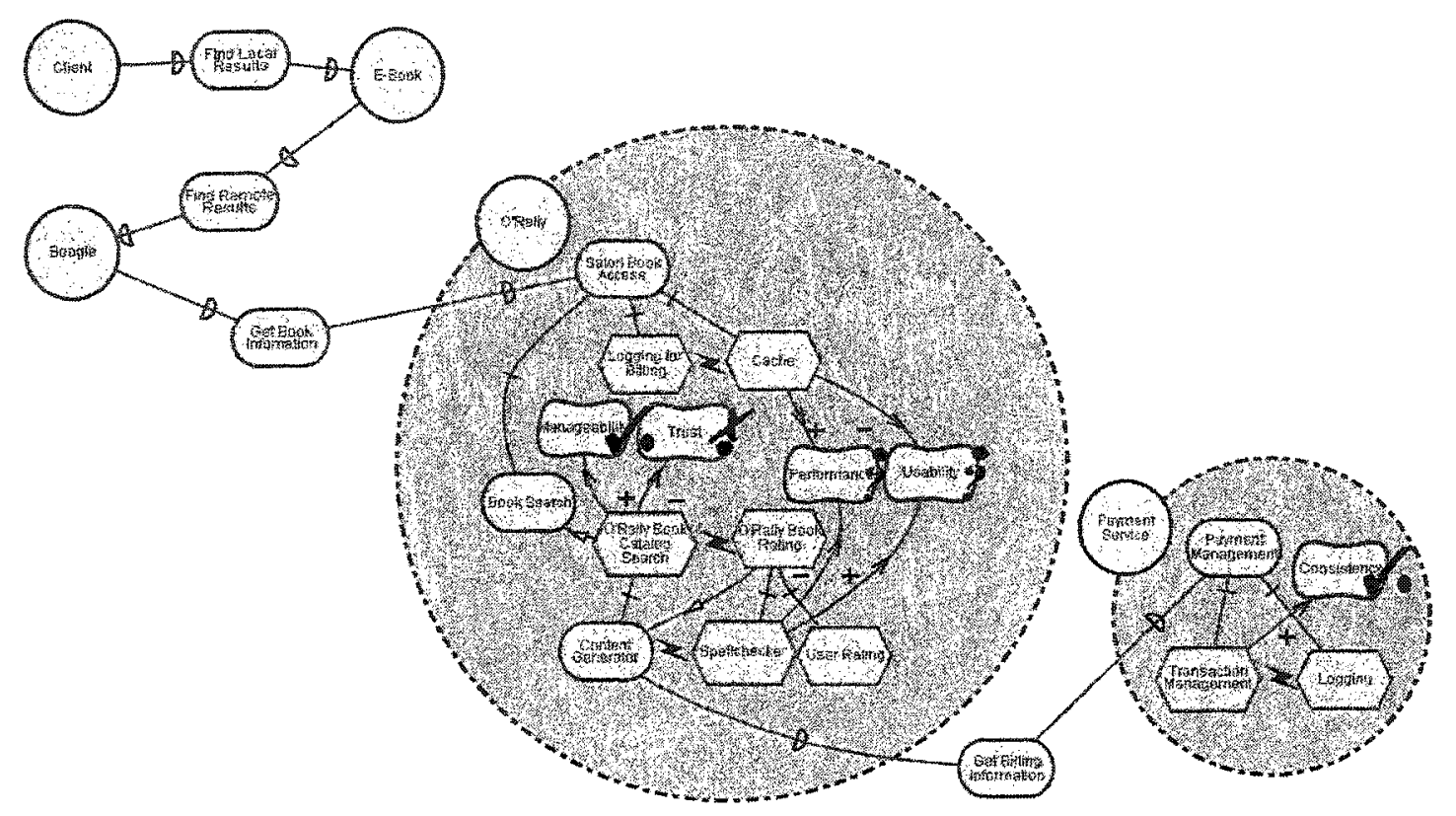

Figure 4.5: The GRL Model for an Interaction between Transaction Management and

\section{Logging}

As shown in Figure 4.5, before the ContentGenerator feature of the O'Rally Book

Catalog Service generates the contents of the books for users, it needs to check whether users have paid for the contents. In order to perform this process, the ContentGenerator feature needs to invoke the Transaction Management feature of the Payment Service. The Transaction Management feature will create a new transaction to handle the whole payment process. If the payment is successful, this feature will allow the ContentGenerator feature to generate the contents. Otherwise, it will roll back the payment transaction. The Payment Service also uses a Logging feature to send the payment process information to a log file in order to track the process. Therefore, even if the transaction is rolled back, the payment process information will also be written into a $\log$ file. In this scenario, to allow a transaction manager to preserve atomicity and 
serializability, the payment process information should not generally be sent until the transaction commits. A logging capability could easily send logs independently, thus conflicting with the ability of the transaction management to satisfy its requirements.

Discussion: In this example, interaction occurs between functional requirements of Payment Service. The feature interaction occurs in a single actor: Payment Service. This actor provides the service to O'Rally Book Catalog Service. However, it does not consume any other services. Therefore, it acts in a single role: Service provider. Hence, it is a FISR interaction. The Transaction Management feature improves this service consistency.

In this example, feature Transaction Management assumes the payment process information should not be sent until transaction commits. However, feature Logging assumes that logs can be sent independently without any limitation. When these two features are implemented in a service, they will conflict because of the violation of assumptions each other. Therefore, the cause of this feature interaction is the Violation of Assumptions about Service (see $\$ 4.2 .1)$.

\subsubsection{FIMR (Functional-Internal-MultipleRoles Feature Interaction)}

Definition 10 (FIMR Feature Interaction). FIMR feature interactions are feature interactions that occur among functional requirements in an actor. This actor acts in multiple roles. It includes two situations. If this actor just includes one Web service, this 
service will consume services in different actors, and it will be called by other services in different actors. If this actor includes more than one Web service, then at least one Web service will invoke other services in different actors, and at least one Web service will be invoked by other services in different actors. It is more complex than FISR feature interactions because the actor takes more responsibilities. More responsibilities mean more invocations among the features. More invocations increase the possibility of the occurrence of feature interactions. The following Examples 4.2 4.3 illustrate this category,

Example 4.2: Logging for Billing vs. Cache

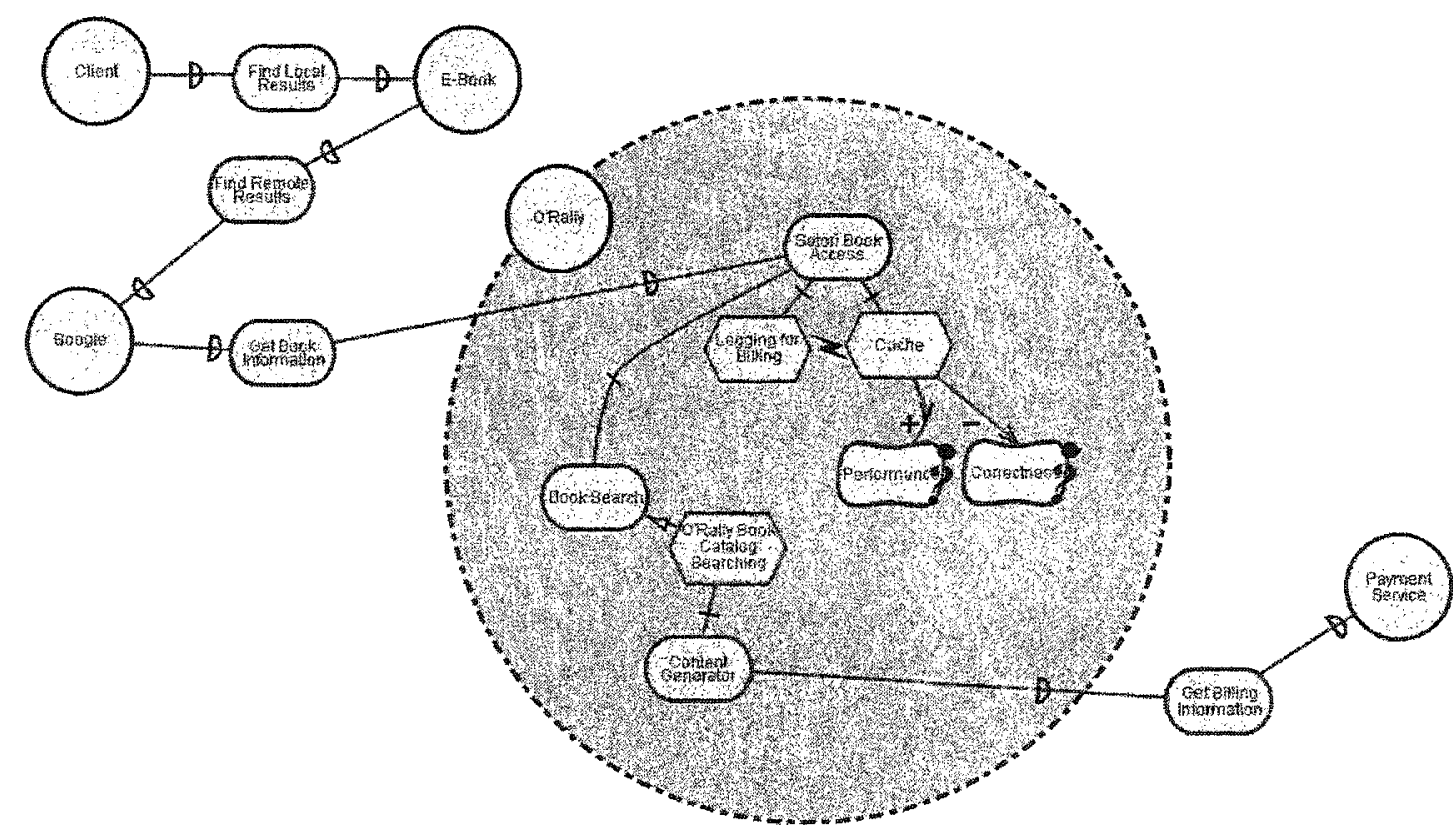

Figure 4.6: The GRL Model for an Interaction between Logging for Billing and Cache

As shown in Figure 4.6, the Logging for Billing feature of Satori Book Service is used to create the book access logs for billing purpose. This feature only works when the users access the catalog. The Cache feature is used to cache the user requested links and the contents of the book. When a user browses the contents of a book for the first time, the 
user needs to access the catalog. Therefore, the Logging for Billing feature will record the requested link in a log file and the Cache feature will cache the requested link and the contents of the book in the cache. When this user browses the same contents of the book a second time, the Cache feature will check the user requested link, if the requested link exists in the cache, it will send the saved contents to the user from its cache instead of accessing the catalog again. Because the catalog has not been searched at this time, the user access logs will not be recorded by the Logging for Billing feature. That means the user will not be charged at this time. The nature of the Logging for Billing feature is to record all access logs of the user, which includes duplicate ones. Although the Cache feature improves the performance, it defeats the purpose of the Logging for Billing feature.

Discussion: The interaction occurs between two functional requirements within an actor: $O^{\prime}$ Rally. Therefore, it is a functional and internal interaction. The actor provides service to Boogle Book Search Service, and it invokes Payment Service. Therefore, the actor acts in two roles: service provider and service consumer. Hence, this is a FIMR feature interaction. Although the Cache feature improves the performance, it results in the incorrect logs for the user. Hence, it hurts the correctness of the Satori Book Service.

The Logging for Billing feature does not know the Cache feature will save the contents for reuse purpose. The Cache feature violates the Logging for Billing feature's assumption. Therefore, Violation of Assumptions about Service (see \$4.2.1) causes this feature interaction. 
Example 4.3: ContentGenerator vs. Spellchecker

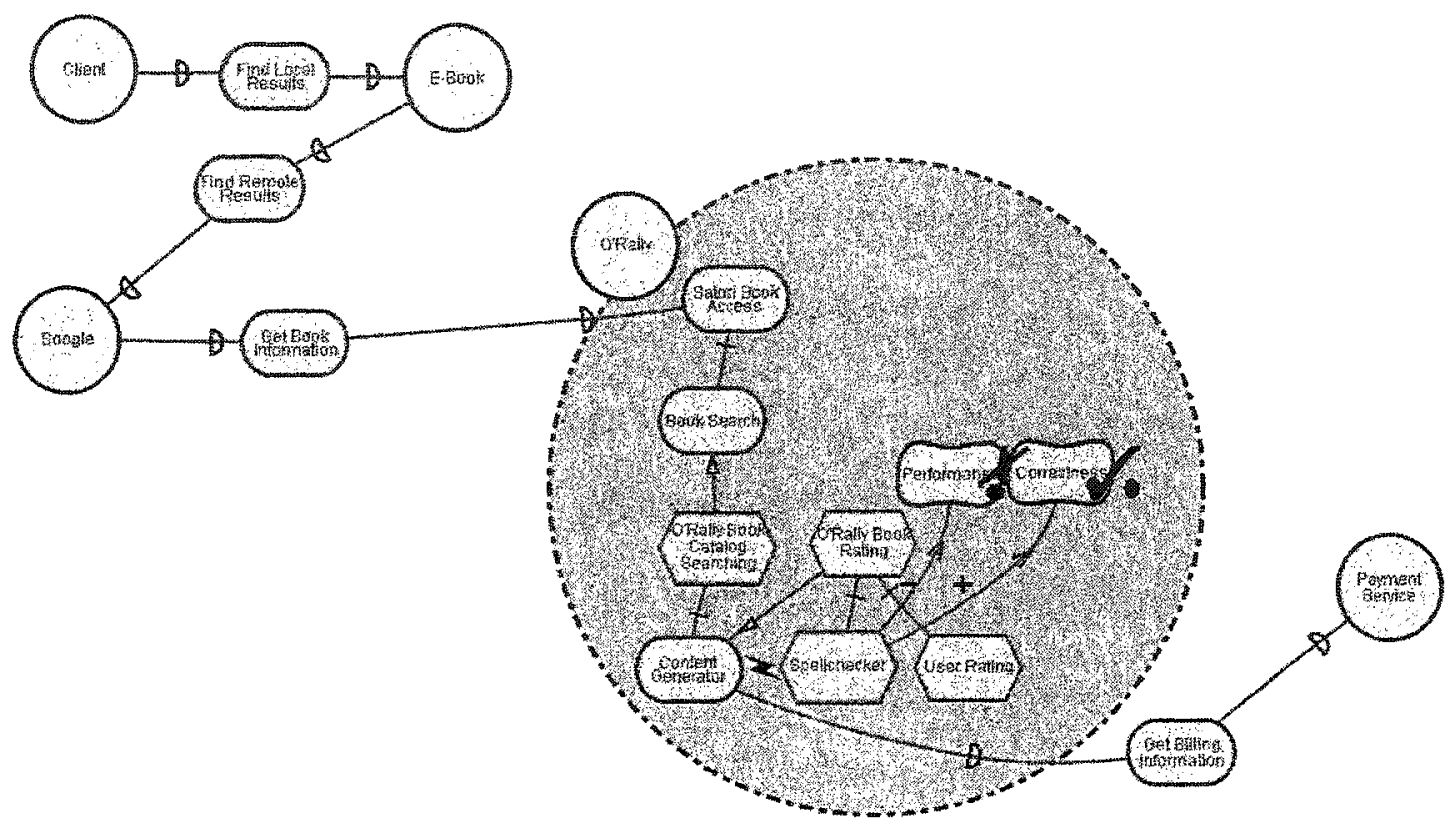

Figure 4.7: The GRL Model for an Interaction between ContentGenerator and

\section{Spellchecker}

As shown in Figure 4.7, in order to return the book rating and comments information to users, the Satori Book Service needs to get them from the O'Rally Book Rating Service. Before sending the comments to users, the spelling of the comments needs to be checked by a third party Spellchecker service. After that, the Satori Book Service will invoke the ContentGenerator feature of the O'Rally Book Catalog Service to generate book contents, rating information, and user comments for users. If the Spellchecker service is invoked before the invocation of the ContentGenerator feature, the contents of the books can be generated properly. However, if the ContentGenerator feature is invoked first, the contents may not be generated properly. 
Discussion: The feature interaction occurs between two functional requirements within a same actor: $O^{\prime}$ Rally. Therefore, it is a functional and internal interaction. This actor provides service to Boogle Search Service as a service provider. It also invokes Payment Service as a service consumer. Therefore, this actor acts in multiple roles: service provider and service consumer. Hence, it is a FIMR feature interaction. This feature interaction also impacts some non-functional concerns. The Spellchecker service improves the usability of the $O^{\prime}$ Rally Book Rating Service. However, it reduces the performance of this service.

When a service needs to invoke multiple services, the invocation order will be important to the service. Different invocation order may impact different non-functional concerns of the service or result in the incorrect result. In this example, as analysis above, If the ContentGenerator feature is invoked before the Spellchecking service, the contents may not be generated properly. Therefore, Different Invocation Order (see $\S 4.2 .3$ ) is the cause of this feature interaction.

\subsubsection{FESR (Functional-External-SingleRole Feature}

\section{Interaction)}

Definition 11 (FESR Feature Interaction). FESR feature interactions are feature interactions that occur among functional requirements within different actors. All actors act in a single role. They are different with FISR feature interactions. In this category, the feature interaction occurs in different scope. As defined previously, FISR feature interaction occurs in a single actor, therefore, the scope is limited in an actor, which can 
control all features involved in the feature interaction. On the contrast, FESR feature interaction occurs between two actors, and the scope is larger than FISR feature interaction. The actors are impossible to control all features involved in the feature interactions. Therefore, FESR feature interactions are more difficult to detect and resolve than FISR feature interactions. The Example 5.6 in Chapter 5 illustrates this category.

\subsubsection{FEMR (Functional-External-MultipleRoles Feature}

\section{Interaction)}

Definition 12 (FEMR Feature Interaction). FEMR feature interactions are feature interactions that occur among the functional requirements within different actors, and at least one of the actors acts in multiple roles. In comparison with FISR, FIMR, FESR feature interactions, this kind of feature interactions are the most complex ones because they have the natures of both larger scope and multiple roles. FEMR feature interactions are also hard to be detected by static detection methods because they are more possible occurring at the runtime. The solutions for them are limited because one actor is impossible to modify the features of the other actor. Especially, when the feature interactions occur in dynamic composition, these two actors involved in the feature interactions may not know each other. The following Example 4.4 illustrates this category. 
Example 4.4: Search Request Cache vs. Book Search

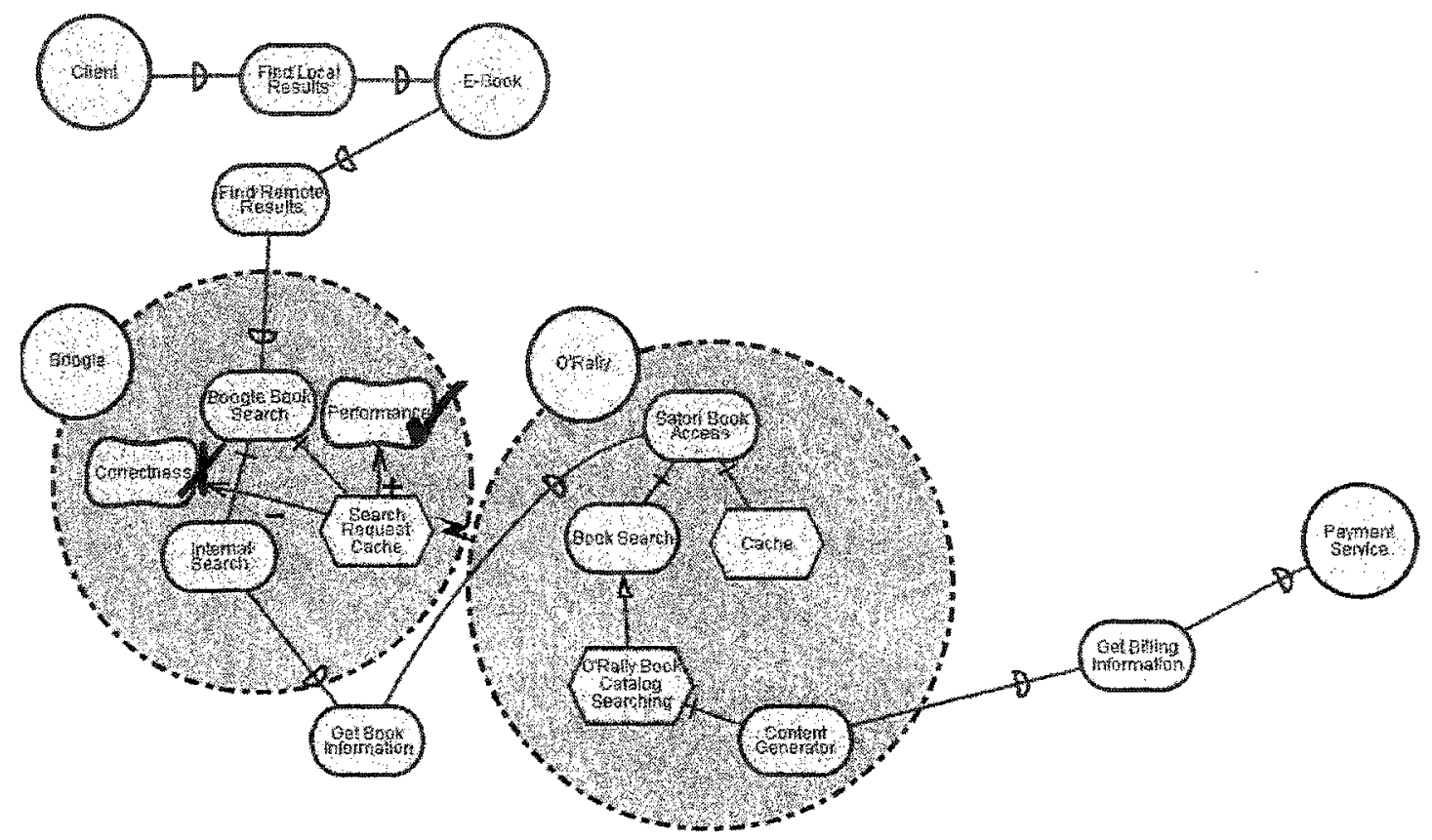

Figure 4.8: The GRL Model for an Interaction between the Features Search Request

Cache and Book Search

As shown in Figure 4.8, when the Local Book Search Service receives a client request, if it fails to find local results, it will redirect the request link to the Boogle Book Search Service. Then the Boogle Book Search Service will check its cache for this link. If this link does not exist in the cache, it will redirect this link to the Satori Book Service. Otherwise, it will not send the request to the Satori Book Service because it assumes a copy has existed in the cache on the client side. Therefore, for a new request link, the client can get the right results. If the client requests the same link again, it will fail to get results because the Boogle Book Search Service does not redirect this link to the Satori Book Service. The fact is the Satori Book Service will never store the contents of the 
books in the cache of the client. The contents of the book are always dynamically delivered via the Internet. That is a feature interaction between the Search Request Cache feature of the Boogle Book Search Service and the Book Search feature of the Satori Book Service.

Discussion: The feature interaction occurs between two functional requirements within two actors respectively. The Boogle Book Search Service acts as two roles: service provider and service consumer. Hence, this is a FEMR feature interaction. Furthermore, the Search Request Cache feature improves the performance of the Boogle Book Search Service by caching results. However, as presented in this example, it may result in an incomplete search result. Hence, it reduces the correctness of the Boogle Book Search Service.

The search request cache feature of the Boogle Book Search Service assumes the Satori Book Service will save a copy in the cache of the client, therefore, if the requested link has existed, it will not redirect the request again. That assumption causes this feature interaction. On the other hand, the Satori Book Service will not save a copy at the client side, and it always dynamically delivers the information to the client. This information is hidden from clients of this service. Therefore, we can infer two causes for this feature interaction: Violation of Assumptions about Service (see \$4.2.1) and Encapsulation and Information Hiding (see \$4.2.7). 


\subsubsection{NFISR (Non-Functional-Internal-SingleRole Feature}

\section{Interaction)}

Defimition 13 (NTISR Feature Interaction). NFISR feature interactions are interactions that occur among non-functional concerns within a single actor. Those interactions are side effects when functionalities have been implemented. The actor involved in the feature interactions acts in a single role. It is less studied and fewer supporting tools than FISR feature interactions. Therefore, it is more difficult to be detected and resolved than the FISR feature interactions. The Example 5.5 in Chapter 5 illustrates this category.

\subsubsection{NFIMR (Non-Functional-Internal-MultipleRoles}

\section{Feature Interaction)}

Definition 14 (NFIMR Feature Interaction). NFIMR feature interactions are interactions that occur among non-functional concerns within a single actor. The actor acts in multiple roles. Like the NFISR feature interactions, these feature interactions are also side effects produced by the implementation of functionalities. However, they are easier to occur and more complex than NFISR feature interactions. Unlike the solutions for FIMR feature interactions, the solutions for NFIMR feature interactions always impact other non-functional concerns. In fact, the solutions for those feature interactions will trade off all relative non-functional concerns. The following Example 4.5 illustrates this category. 
Example 4.5: Manageability vs. Trust

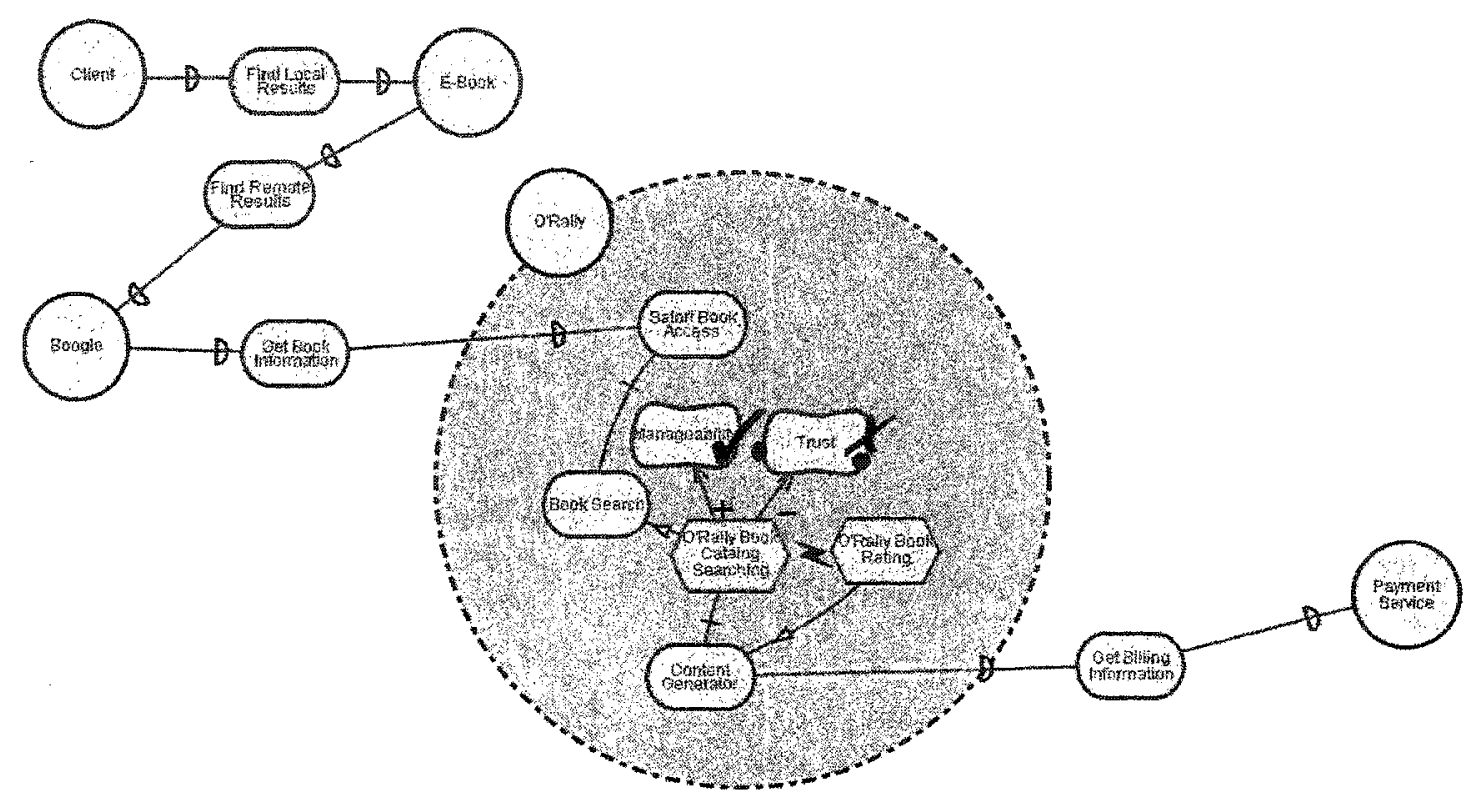

Figure 4.9: The GRL Model for an Interaction between Manageability and Trust

As shown in Figure 4.9, O'Rally Book Catalog Service and O'Rally Book Rating Service have a common owner. The O'Rally Book Catalog Service provides book information, which includes user comments and user rating. The $O^{\prime}$ Rally Book Rating Service can allow the user rate the books published by $O$ 'Rally. As the common owner of those two services, $O^{\prime}$ Rally can manage them easily. Therefore, this improves the manageability of O'Rally Book Catalog Service and O'Rally Book Rating Service. Meanwhile, O'Rally can very easily modify the book's rating in order to sell it. That defeats the trust of the O'Rally Book Rating Service.

Discussion: The interaction occurs between two non-functional concerns within a single actor: O'Rally. This actor provides the service to Boogle Search Service as a service 
provider. It also invokes Payment Service as a service consumer. Therefore, it acts in multiple roles. Therefore, it is a NFIMR feature interaction.

Same ownership improves the manageability of $O^{\prime}$ Rally Book Catalog Service and O'Rally Book Rating Service. However, the service owner is easy to modify the rating result. In this case, the trust of rating result will be defeated. $O^{\prime}$ Rally Book Catalog Service and $O^{\prime}$ Rally Book Rating Service conflict on interest. If they have the same owner, it will be a feature interaction. The cause for this conflict is Deployment and Ownership (see §4.2.6).

\subsubsection{NFESR (Non-Functional-External-SingleRole}

\section{Feature Interaction)}

Definition 15 (NFESR Feature Interaction). NFESR feature interactions are interactions that occur among non-functional concerns within different actors. All actors involved in feature interactions act in a single role. In comparison with NFISR feature interactions, NFESR feature interactions have a larger scope. This means that it is more difficult to trade off the non-functional concerns in NFESR feature interactions than in NFISR feature interactions. Although this kind of feature interactions has the same scope with FESR feature interactions, as interactions among non-functional concerns have not been as well studied as functional ones, NFESR feature interactions are more difficult to come up with than FESR feature interactions. The Example 5.7 in Chapter 5 illustrates this category. 


\subsubsection{NFEMR (Non-Functional-External-MultipleRoles}

\section{Feature Interaction)}

Definition 16 (NFEMR Feature Interaction). NFEMR feature interactions are interactions that occur among non-functional concems within different actors. At least there exists one of the actors acting in a multiple role. Feature interactions in this category are more difficult than other seven kinds of feature interactions for two reasons. The first reason is, as mentioned previously, feature interactions in non-functional concerns have been less studied than in functional requirements. Another reason is that NFEMR feature interactions are complex because of their larger scope and multiple roles. The Example 5.1 and Example 5.8 in Chapter 5 illustrate this category.

\subsubsection{Summary}

In this section, we use the following tables to summarize the relationship among examples, causes, and categories presented in the previous section.

\begin{tabular}{|c|c|c|c|c|c|c|c|c|c|c|c|c|c|c|c|}
\hline \multirow[b]{2}{*}{$\begin{array}{l}\text { Example } \\
\text { s }\end{array}$} & \multicolumn{8}{|c|}{ Nature of Feature Interactions } & \multicolumn{7}{|c|}{ Causes of Feature Interactions } \\
\hline & $\begin{array}{l}\text { FIS } \\
\mathbf{R}\end{array}$ & $\begin{array}{l}\mathrm{WI} \\
\mathrm{M} \\
\mathrm{R}\end{array}$ & $\begin{array}{l}\mathbf{F E} \\
\text { SR }\end{array}$ & $\begin{array}{l}\mathbf{F E} \\
\mathbf{M} \\
\mathbb{R}\end{array}$ & $\begin{array}{l}\text { NF } \\
\text { IS } \\
\text { R }\end{array}$ & $\begin{array}{l}\mathbf{N F} \\
\mathbf{I M} \\
\mathbf{R}\end{array}$ & $\begin{array}{l}\mathbf{N} \\
\mathbf{E S} \\
\mathbf{R}\end{array}$ & $\begin{array}{l}\text { NF } \\
\text { EM } \\
\text { R }\end{array}$ & $\begin{array}{l}\text { V } \\
\text { AS }\end{array}$ & $\begin{array}{l}\mathrm{DI} \\
0\end{array}$ & $\begin{array}{l}\mathbf{R} \\
\mathbf{C}\end{array}$ & $\begin{array}{l}G \\
C\end{array}$ & $\mathbf{P C}$ & $\begin{array}{l}D \\
0\end{array}$ & $\begin{array}{l}\mathbf{E} \\
\mathbf{H}\end{array}$ \\
\hline E4-1 & 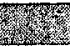 & & & & & & & & & & & & & & \\
\hline $\mathrm{E} 4-2$ & & W & & & & & & & & & & & & & \\
\hline E4-3 & & 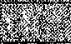 & & & & & & & & 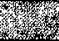 & & & & & \\
\hline $\mathrm{E} 4-4$ & & & & 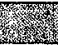 & & & & & 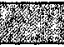 & & & & & & 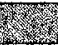 \\
\hline E4-5 & & & & & & Sivis & & & & & & & & 3re & \\
\hline $\mathrm{E} 5-1$ & & & & & & & & 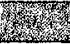 & & & & & & 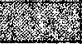 & \\
\hline $\mathrm{E} 5-2$ & & & & & & 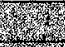 & & & 3is & & & & & & \\
\hline E5-3 & & & & & & 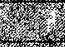 & & & & & & & & 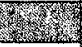 & \\
\hline E5-4 & & & & & & $x_{3}^{2}$ & & & & & & & & & \\
\hline E5-5 & & & & & 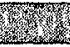 & & & & & & & 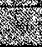 & 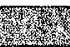 & & \\
\hline E5-6 & & & 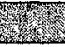 & & & & & & 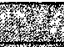 & & & & & & \\
\hline E5-7 & & & & & & & 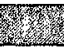 & & $\sqrt{2}$ & & & 4 & & & \\
\hline E5-8 & & & & & & & & & 2 & & & & & & \\
\hline
\end{tabular}

Table 4.2: The Relationship between Examples and Categories and Causes 
Notes for Table 4.2 and Table 4.3 :

1.

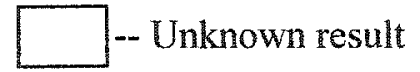

\section{Causes:}

VAS - Violation of Assumptions about Service; DIO- Different Invocation Order; RC-Resource Contention

GC-Goal Conflicts; PC - Policy Conflicts; DO - Deployment and Ownership; ElH - Encapsulation and Information Hiding

3.Categories:

FISR-Functional-Intemal-Single Role Feature Interactions

FIMR - Functional-Internal-MultipleRoles Feature Interactions

FESR-Functional-Extemal-SingleRole Feature Interactions

FEMR - Functional-External-MultipleRoles Feature Interactions

NFISR - Non-Functional-Internal-SingleRole Feature Interactions

NFIMR-Non-Functional-Internal-MultipleRoles Feature Interactions

NFESR-Non-Functional-External-SingleRole Feature Interactions

NFEMR-Non-Functional-External-MultipleRoles Feature Interactions

4.Examples:

E4-1-Example 4.1; E4-2-Example 4.2; E4-3-Example 4.3; E4-4-Example 4.4; E4-5-Example 4.5; E5-1-Example 5.1; E5-2-Example 5.2; E5-3-Example 5.3; E5-4-Example 5.4; E5-5-Example 5.5; E5-6-Example 5.6; E5-7-Example 5.7; E5-8-Example 5.8

Table 4.2 summarizes the relationship between examples and categories and causes.

In this table, each example only belongs to one classification. However, some examples

have more than one cause; that is why we can see more than one slot has been marked

with gray for those examples. However, the marked causes are not applied to all

examples in a specific classification because different examples in the same classification

maybe due to different causes. The collection of examples in this thesis just covers parts

of them. For example, E4-4 (Example 4.4) covers the category FEMR and two causes:

VAS and EIH. This example does not cover other categories and causes.

The following table summarizes the relationship between causes and categories:

\begin{tabular}{|c|c|c|c|c|c|c|c|c|}
\hline \multirow{2}{*}{$\begin{array}{c}\text { Causes of } \\
\text { Feature } \\
\text { Interactions }\end{array}$} & \multicolumn{8}{|c|}{ Nature of Feature Interactions } \\
\hline & FISR & FIMR & FESR & FEMR & NFISR & NETMR & NFESR & NELME \\
\hline VAS & 20 & (2) & 2 & 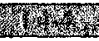 & & 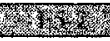 & X & 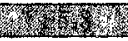 \\
\hline DIO & & (1) & & & & & & \\
\hline $\mathrm{RC}$ & & & & & & $4 \pi$ & & \\
\hline $\mathrm{PC}$ & & & & & & & & \\
\hline GC & & & & & & & & (x) \\
\hline $\mathrm{DO}$ & & & & & & 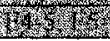 & & 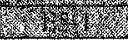 \\
\hline $\mathrm{EIH}$ & & & & 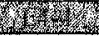 & & & & \\
\hline
\end{tabular}

Table 4.3: The Summary of the Relationship between Causes and Categories 
Table 4.3 summarizes the relationship between causes and categories. The slots, filled by examples and marked with gray, show the relationships between causes and classifications that have been covered in this thesis. The empty slots mean that the relationships between causes and categories have not been discussed. However, it is possible that feature interactions occur among them.

\subsubsection{Discussion}

Table 4.2 and table 4.3 summarize the relationships among examples, causes, and categories. Some examples may have multiple causes; therefore, they appear in more than one row. In the telecommunication domain, three different groups of approaches manage feature interactions [Cameron94]: infrastructure for deployment, design support, and run-time resolution. Each of these groups of approaches has to deal in some way with the avoidance, detection, and resolution of feature interactions. In the Web service domain, not much work has been done on detecting feature interactions.

In this thesis, based on the categorization of the previous section, a detection approach at the requirements stage will be given by analyzing dimensions and some of the causes of feature interactions presented in the previous section. We limit our approach to the requirements stage. If we can prevent feature interactions at an early stage, it will help prevent costly and time-consuming problem fixes during implementation.

The basic idea of the detection approach derives from the three dimensions and causes in the classification. For example: how to handle non-functional concerns? Which 
kinds of tools or framework can be used to analyze non-functional concerns? The GRL framework may be a good choice. We can use its soft-goals to present non-functional concerns while functional requirements can be presented by its goals. Although GRL goals can be used to describe functionality, it is too abstract to depict the further elaboration of design decisions. That means we need another tool or framework to capture the functional requirements. In the telecommunication domain, a number of tools for scenario modeling have been studied, such as MSC and UCM. In our approach, we choose UCM to perform scenario analysis because UCM is particularly suitable for analyzing deployment and ownership issues in feature interactions in the Web service domain. Therefore, those reasons lead a further step towards a detection approach presented in the next section.

\subsection{Towards a Detection Approach}

\subsubsection{Introduction}

As presented in Chapter 2 and Chapter 3, the GRL framework offers a goal-oriented analysis for a system. It allows us to answer questions about the design and resolve goal conflicts by using the goal graphs presented in Chapter 3 . Hence, we can address the feature interaction causes presented in the previous section by analyzing GRL goal graphs. If feature interactions occur between Web services in two actors, we will use dependencies to represent the relationship between them. If feature interactions occur between Web services in a same actor, we cannot use dependencies to represent the relationship between these Web services because an actor cannot support sub-actors in 
GRL model. Instead we use achieve links to model internal dependencies. It is different with UCM model. In UCM model, a component can include subcomponents.

UCM is also presented in Chapter 2 and Chapter 3. As stated in [L\&Y01], "Although goal-orientation is highly appropriate for requirements engineering, goals are sometimes too abstract to capture at once." Hence, a refinement of tasks in the GRL model will be handled by UCM. It will help GRL goal graphs to address causes. Another use of UCM is that it can be used to reason about the allocation of tasks/responsibilities to system components/actors. Subcomponents inside a UCM are used to represent multiple Web services within the same actor.

Hence, it is possible to detect and avoid feature interactions at the requirements stage of a system by combining GRL and UCM. The next section will present how to combine them together to detect feature interactions.

\subsubsection{Towards a Detection Approach}

Although Web services can be used in many fields, the most important field is the ebusiness application domain. This detection approach for feature interactions is a further development of an existing detection process presented in [W\&E04]. However, it is not a full detection approach. In Chapter 5 , an e-business system will be used as a case study to validate this approach. However, it does not mean that this detection approach can only work for the e-business application domain. In this thesis, we only concentrate on the ebusiness domain.

The detection approach comprises seven steps as shown in Figure 4.10. 


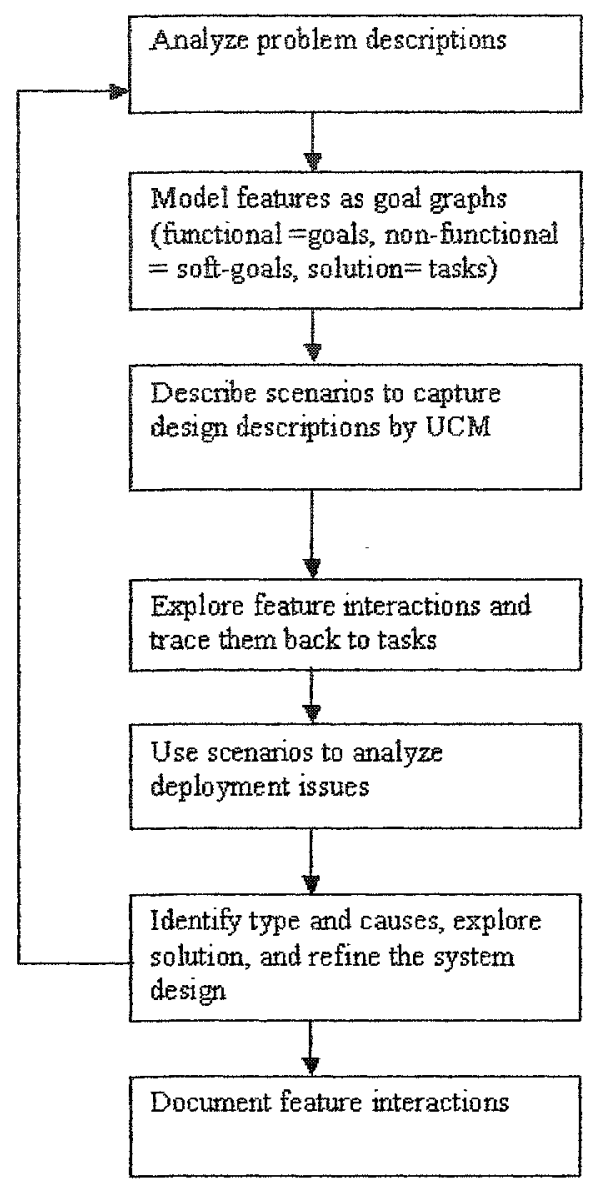

Figure 4.10: Steps of the Feature Interaction Detection Approach

\section{Analyze system scenarios}

This step will present the scenarios of the target application system. The scenarios include main functional requirements in the system, which are used to analyze feature interaction problem.

2. Model features as goal graphs (functional =goals, non-functional = soft-goals, solution=tasks)

In a real application system, non-functional requirements, such as performance, 
security, privacy, usability, are often crucial for the success of the system. In this step, we do not only describe functional requirements, but also non-functional concerns. Functional requirements will be presented as goals, while non-functional concerns will be presented as soft-goals. The tasks will be used to present the current solution.

\section{Describe scenarios to capture the design descriptions by Use Case Maps.} Use Case Maps provide a visual description of behavior in terms of scenarios (causal sequences of responsibilities along paths). It also provides a framework for making high-level architectural decisions. In this step, we will use UCM to model the system scenarios.

\section{Explore feature interaction and trace them back}

By analyzing the GRL model and the UCM model presented in step 2 and step 3 respectively, we can identify the various patterns to look for in the GRL/UCM models that could give rise to feature interactions. If the current solution causes a very negative influence for non-functional concerns or breaks the application system, we can say a non-functional interaction occurs with the current solution (tasks). Then we can track the scenario back to refine the solution.

\section{Use scenarios to analyze deployment issues}

This step is intended for reasoning about the allocation of tasks/responsibilities to system components/actors. 
6. Identify the types and causes of the feature interaction, and give the possible resolution for the feature interaction, and refine functional analysis.

In this step, first, we need to identify the types and causes of the feature interaction. Then we will give a possible resolution. After that, we need to modify our functional design and refine it. Each solution may introduce some new nonfunctional concerns conflicts when it resolves the old ones. Therefore, it is necessary to discuss the trade-offs with the solution in this step. We will repeat the above steps until we can get a satisfactory design system.

\section{Document the feature interaction.}

In this step, we will document the feature interaction as a reference for the future design with the following elements:

- Name-This item specifies the name of the identified feature interaction.

- Feature Interaction Category--This item categorizes the feature interaction into one of several possible categories specified in the previous section.

- Cause-This item presents causes of the feature interaction.

- Behavior-This item presents the function or service name in which feature interaction occurs.

- Web Service Stack Layer-We divide Web services stack [Sumra03] into three layers (Figure 4.11): Presentation Layer, Logic Layer, and Data Layer. We use this item to specify in which stack layer the feature interaction occurs. 


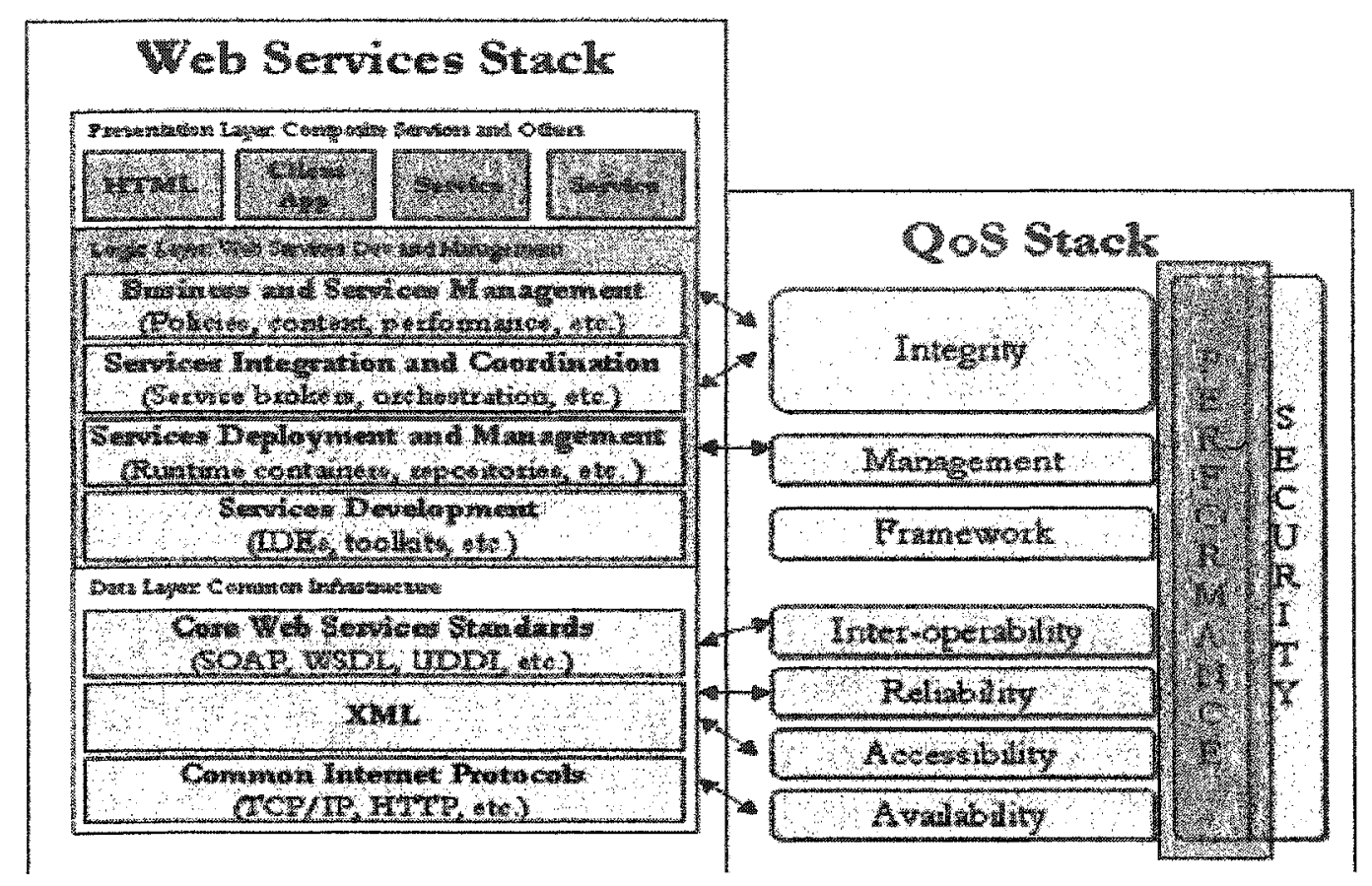

Figure 4.11: The Web services-QoS stack

\subsection{Chapter Summary}

In this chapter, we gave a classification of feature interaction in Web services based on their nature and causes. We identified three dimensions by their nature. We also got eight causes for feature interactions in Web services.

The second part of this chapter presented a further development of an approach for detecting feature interactions in Web services by combining the UCM and GRL notations. In this approach, first we will model features as goal graphs. Then, we will use UCM to analyze the scenarios of the system. Based on this analysis, we can infer possible functional or non-functional interactions. At the end of the approach, a solution for 
resolving the feature interaction and discussion of its trade-offs will be presented. One case study to verify this approach will be presented in the next chapter. This approach will be validated by using some common scenarios in an e-business system in Chapter 5 . Therefore, It also can be used to detect feature interactions in other e-business systems. 


\section{Chapter 5 Case Study: an E-Store}

\section{Example}

In order to verify the process and the classification presented in Chapter 4, a fictitious case study called the e-store system will be presented in this chapter. In this case study, login example is a real-world example while others are not real-world examples. However, it does not mean that they do not exist in the real Web service applications. Feature interactions in these examples can be easy to occur because of several common causes, such as infinitely loop. We use some fictitious examples to illustrate the existence of the type of feature interactions presented in Chapter 4 for two reasons. The first one is that the main purpose of this thesis is to classify feature interactions in Web services and present a detection approach instead of collecting feature interactions examples. The second reason is that real-world Web services are still very young. Therefore, it is difficult to explore the existing feature interactions in realworld Web services.

\subsection{The E-Store System Functionality}

The e-store system will be developed as an online e-business system consisting of book/music/video search, order, billing, and shipping functions. It performs those functions by interacting with other e-store systems or third parties service providers via Web services. Its main functional requirements include: 
- Login: provides functionality to let users login with an id and password. It mainly includes user authentication, authorization, and user profile management function.

- Search \& Display: offers functionality for valid users to search and display items online. The e-store will search the local catalog first. If no result can be found, the e-store system will search the result using Boogle Search Service. Then the other remote search provided by Remote Search Service will be invoked.

- Order Processing: allows valid users to place orders for the chosen items. This service places orders at a local service first. If local item quantities are less than the requested quantities, the service will search online and automatically place orders at remote services.

- Billing \& Shipping: provides functionality to calculate the cost for chosen items after customers have checked out. It will apply different discount policies for calculating the cost according to different customer membership types. It also calculates the tax for chosen items and shipping methods.

The following diagram depicts the functionality of the e-store system. The shaded ovals represent Web services, which will be used in this system. We will give more details about those Web services in subsequence sections. 


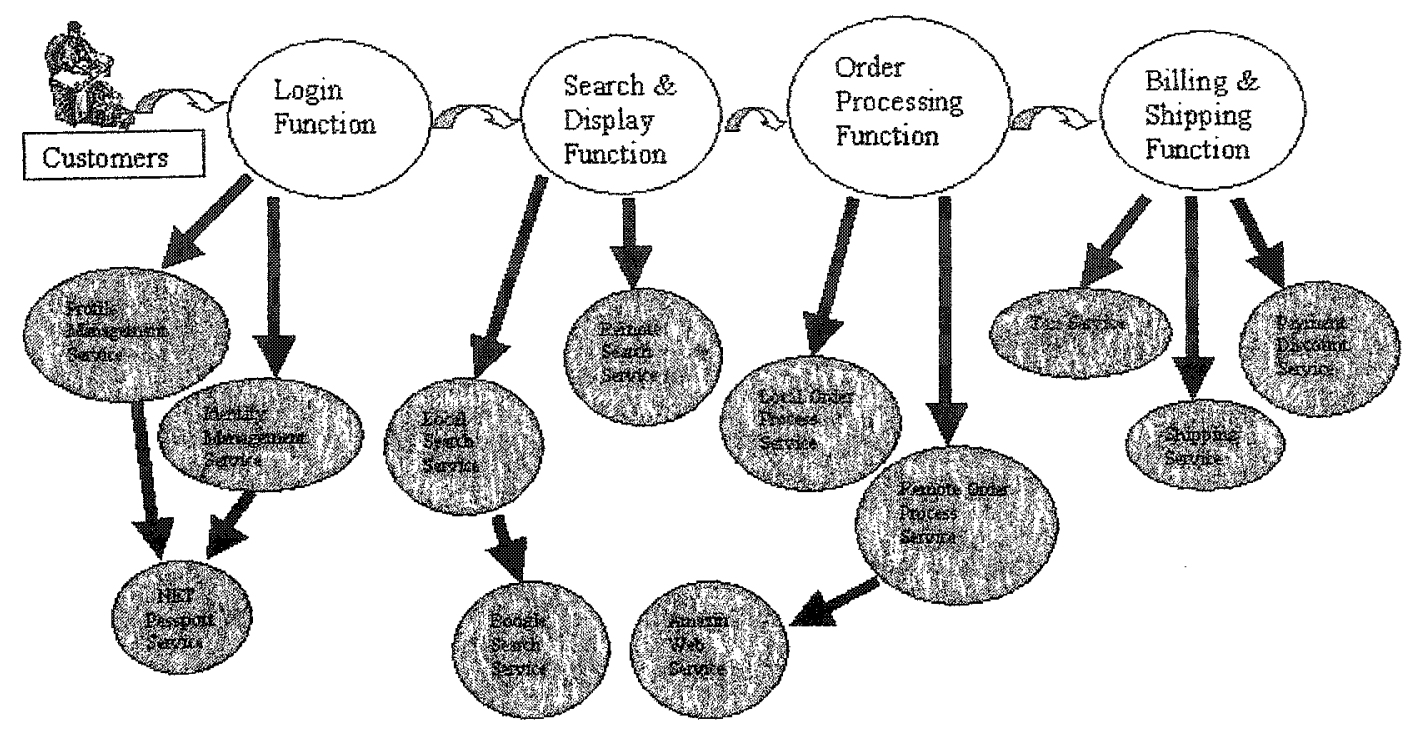

Figure 5.1: The Architecture of the E-Store System

\subsection{The E-Store System Analysis}

\subsubsection{A High-Level GRL Model of the E-Store System}

Consider a high-level GRL model (see Figure 5.2) of the e-store system: 


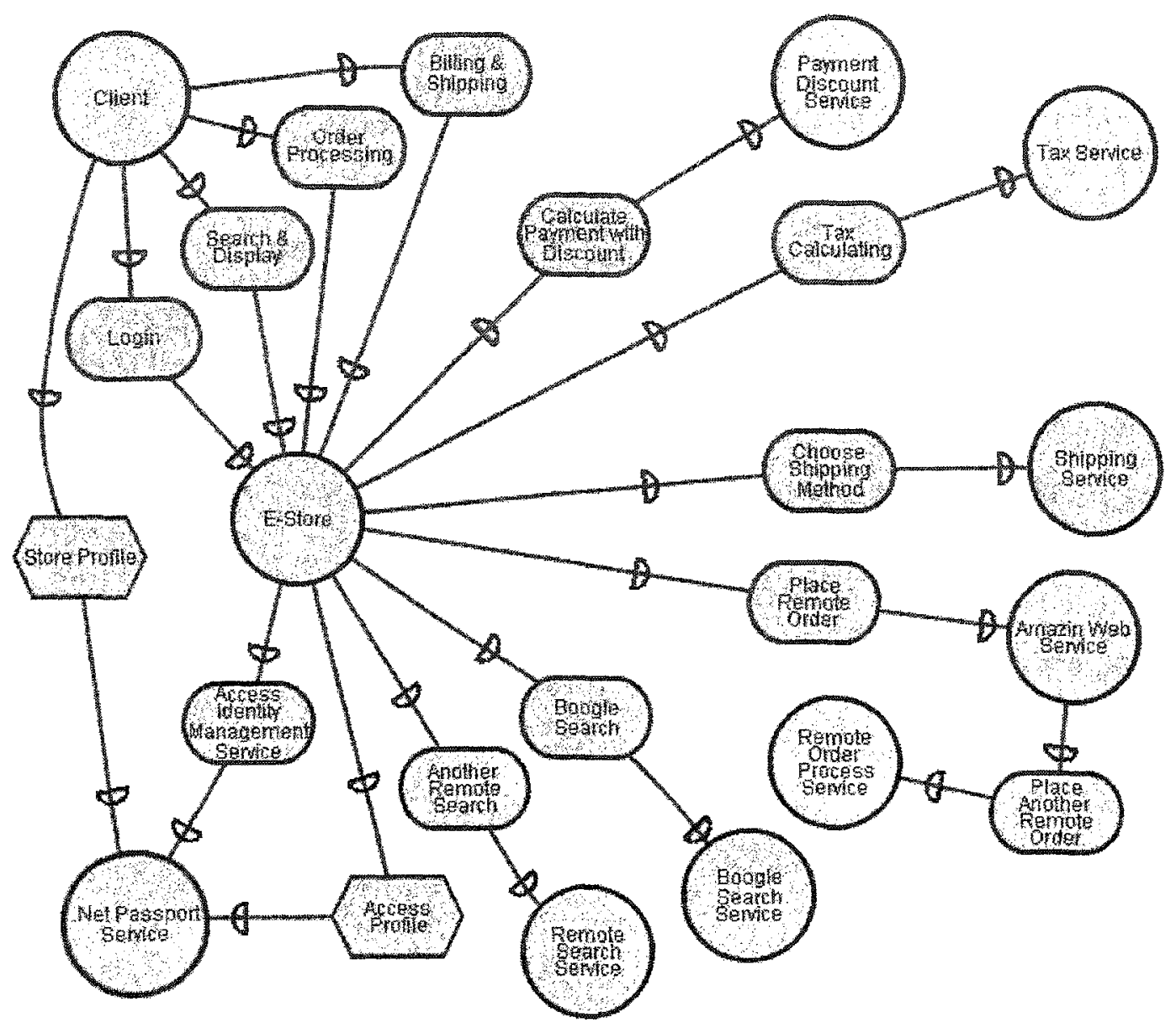

Figure 5.2: The High-Level GRL Model of E-Store System

As shown in Figure 5.2, there are ten actors: Client, E-Store, Net Passport Service, Boogle Search Service, Remote Search Service, Amazin Web Service, Remote Order Process Service, Payment Discount Service, Shipping Service, and Tax Service. The relationships among them are modeled as dependencies. For example, the Client depends on the E-Store for Login, Search\&Display, Order Processing, and Billing\& Shipping. 


\subsubsection{Root Use Case Map of the E-Store System}

Consider the highly abstract initial UCM of Figure 5.3.

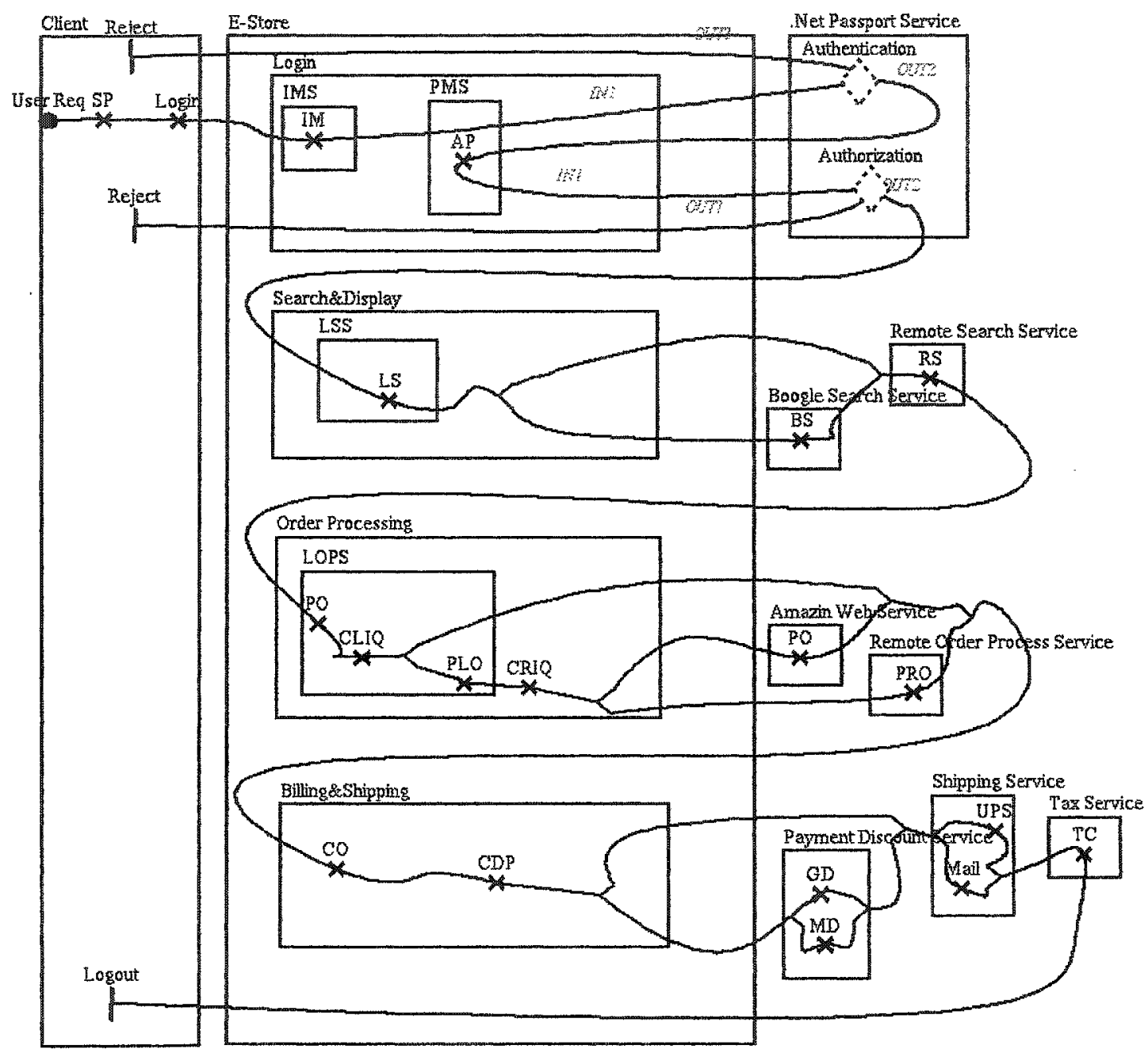

Figure 5.3: The Root Use Case Map of the E-Store System

\section{Notes for Figure 5.3:}

SP_-Store Profile; IMS-Identity Management Service; PMS - Profile Management Service; IM-Identity Management; AP--Access Profile; LSS - Local Search Service; LS-Local Search; BS-Boogle Search; RS - Remote Search; LOPS-Local Order Process Service; PO-Process Order; CLIQ-Check Local Item Quantities; PLO-Place Local Order; CRIQ - Check Remote Item Quantities; PRO-Place Remote Order; CO-Check Out; GD-Group Discount; MD - Member Discount; TC-Tax Calculation; 
As shown in Figure 5.3, there are two dynamic stubs, which are Authentication and Authorization. Each stub as a place holding for multiple plug-ins represents Login functionality. We implement the Login function by combining the Identity Management Service (IMS) and the Profile Management Service (PMS). We use the Microsof .NET Passport Service (NPS) to implement the identity management service. The e-store Search \& Display function invokes the Local Search Service (LSS), Boogle Search Service (BSS), and Remote Search Service (RSS). The e-store Order Processing function includes the Local Order Process Service (LOPS). It may invoke the Amazin Web service (AWS) to place a remote order if local quantities are not enough. The AWS may invoke other Remote Order Process Service (ROPS) to place the order if the quantities are not enough. The e-store Billing \& Shipping function will invoke the Payment Discount Service (PDS) with different discount policies, Tax Service (TS), and Shipping Service (SS) with different shipping methods.

The scenario is that the user requests to be authorized by the NPS first. If it is successful, he/she searches the items with the Search \& Display function. If the user finds the record at local, the system will display it directly. Otherwise, the system will automatically search the items by the BSS. Then it will invoke other RSS to search the items at remote catalog. After valid items are found, the user needs to enter the quantities of items. If local item quantities are more than requested items, the LOPS will place a local order for the user. Otherwise, the service will place a local order for the user first, and then it will calculate the difference of the quantities between the user entered and the locally available. After that, it will invoke the $A W S$ to place a remote order for the extra quantities. If the quantities are still not met, the e-store will invoke other ROPS to place 
an order until the quantities are met. When the user checks out, the PDS will be invoked first to calculate the cost for the user by applying different discount policies. The user can choose the different shipping methods by invoking the $S S$, and finally the tax will be calculated by the TS.

\subsubsection{A Refined GRL Model of the E-Store System}

The Figure 5.4 is a refinement of Figure 5.2. As shown in Figure 5.4, beside these functional requirements presented above, some non-functional concerns are also presented and explained in the next section. We choose these non-functional concerns depending on whether they are concerned by the system or users. For example, users consider whether it is safe to provide their personal information to the e-store system. Therefore, privacy will be a concern of the users. The e-store system considers whether its services are easy to be used. Whether can its services cooperate with other third party services? How about is the whole system performance? Whether is the whole system easy to be maintained? The answer of those questions decides which kinds of nonfunctional concerns need to be chosen. These non-functional concerns will be affected by the implementations of the functional requirements. In this thesis, "6" denotes that the implementation helps the non-functional concern, and "s"s denotes that the implementation hurts the non-functional concern. These functional requirements and nonfunctional concerns will be used by the detection approach presented in Chapter 4 to detect feature interactions in the Web service domain. Meanwhile, some of the categories and causes presented in Chapter 4 will also be verified by the feature interactions occurred in those features in the next section. 


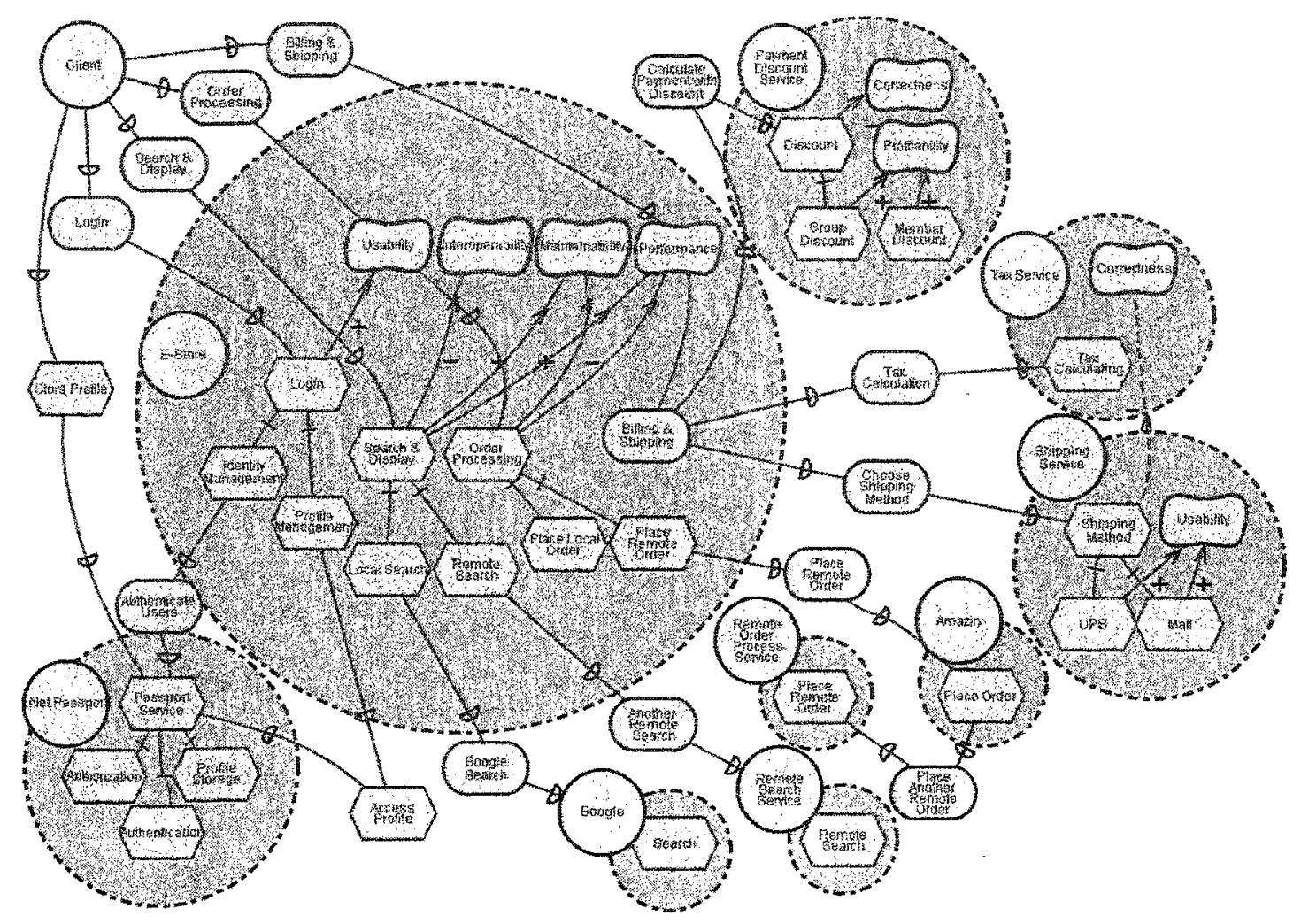

Figure 5.4: A Refined GRL Model of the E-Store System

\subsubsection{A Refined Use Case Map model for the E-Store}

\section{System}

A detailed UCM refines the root map of this system (see Figure 5.5). This refined UCM model just gives the difference with the Figure 5.3. It gives a detailed map for two dynamic stubs in Figure 5.3. In this map, when the system receives a request from a user, it will redirect the request to the .Net Passport Service (NPS). If the user is a valid user, he/she can be authorized to do the other operations in the e-store system. 


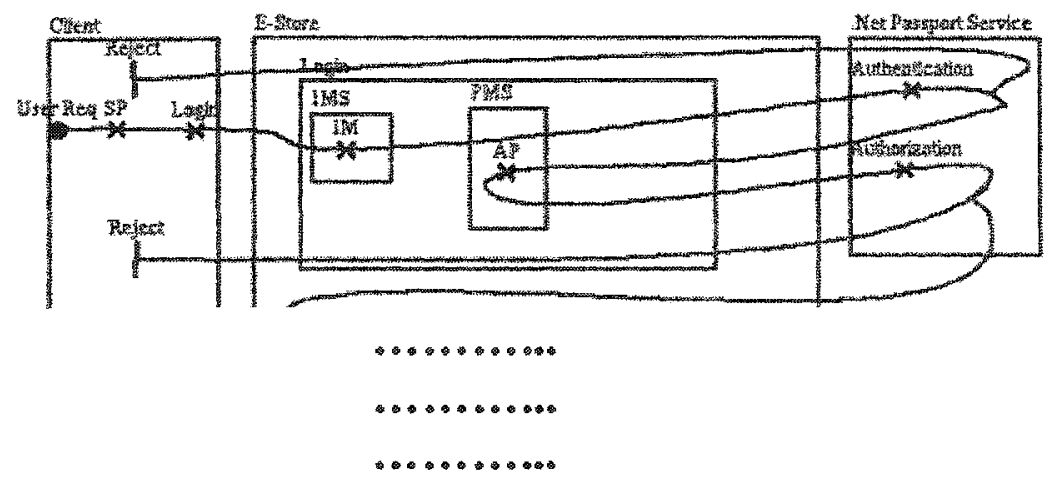

Figure 5.5: A Refined Use Case Map of the E-Store System

Notes for Figure 5.5:

IMS-Identity Management Service; PMS - Profile Management Service;

IM-Identity Management; AP- Access Profile;

\subsubsection{Web services in the E-Store System}

This section will give descriptions of each Web service presented in this chapter.

Some features of these Web services used in the e-store system will also be presented.

1) Profile Management Service

This service is used to access the user profile. One feature will be presented in the e-store system.

- Access Profile Feature

This feature is used to access user profiles.

2) Identify Management Service

This service provides user identify management function. It can be implemented by other Web services. In this example, it provides a feature to invoke other identify management services.

- Identity Management Feature

The e-store system uses this feature to authenticate users. 
3) .Net Passport Service

This service can be used to implement the identify management function of the estore system. There are two features in it:

- Authorization Feature

This feature authorizes users to access the e-store system.

- Authentication Feature

This feature verifies whether the user has been authorized.

4) Local Search Service

This service provides feature to search items in the e-store local catalog. There is one feature in it:

\section{- Local Search Feature}

This feature is used to search items in the local catalog.

5) Boogle Search Service

If local search failed to find the required items, the Boogle Search Service will be invoked automatically to perform the Boogle search. It includes one feature:

- Boogle Search Feature

This feature is used to search required items by Boogle. Once it receives the user's request, it will pass the query request to the marketplace, which will redirect the request to the supplier using a new SOAP service request. However, the supplier is only able to respond to catalog queries using another vocabulary set, such as the RosettaNet [RosettaNet03] vocabulary, which is different from the vocabulary set of the Local Search.

6) Remote Search Service 
Regardless of the search results of the Local Search and the Boogle Search, the estore system will invoke the Remote Search Service to search items in the other remote catalogs. This service includes one feature.

- Remote Search Feature

This feature is used to perform remote search.

7) Local Order Process Service

If the e-store system can find the required items at the local catalog, it will use this service to place a local order. One feature will be used by the e-store system in this service:

- Local Order Process Feature

It is used to place a local order.

8) Remote Order Process Service

If the local quantities of the items are less than those required by the user, the estore system will redirect the request to Amazin Web Service to place a remote order.

\section{- Place a Remote Order Feature}

This feature is used to redirect the request to the Amazin Web Service to place a remote order.

9) Amazin Web Service

It is used to place a remote order. If the quantities of the items are less than those required by the user, it will call other Web services to place another order.

- Place Order Feature

This feature is used to place an order to Amazin. 
10) Tax Service

This service is used to calculate the tax according to a single standard when the user checks out. The amount depends on shipping method and items ordered.

- Tax Calculating Feature

This feature is used to calculate the tax for the chosen items and shipping method.

11) Shipping Service

The e-store system provides two choices for the shipping.

- UPS Feature

This feature means the shipping will be done by UPS. If the user chooses this shipping method, the e-store will not calculate the chosen items' tax.

- Mail Feature

This feature means the shipping will be done by Mail. If the user chooses this shipping method, the e-store will calculate the chosen items' GST and PST.

12) Payment Discount Service

This service is used to calculate the payment discount. It includes:

- Group Discount Feature

It calculates the payment discount for the group members. It gives $15 \%$ discount for the group members.

- Member Discount Feature

It calculates the payment discount for the VIP member. It gives $30 \%$ discount for the VIP members.

Some other Web services used in the solutions will not be presented in this section. 


\subsection{Feature Interactions in the E-Store System}

\subsubsection{E-Store Login Function}

We will refer to those seven steps defined in Chapter 4 to analyze the feature interactions. In those seven steps, first, we need to analyze the scenarios. Second, we need to model features as goal graphs and need to describe the scenarios using the UCM. After this, we can explore feature interactions and deployment issues by analyzing GRL and UCM models. At the end of the process, the feature interactions will be documented.

\section{Step 1: Analyze Problem Descriptions}

The e-store system is a participating . NET Passport site. It will authenticate the users with a common Internet authentication mechanism. The Login function scenario is the following:

- A user inputs username and password on the e-store login screen and requests authentication.

- The Login function receives the user's input, and redirects the request to the NPS.

- NPS presents the user with a login page over a SSL connection.

- The user logs into the NPS and the NPS redirects the user back to the Login function. Authentication information is included in the redirected response inside the query string.

- The Login function sets an encrypted cookie in the client's browser.

\section{Step 2: Model Features as Goal Graphs}




\section{Features}

- Authentication

- Authorization

- Authenticate Users

- Access Profile

\section{Non-Functional Concerns}

- Usability

Usability is the measure of a Web service's potential to accomplish the goals of the user. Some factors used in determining Web service usability are:

- Ease-of-use. That allows customers to quickly and simply accomplish what they want to do.

- Visual consistency. Pages appear as parts of the same whole.

- Flexibility. A Web service can enhance its flexibilities by providing multiple choices for the user. This can also attract the user to be back to use the service again. This improves the service's usability.

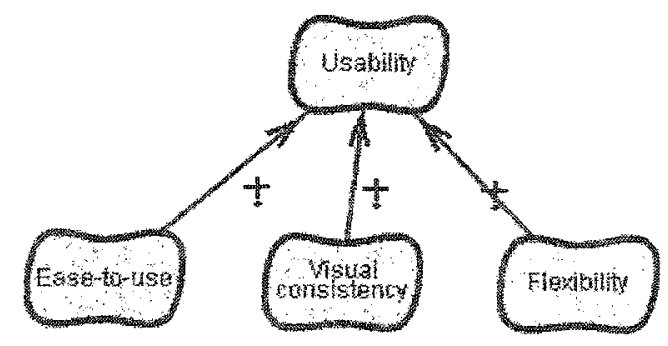

Figure 5.6: Force Hierarchy for Web Services Usability

- Privacy 
"Privacy refers to aspects of individuals or entities that the owner wants to remain confidential from third-party interests "[Ghosh01]. It includes three aspects:

- Data. All documents the individual wishes to keep confidential.

- Properties. They include those defining aspects of the individual or entity, such as physical attributes and preferences, such as religious faith.

- Behavioral characteristics, such as the schedule we keep and our shopping habits.

From a customer's perspective, there are at least three main privacy concerns:

- What personal information can be shared;

- Whether messages can be exchanged without anyone else seeing them;

- Whether and how one can send messages anonymously;

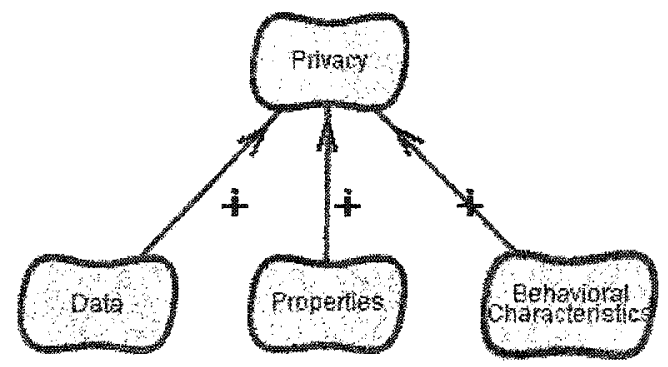

Figure 5.7: Force Hierarchy for Web Services Privacy

We can make the Login function easy to use by using the PMS and IMS [Weiss03]. Hence, we can say these two services enhance the usability of the e-store system. 
In the e-store system, Some existing Web services will be used to implement identity management, such as Microsoft .NET Passport [Passport04] [Kormann00] [Slemko01].

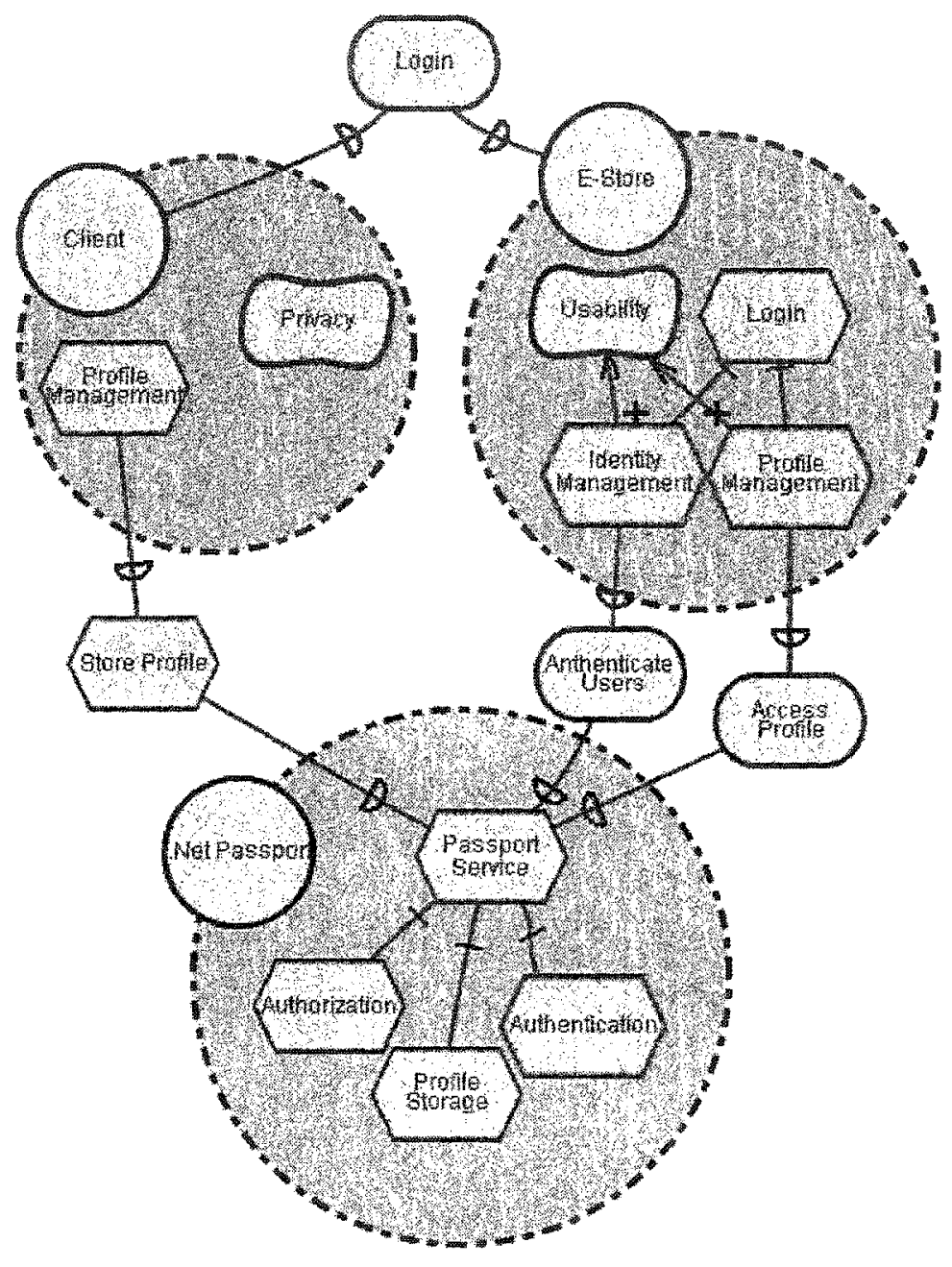

Figure 5.8: Using Two Services to Improve the Usability of the System

\section{Step 3: Describe Scenario by UCM}

The following UCM model gives a detailed description of the login scenario. In this scenario, there are two components into the e-store login: the $P M S$ and the $I M S$. When the e-store system receives the user request, if will pass this request to the NPS by 
feature IM deployed in the IMS component. The e-store system then checks the user's username and password by the NPS. If the e-store verifies the user, it can access the user profile by feature Access Profile (AP) deployed in the PMS component. The NPS will authorize the e-store to access the user profile. In the following figure, the features Authorization and Authentication are deployed in the NPS component.

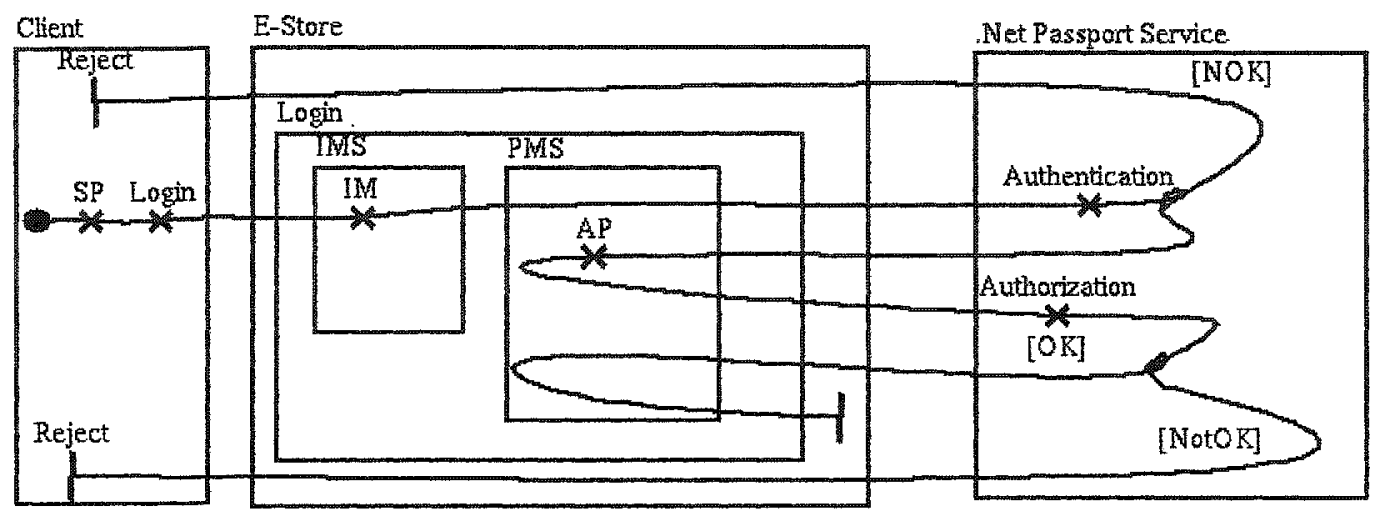

Figure 5.9: Detailed Maps for Login

Notes for Figure 5.9:

SP - Store Profile; IM - Identity Management; IMS - Identity Management Service; PMS - Profile Management Service; AP_- Access Profile

\section{Step 4: Explore Feature Interactions}

\section{Example 5.1: Usability vs. Privacy}

In this scenario, NPS offers authentication and authorization features. The authentication identifies the user to the service provider. However, the authorization gives the service provider access to the user's profile without the user's permission. If privacy matters to the user, the user's context must always be considered. If using the NPS as identity management, the user's context will not be considered. Hence, we can say the NPS violates user privacy if the user considers that the privacy is important. 


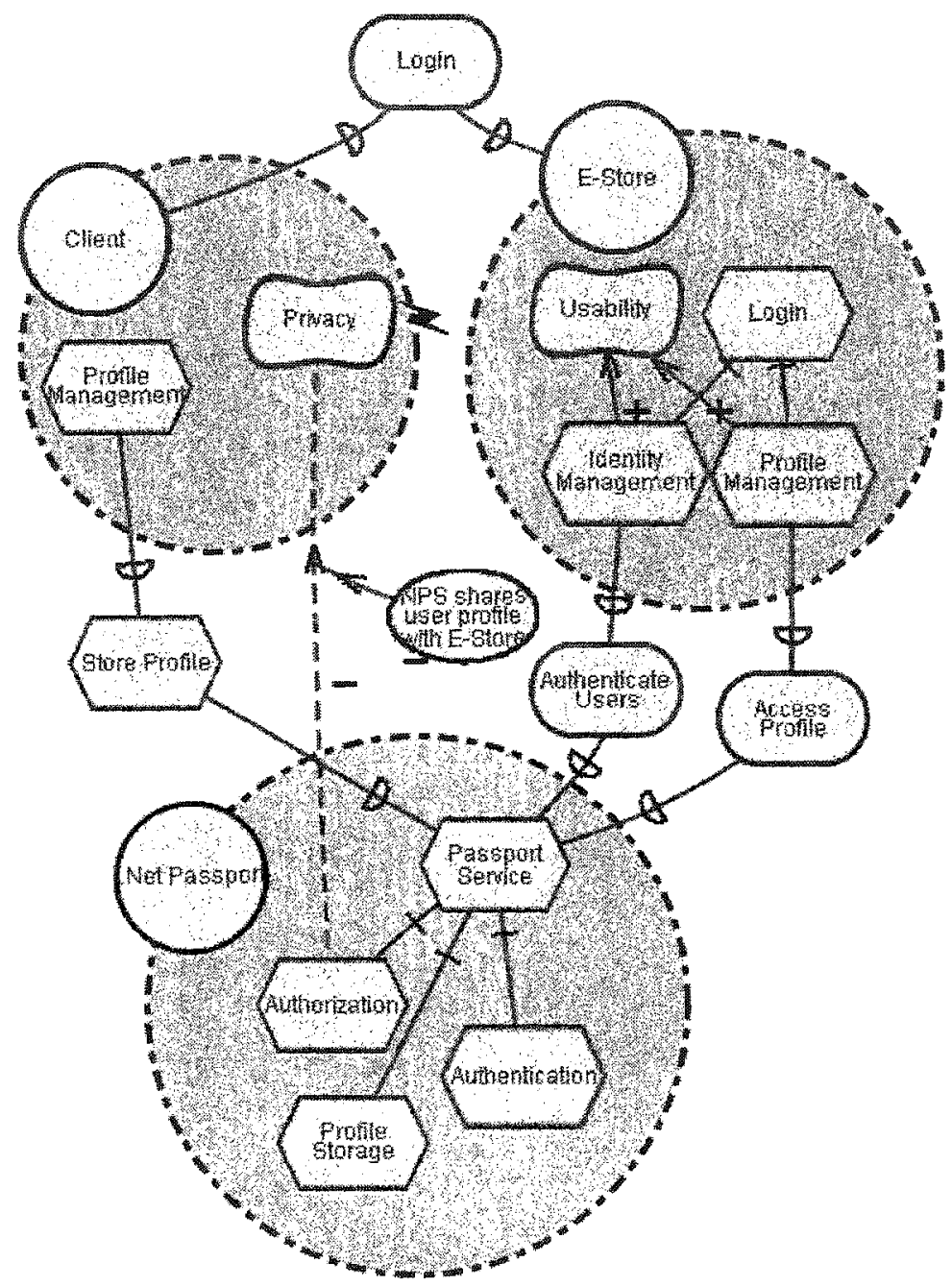

Figure 5.10: Non-Functional Interaction between Privacy and Usability

We can verify this feature interaction at NET Passport test mode. From the following figures, we infer that every participating .NET Passport site can read the user's profile. 


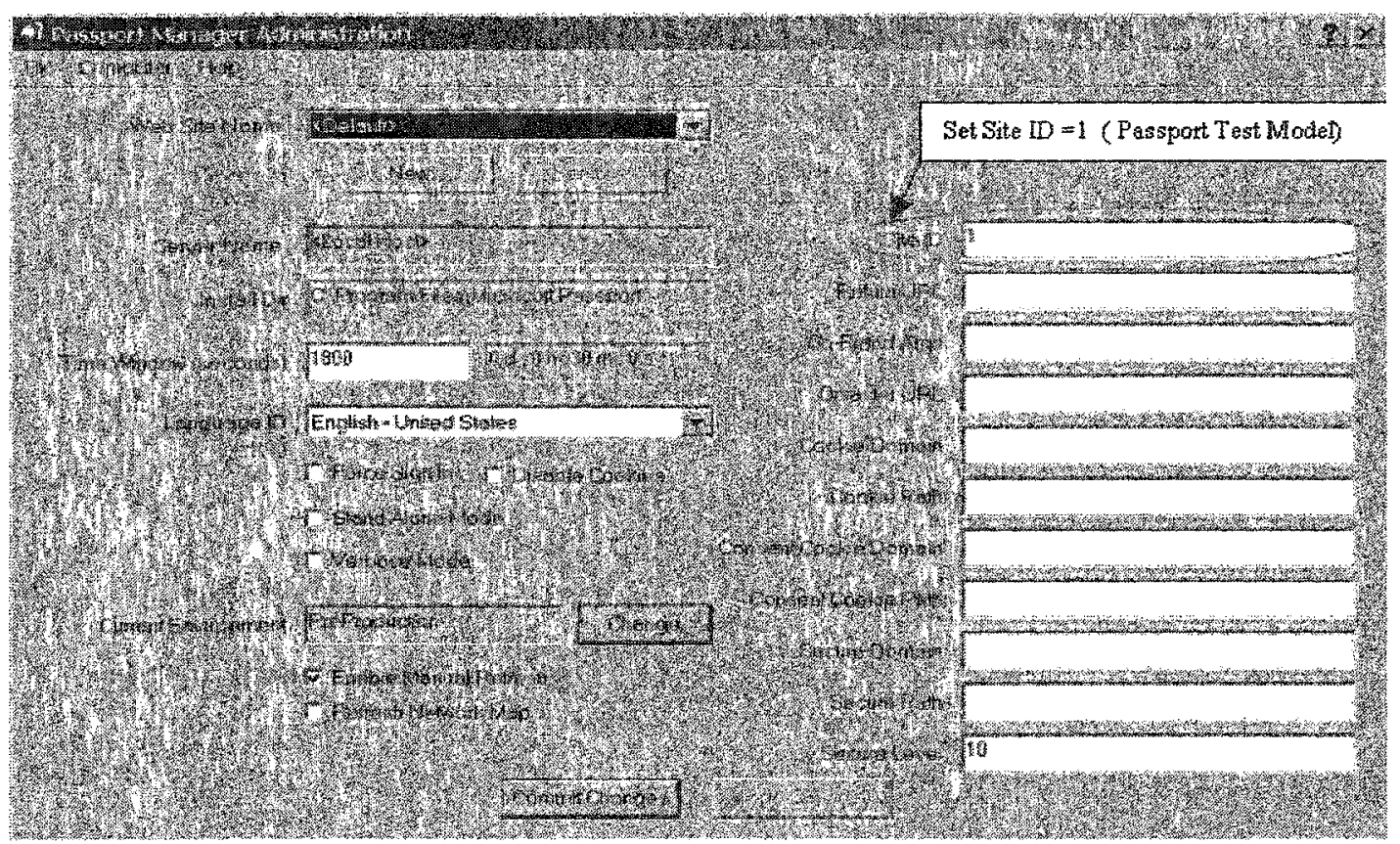

Figure 5.11: Set the .NET Passport Work Mode to Test Mode (Site ID =1)

In this example, the test site is a service provider. It is a .NET Passport service participating Web site. In other words, it uses the .NET Passport service to authenticate the potential service consumer. Before we run it, we need to set the .NET Passport service work on the test mode by specifying the siteID $=1$ in .NET Passport manager administration (see Figure 5.11). The .NET Passport manager administration is an administration tool provided by Microsoft.

After we set.NET Passport work on the test mode, we then run the test site at the browser by entering "http://your websever IP Address/PassportTest/" at the address bar of Internet Explorer. Then the test site displays the first page (see Figure 5.12). In this page, the test site displays that the user is not authenticated. The user is required to login by clicking the "Sign in" button. This button just redirects the request to the .NET Passport sign-in screen. 


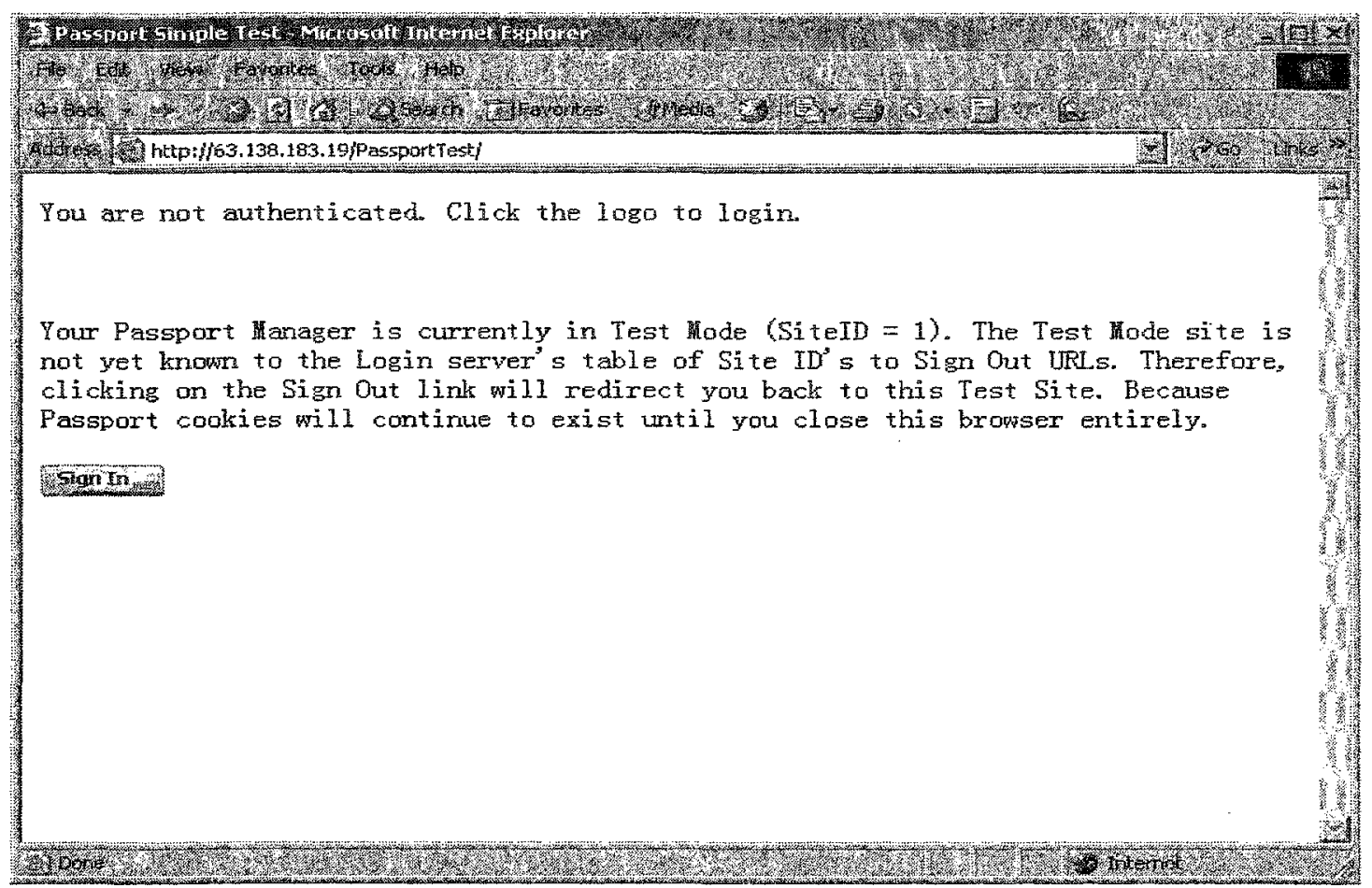

Figure 5.12: Runs the Test Site

When the user clicks the "Sign in" button, the test site will display the .NET

Passport sign-in screen. The user is required to enter the e-mail address and password in this screen (see Figure 5.13). 


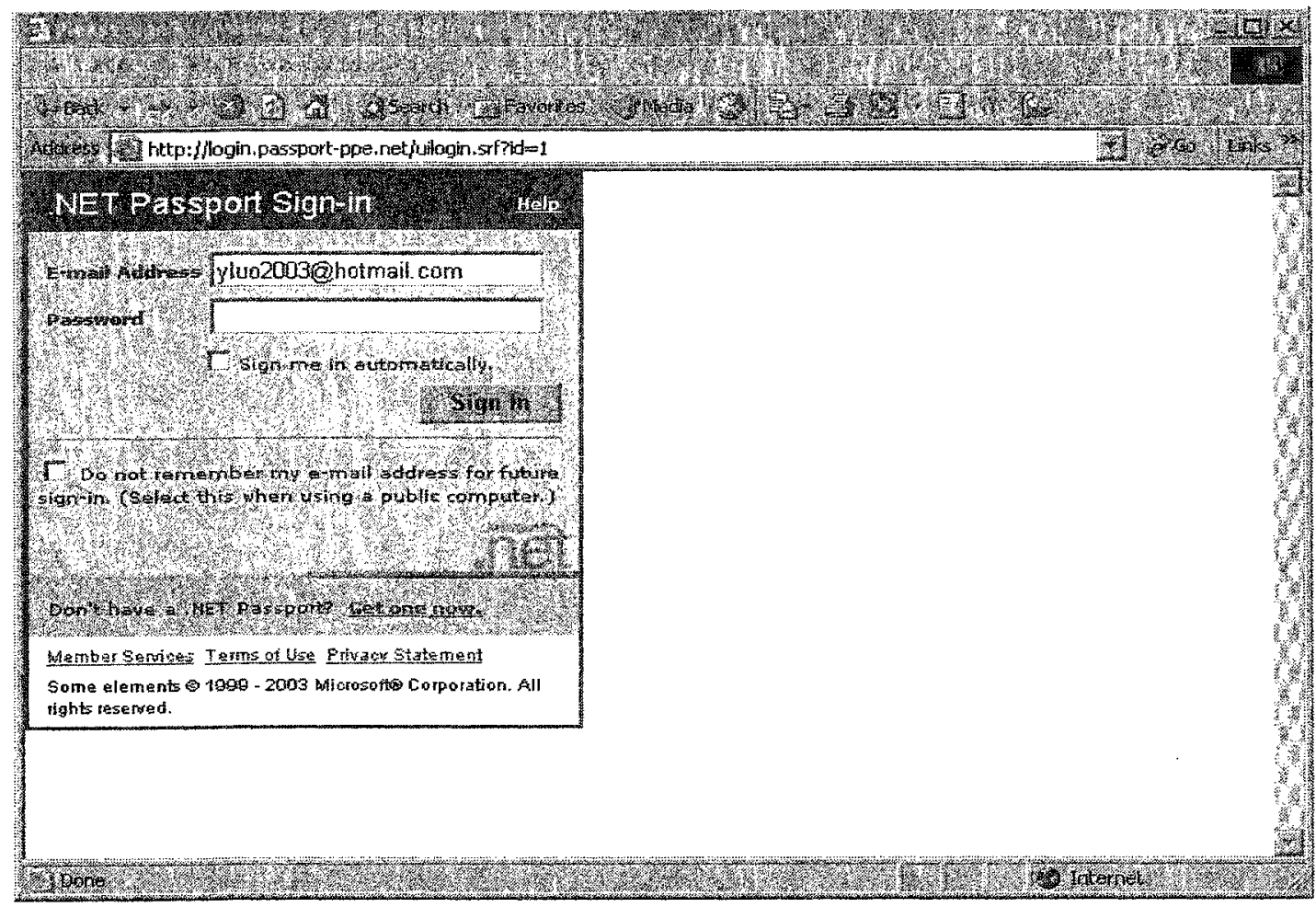

Figure 5.13: Enters the Email Address and Password, and Clicks "Sign in" Button

After the user clicks "Sign in" button, the user's profile is shown at the test site (see Figure 5.14). This is not right. The user is only supposed to login the test site by the NPS and access the contents of test site instead of his/her profile. The user just can read and maintain his/her profile in Microsoft Web site instead of all participating Web sites of the NPS. This proves that the owner of the NPS shares the user's private information with the test site. In other words, the Microsoft NET Passport Service shares the user profile with its participating Web sites without the user permission. This violates the user privacy. 


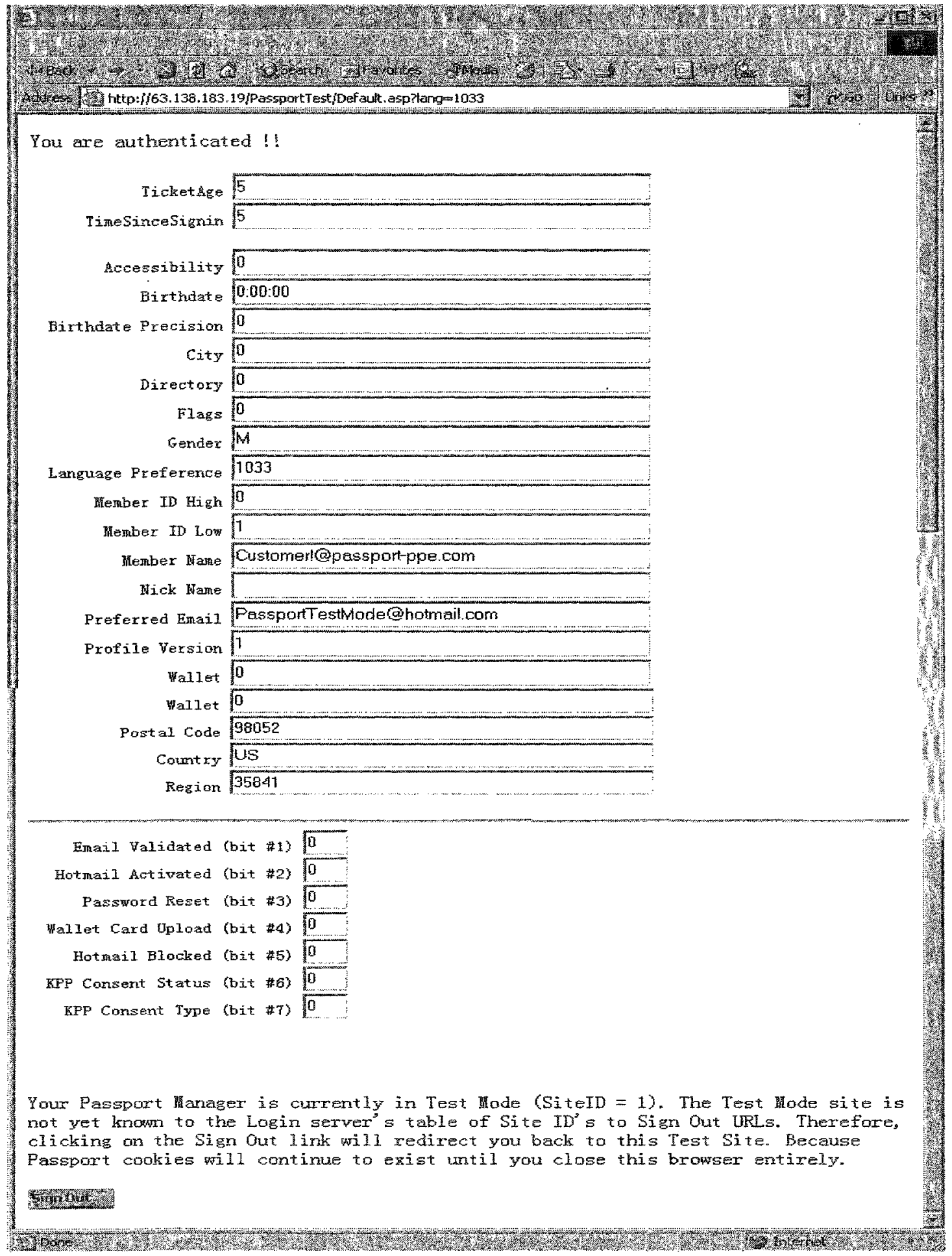

Figure 5.14: The User (service consumer) should not have Gotten his/her Profile from the Test Site (service provider) 


\section{Step 5: Using UCM to Analyze Deployment Issues}

As the above analysis shows, this design could not satisfy the non-functional requirements because the ownership of authorization and authentication cannot be the same. The following UCM model shows this problem.

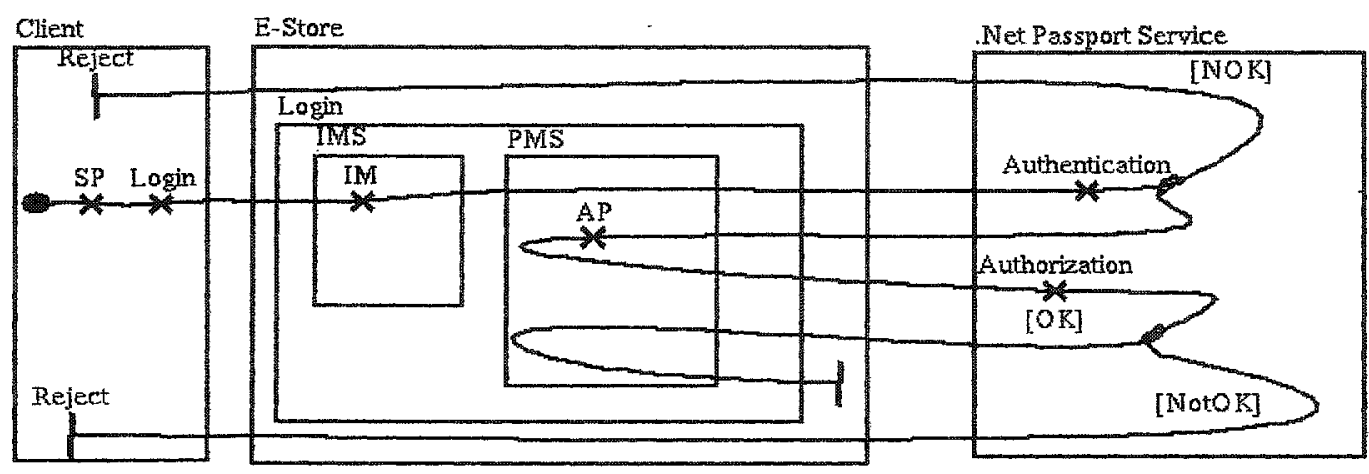

Figure 5.15: Using Use Case Map to Analyze the Deployment Issue

Notes for Figure 5.15 and Figure 5.16:

SP-Store Profile; IM- Identity Management; IMS - Identity Management Service;

PMS - Profile Management Service; AP - Access Profile

\section{Step 6: Solution and Discussion}

One solution is to separate the authorization and authentication features. As shown in Figure 5.16, the authorization has been moved to the component Client. We will use another Identify Management Service to replace the NPS to perform the authentication.

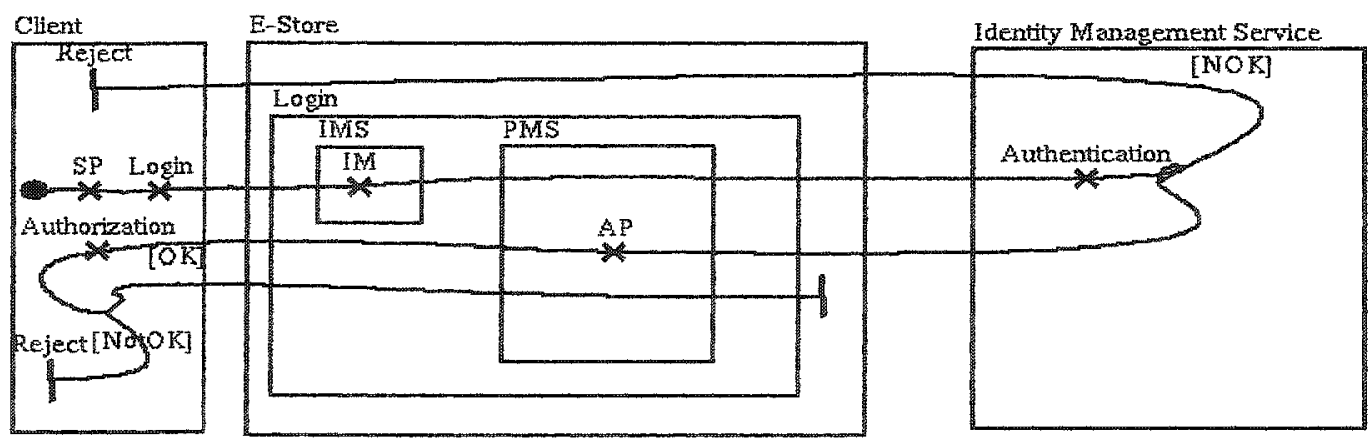

Figure 5.16: Refined Use Case Map to resolve the Deployment Issue 
As the above analysis, interaction occurs between non-functional concerns: Privacy and Usability. It occurs in two actors: Client and E-Store. The actor E-Store acts as two roles: service consume and service provider. Therefore, the feature interaction is a NFEMR feature interaction. As analysis above, authorization and authentication cannot be deployed in one service. Therefore, the Deployment and Ownership (see $\S 4.2 .6$ ) is one of the causes of this feature interaction. Goal Conflicts (see $\$ 4.2 .5$ ) is another cause of this feature interaction.

With the change of UCM model, the GRL model also needs to be changed. The following GRL model describes a solution. In this solution, we decouple the authentication and authorization features. Furthermore, the authorization feature should be implemented in accordance with the P3P protocol [P3P98]. This can let the user control his information.

This solution protects the user's privacy. However, it may reduce the system's efficiency because the user's profile is not stored by the NPS. Instead, the user's profile is stored and managed by the client. This means the user may have to register and provide his/her personal information to every website he/she wants to access, unless the client program provides helps with profile management. 


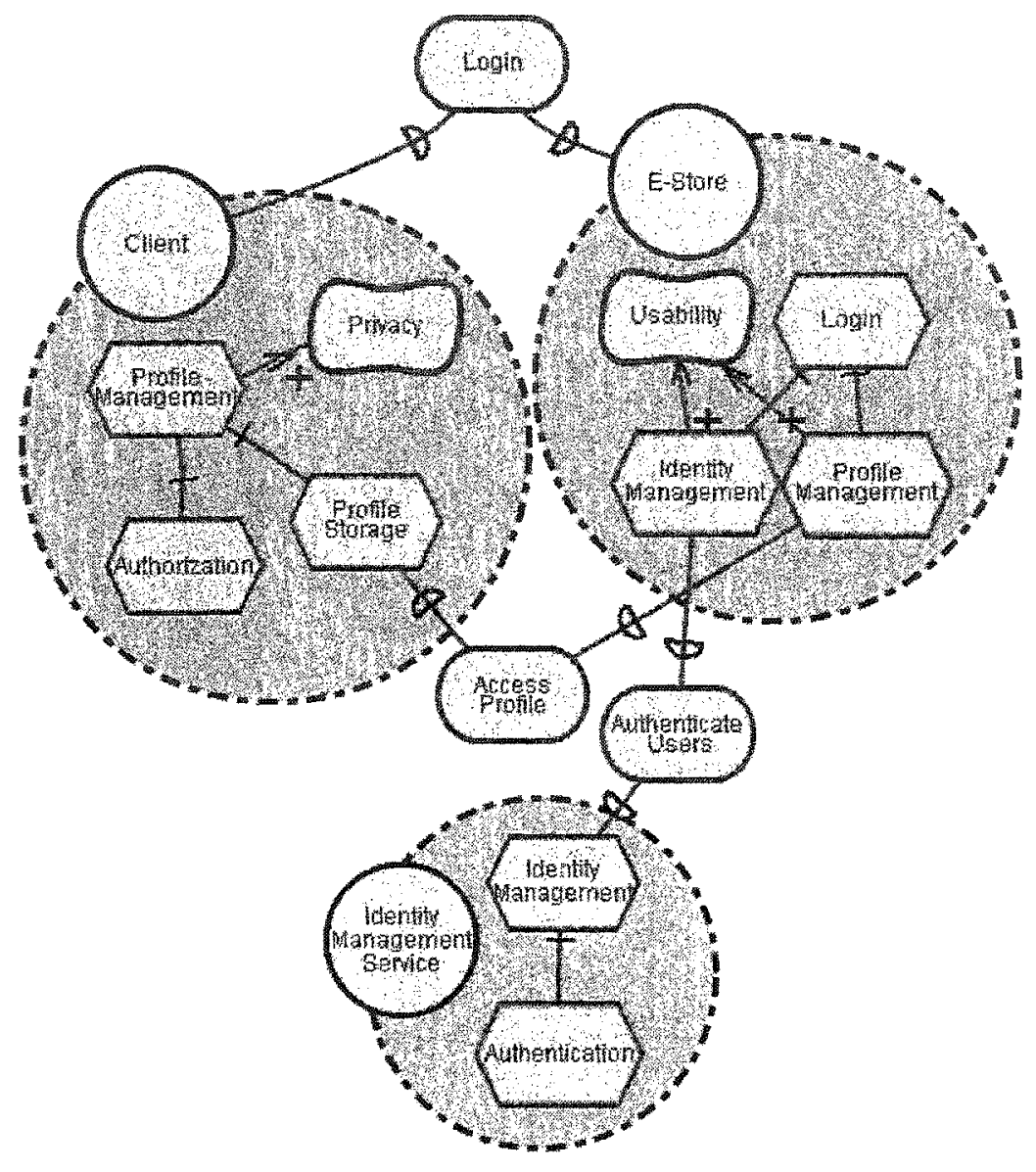

Figure 5.17: Decouple the Authentication and Authorization Features to Avoid the Feature Interaction

\section{Step 7: Document the Feature Interaction}

Document 5.1:

Name: Usability vs. Privacy

Feature Interaction Category: NFEMR (NF-feature interaction is non-functional.

E-feature interaction occurs in two actors: Client and E-Store. MR-E-Store provides services to client and consumes service from NPS. Therefore, it acts in two 
roles.)

Cause: Goal Conflicts (see §4.2.5) and Deployment and Ownership (see §4.2.6)

Behavior: $\log$ in 0

Web Service Application Layer: Presentation Layer

\subsubsection{Search \& Display Function}

\section{Step 1: Analyze Problem Descriptions}

The scenario for this function is the following:

- The user is authenticated by the e-store system. Then he/she can search the products by keyword.

- The user inputs the text of the item to be searched and clicks the "search" button.

- The e-store display service will search local catalog. If it cannot find the item, it will automatically invoke Boogle Search (BS) for searching the same item. The e-store will then call Remote Search $(R S)$ in the Remote Search Service(RSS).

\section{Step 2: Model Features as Goal Graphs}

\section{- Features}

- Local Search

- Boogle Search

- Remote Search 


\section{Non-functional Concerns}

- Performance

Two aspects relate to performance. One is the minimum amount of time needed to process a given task. The other is maintaining the amount of time needed for each task if the number of requests for that task increases [IBM03]. It includes two attributes [Araujo02]:

- Response time: Amount of time required to respond to an event.

- Latency: Time elapsed between a request and a response.

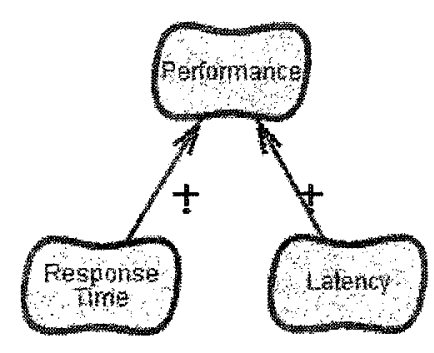

Figure 5.18: Force Hierarchy for Performance

- Maintainability

Maintainability can be measured by side effects in changing code and code modifiability. Minimizing the side effects in changing code can improve the maintainability [Araujo02].

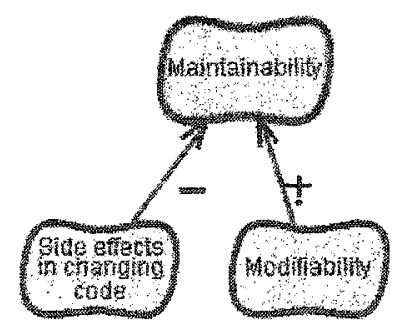

Figure 5.19: Force Hierarchy for Maintainability 
- Interoperability

Interoperability aims to offer seamless and automatic connections among Web services. Web services interoperability problem exists in three ways: discovery, definition, and request/response mechanisms [Cohen02].

\section{- Discovery}

Interoperability problems exist at the discovery because the current UDDI allows multiple taxonomies. Hence, errors and confusion may exist inside the directory structures in UDDI. Those mistakes will not be corrected by the current UDDI self -policing mechanisms.

- Definition

WSDL is used to define how to make a request to a SOAP-based method in Web services. However, without specifying the namespace in the WSDL documents, data types mismatch may result in interoperability problems.

- Request/Response mechanisms

Developers write the SOAP requests in their own way. Many SOAP tools add explicit data types information into the request and response document when the related element is deserialized. However, some SOAP libraries return response without type information. This may result in interoperability problems. 


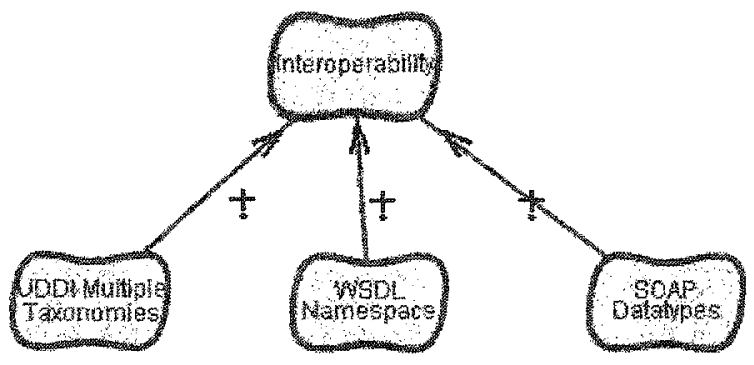

Figure 5.20: Force Hierarchy for Interoperability

\section{Step 3: Describe Scenarios by UCM}

In this scenario, Local Search (LS) in the Local Search Service (LSS) will search the local catalog. If no result can be found, it will invoke Boogle Search $(B S)$ in the Boogle Search Service (BSS). Then the e-store will call Remote Search (RS) in the Remote Search Service (RSS).

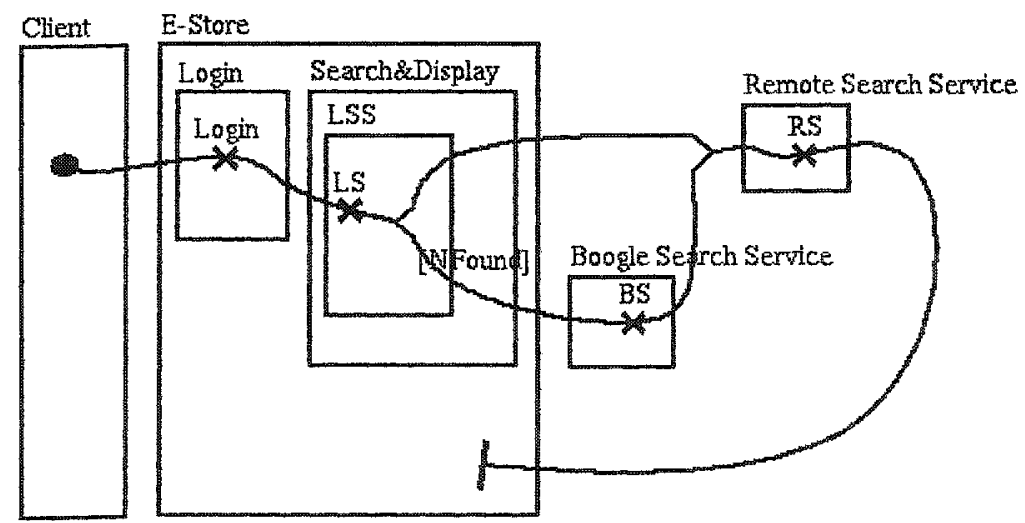

Figure 5.21: Use Case Map for the Search \& Display Function

Notes for Figure 5.21:

LSS - Local Search Service; LS - Local Search; BS-Boogle Search; RS- Remote Search

\section{Step 4: Explore Feature Interactions}


Example 5.2: Interoperability vs. Maintainability

As the above scenario shows, this function is performed by two features $L S$ and $R S$. These two features have been provided by two services. Therefore, changing one feature will not affect another one. That improves the maintainability of the e-store system. Once the $B S S$ receives the user's request, it will pass the query request to the marketplace, which will redirect the request to the supplier using a new SOAP service request. However, the supplier is only able to respond to catalog queries using another vocabulary set, such as the RosettaNet [RosettaNet03] vocabulary, which is different from the vocabulary set of the LSS. This means that $B S$ returns the results with unexpected vocabulary to the Search \& Display function. This may result in the wrong display. This means it reduces the interoperability of the e-store system. Hence, we can say there is a feature interaction between Interoperability and Maintainability (see Figure 5.22).

\section{Example 5.3: Maintainability vs. Performance}

Another issue is that the BSS will be invoked twice if the RSS is the BSS. This will degrade the e-store performance. Hence, we can say that there is a feature interaction between Maintainability and Performance (also see Figure 5.22). 


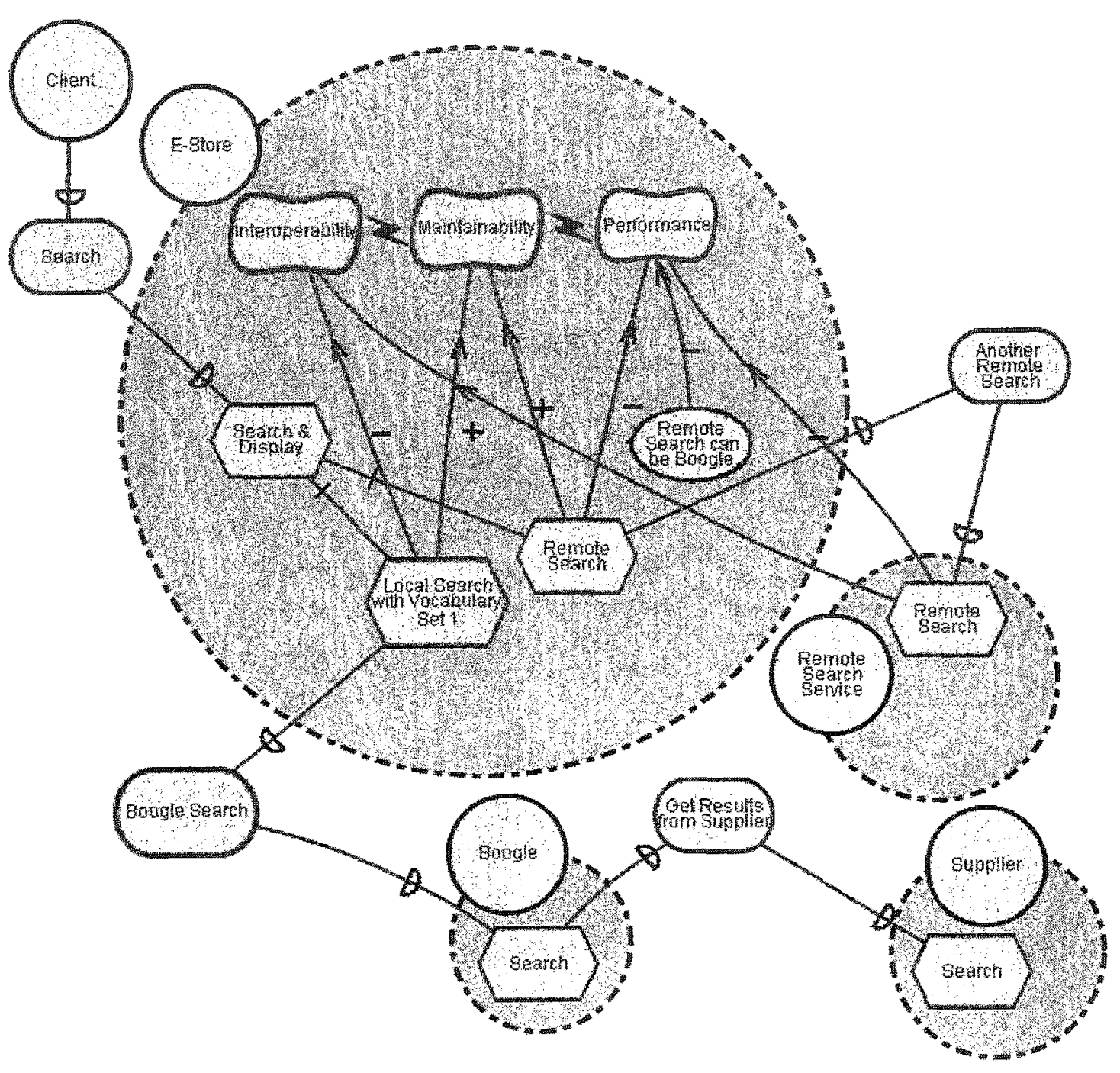

Figure 5.22: Feature Interactions among Interoperability, Maintainability, and

Performance

\section{Step 5: Using UCM to Analyze Deployment Issues}

As the above analysis shows, there is no deployment issue in Example 5.2. In

Example 5.3, as shown in the following figure, if RSS is BSS, BSS may be invoked twice.

This is a deployment issue. 


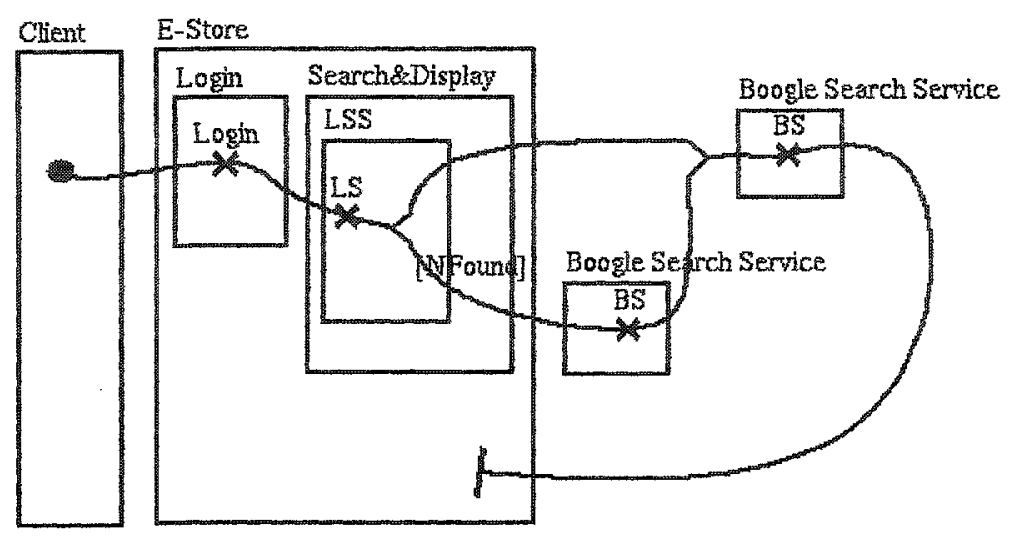

Figure 5.23: Using Use Case Map to Analyze Deployment Issue Notes for Figure 5.23:

LSS-Local Search Service; LS-Local Search; BS-Boogle Search

\section{Step 6: Solution and Discussion}

In Example 5.2 and Example 5.3, the feature interactions are non-functional. They occur in an actor: E-Store. E-Store acts as a service provider and offers the service to the client. It also invokes the BSS. Therefore, the feature interactions are NFIMR feature interactions. In Example 5.2, LSS and the supplier of BSS use the different vocabulary sets. In other words, LSS violates the vocabulary set assumption of the supplier of BSS. Therefore, for this particular example, Violation of Assumptions about Service (see \$4.2.1) will be a cause of feature interaction. Conflicts occur between non-functional concerns: Interoperability and Maintainability. This affects the user's total satisfaction. Meanwhile, it proves the current solution produces unanticipated side effects. Therefore, Goal Conflicts (see \$4.2.5) will be another cause of this feature interaction. In Example 5.3, the reason of the interaction is that BSS can be deployed as a RSS. Therefore, Deployment and Ownership (see $\$ 4.2 .6$ ) is the cause of this feature interaction. The 
current solution improves the maintainability. However, it may result in unanticipated performance problem. Therefore, Goal Conflicts (see $\$ 4.2 .5$ ) can be another cause of this feature interaction.

The solution for Example 5.2 is to add a translation service to map the vocabulary sets between $B S S$ and the supplier when $B S S$ redirects the customer's query to the supplier. If $B S S$ 's vocabulary set matches the supplier's, $B S S$ will redirect the request to the suppliers with the same vocabulary set.

The solution for Example $\mathbf{5 . 3}$ is to add a function to check whether a particular RSS has been invoked before, if yes, the e-store system will eliminate it. In this example, the e-store system will use a function to check whether $B S S$ has been invoked before.

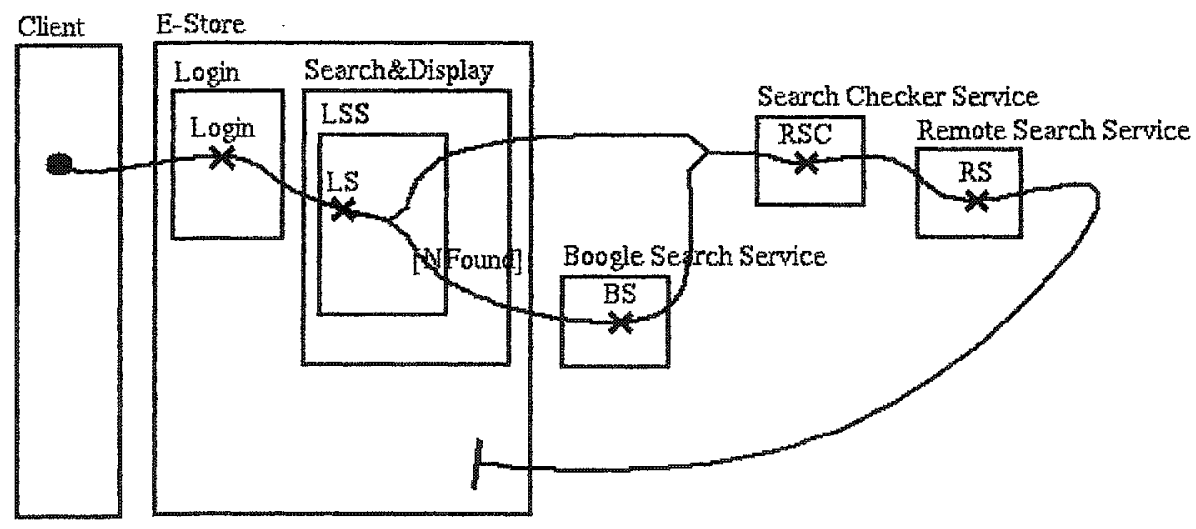

Figure 5.24: Refined the Use Case Map

Notes for Figure 5.24:

LSS-Local Search Service; LS-Local Search; BS-Boogle Search; RSS-Remote Search Service; RS-Remote Search 


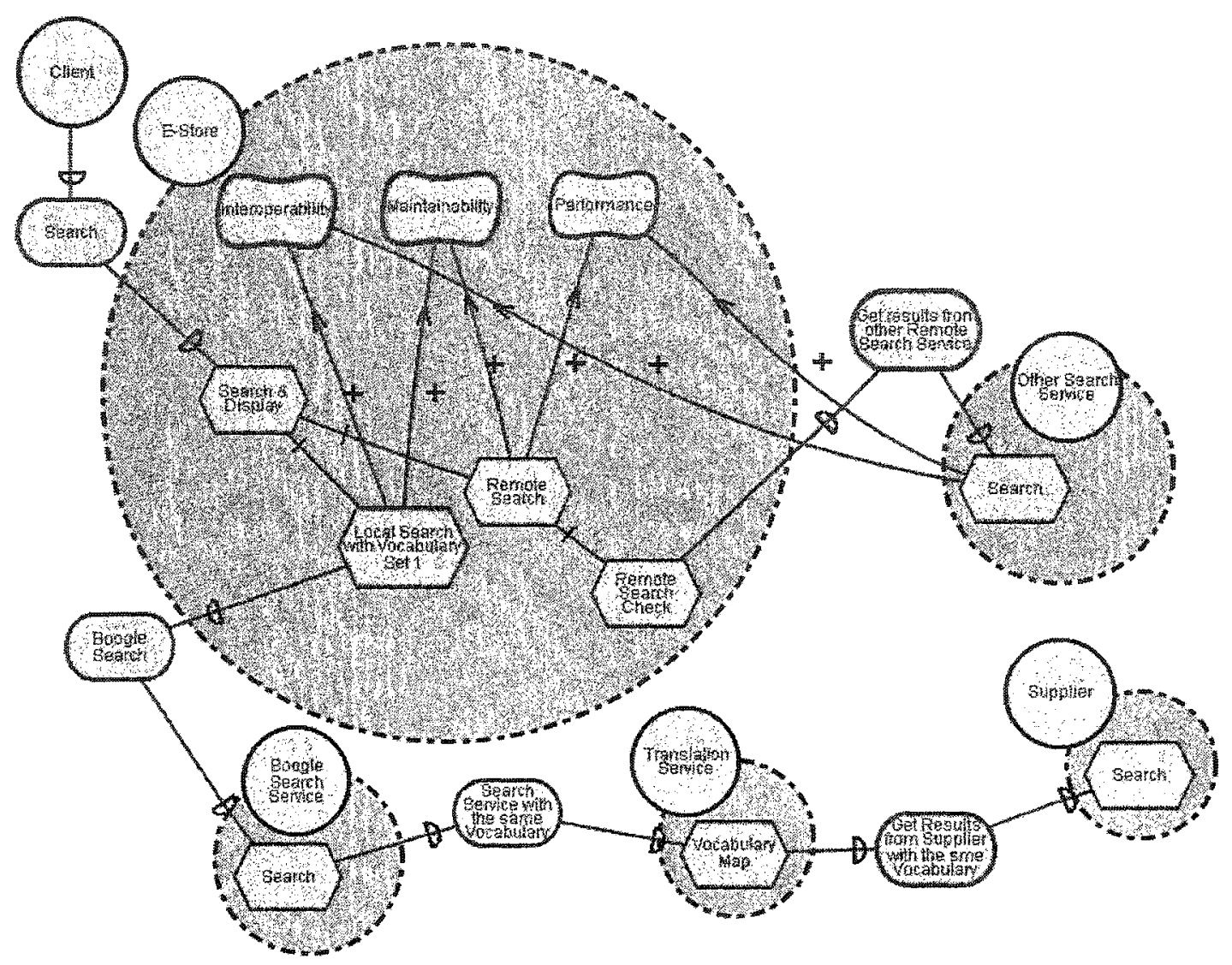

Figure 5.25: Using Vocabulary Map and Remote Search Check to Avoid the Feature Interaction

This solution improves the interoperability of the e-store system by using the same vocabulary set. It also improves the performance by avoiding invocation of the $B S S$ twice. However, it increases the complexity of the system by importing a new search checker service. This may reduce the performance of the e-store system.

\section{Step 7: Document the Feature Interaction}

Document 5.2:

Name: Manageability vs. Interoperability 
Teature Interaction Category: NFIMR (NF-feature interaction is non-functional. Ifeature interaction occurs in a single actor: E-Store. MR-E-Store provides services to client and consumes BSS. Therefore, it acts in two roles.)

Cause: Violation of Assumptions about Service (see \$4.2.1) and Goal Conflicts (see

Behavior: Boogle Search

Web Service Application Layer: Data Layer

Document 5.3:

Name: Manageability vs. Performance

Feature Interaction Category: NFIMR (NF-feature interaction is non-functional.

I-feature interaction occurs in a single actor: E-Store. MR-E-Store provides services to client and consumes BSS. Therefore, it acts in two roles.)

Cause: Deployment and Ownership (see $\$ 4.2 .6$ ) and Goal Conflicts (see $\S 4.2 .5$ )

Behavior: Remote Search

Web Service Application Layer: Data Layer

\subsubsection{Order Processing Function}

\section{Step 1: Analyze Problem Descriptions}

The scenario is the following:

- After the user finds the expected items, he/she can place orders.

- He/she will enter the quantities of expected items and click "Place an Order" button. 
- After the user clicks the button, the e-store system will check the quantities the user entered.

The e-store system will search the local catalog and place an order for those items.

- If the quantities of items in the local catalog are less than the amount the user entered, the e-store system will automatically invoke the Amazin Web Service (AWS) to place an order for them. If the quantities are not reached, the AWS will find another Web service to place an order until the quantities are satisfied.

\section{Step 2: Model Features as Goal Graphs}

Features

- Places a Local Order

- Places an Order on Amazin

- Places a Remote Order

- Non-Functional Concerns

- Maintainability

- Performance

\section{Step 3: Describe Scenarios by UCM}

In the following UCM model, there are three main components: Local Order Process

Service (LOPS), AWS, and Remote Order Process Service (ROPS). Every component 
includes several functions, such as to check item quantities, and to place an order. When a valid user wants to order items, first, he/she needs to enter item quantities. The local order process service will check the local quantities, and if there are enough items to satisfy the order, it will place a local order. Otherwise, it will calculate the additional quantities, and place an order to $A W S$. If there are not enough quantities to satisfy the order, $A W S$ will call other ROPS to place an order.

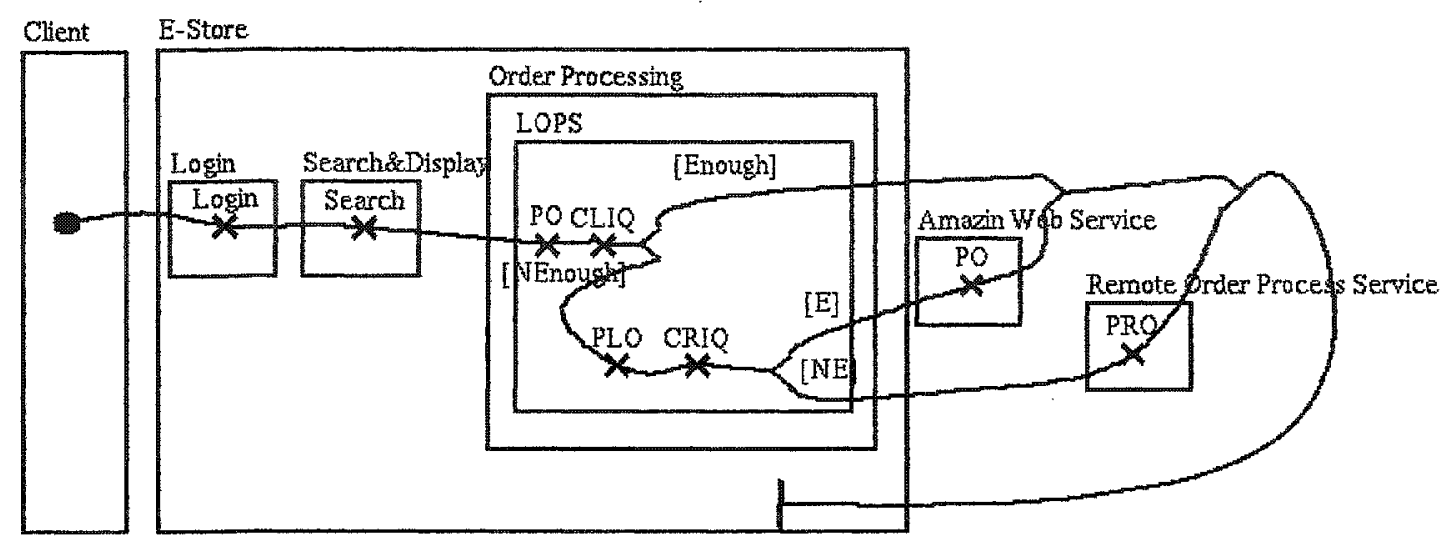

Figure 5.26: Use Case Map for the Order Processing Function

Notes for Figure 5.26:

LOPS-Local Order Process Service; PO- Process Order; CLIQ-Check Local Item Quantities;

PLO Place Local Order; CRIQ - Check Remote Item Quantities; PRO-Place Remote Order

\section{Step 4: Explore Feature Interactions}

\section{Example 5.4: Maintainability vs. Performance}

As the above scenario shows, when the quantities of the items in the local catalog are less than the ones the user enters, the e-store system will invoke the $A W S$ automatically. This reduces human involvement. These features have been provided by separate services. Therefore, changing one feature will not affect another one. These improve feature's maintainability by maintaining features separately and 
reducing human involvement. However, if the $A W S$ also does not have enough items in stock to fulfill this order, it will automatically search other catalogs on the Internet, and it is very possible that it will forward the request back to the LOPS. Therefore, the LOPS and the AWS will form an infinite loop to make request, and this will use up the system memory resource of the server hosted the e-store system, and eventually, the user will not get response from the e-store system. Therefore, we can say that there is a feature interaction between feature maintainability and performance.

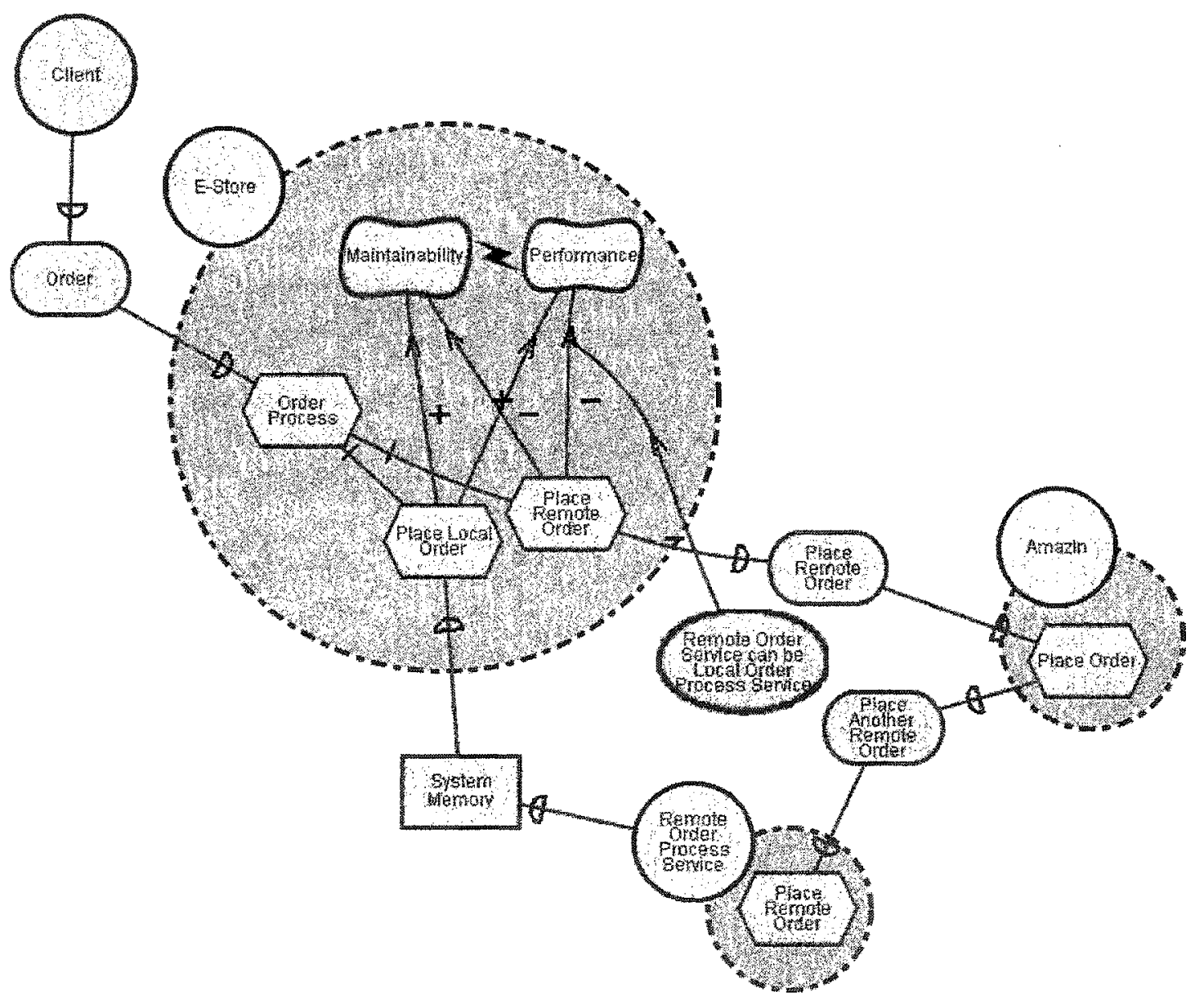

Figure 5.27: Order Processing Produces Infinite Loops and Reduces the Performance 


\section{Step 5: Using UCM to Analyze Deployment Issues}

As shown in Figure 5.27, although PLO and PRO are located in different components, it is possible that ROPS can be the LOPS in another e-store system. In this case, PRO can be PLO. Then, in Example 5.4, LOPS and AWS could be invoked for many times. This is a deployment issue.

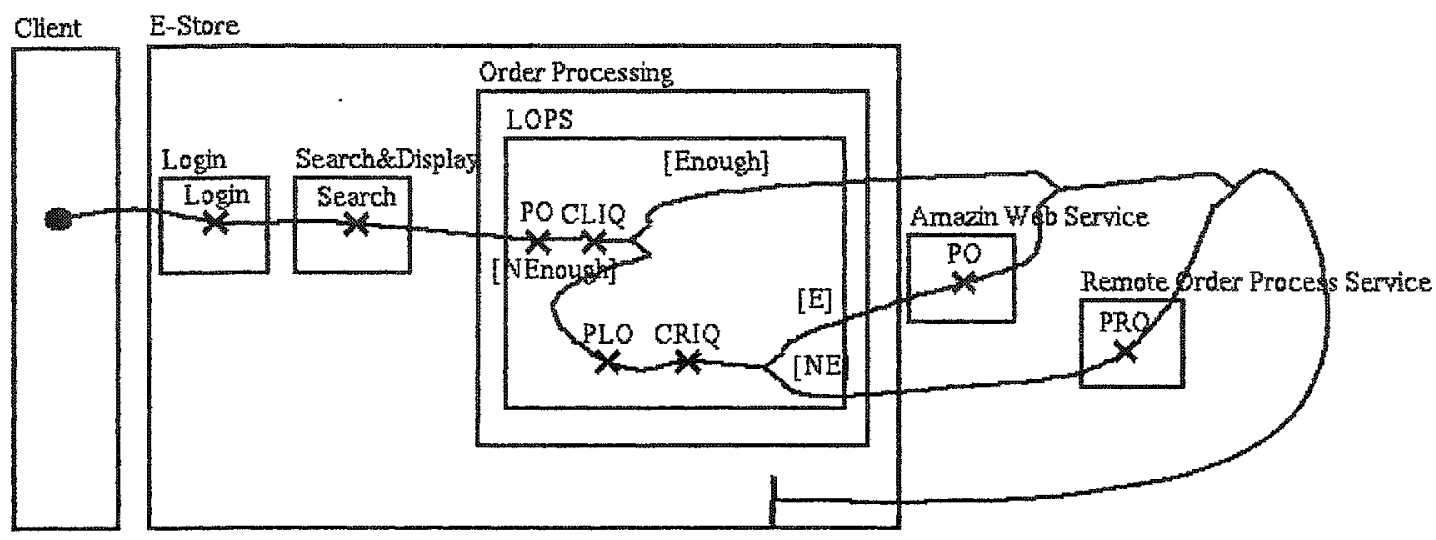

Figure 5.28: Using UCM to Analyze the Deployment Issue

\section{Notes for Figure 5.28:}

LOPS - Local Order Process Service; PO - Process Order; CLIQ Check Local Item Quantities; PLO - Place Local Order; CRIQ_ Check Remote Item Quantities; E-Enough; NE-Not Enough; PRO-Place Remote Order

\section{Step 6: Solution and Discussion}

In this example, the feature interaction occurs between non-functional concerns. It occurs in a single actor: E-Store. E-Store acts in multiple roles by providing LOPS for client and consuming the $A W S$. Therefore, it is a NFIMR feature interaction. As we analyzed above, automatically order processing produces infinite loops. This will use up the system memory resource and result in a serious performance problem. Therefore, Resource Contention will be a cause of this feature interaction. The solution for the e- 
store order processing produces the unanticipated side effects while it improves the maintainability. Therefore, Goal Conflicts can be another cause of this feature interaction.

\section{An intuitive method is to add a Remote Service Checker (RSC) in Remote Order} Process Checker Service (ROPCS) before invoking the ROPS. This checker can prevent the ROPS from invoking the LOPS or itself. The remote service check function will check the information, which is defined by each service provider. For example, each service provider may provide a maximum number of order requests. This function will check if the order number has reached the maximum value, and it will stop service if the maximum value has been reached. This can avoid infinite loops. Each service provider can also save the recent service requests history and service providers history into a request user list. The check function can check the list and send an alert to the service provider to confirm the order if it finds the request exists in the recent request user list and if the service provider was previously involved. The refined UCM and GRL are shown in Figure 5.29 and Figure 5.30 respectively.

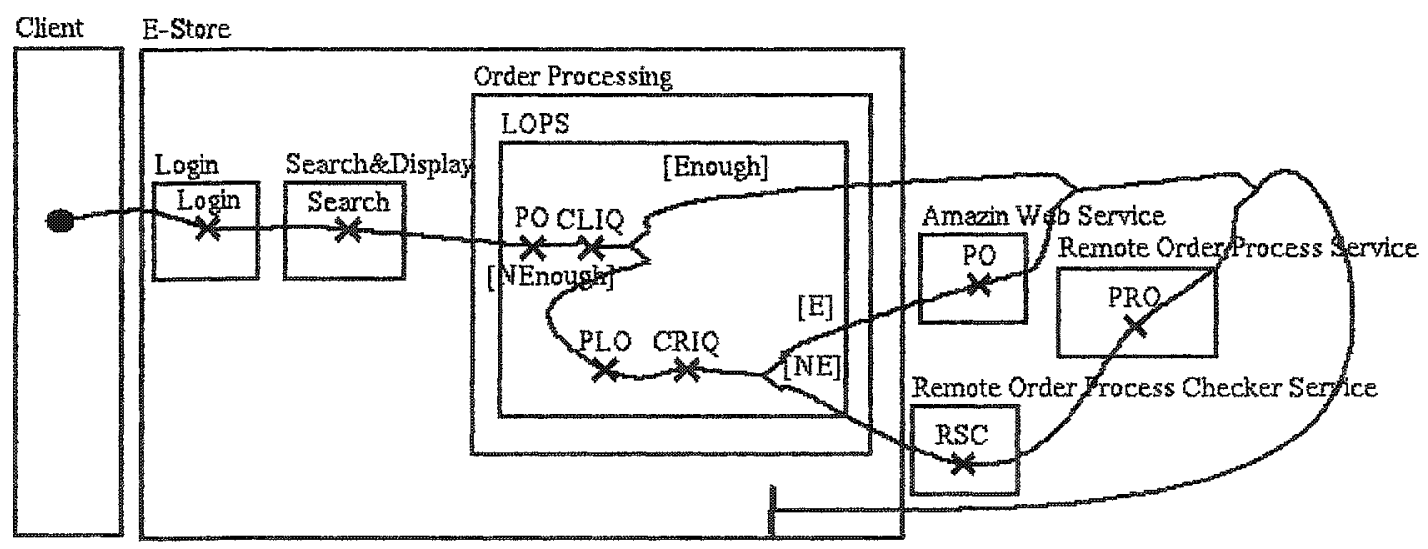

Figure 5.29: Refined Use Case Map

Notes for Figure 5.29:

LOPS Local Order Process Service; PO-Process Order; CLIQ-Check Local Item Quantities; PLO-Place Local Order; CRIQ-Check Remote Item Quantities; E-Enough; NE-Not Enough; 
RSC-Remote Service Checker; PRO-Place Remote Order

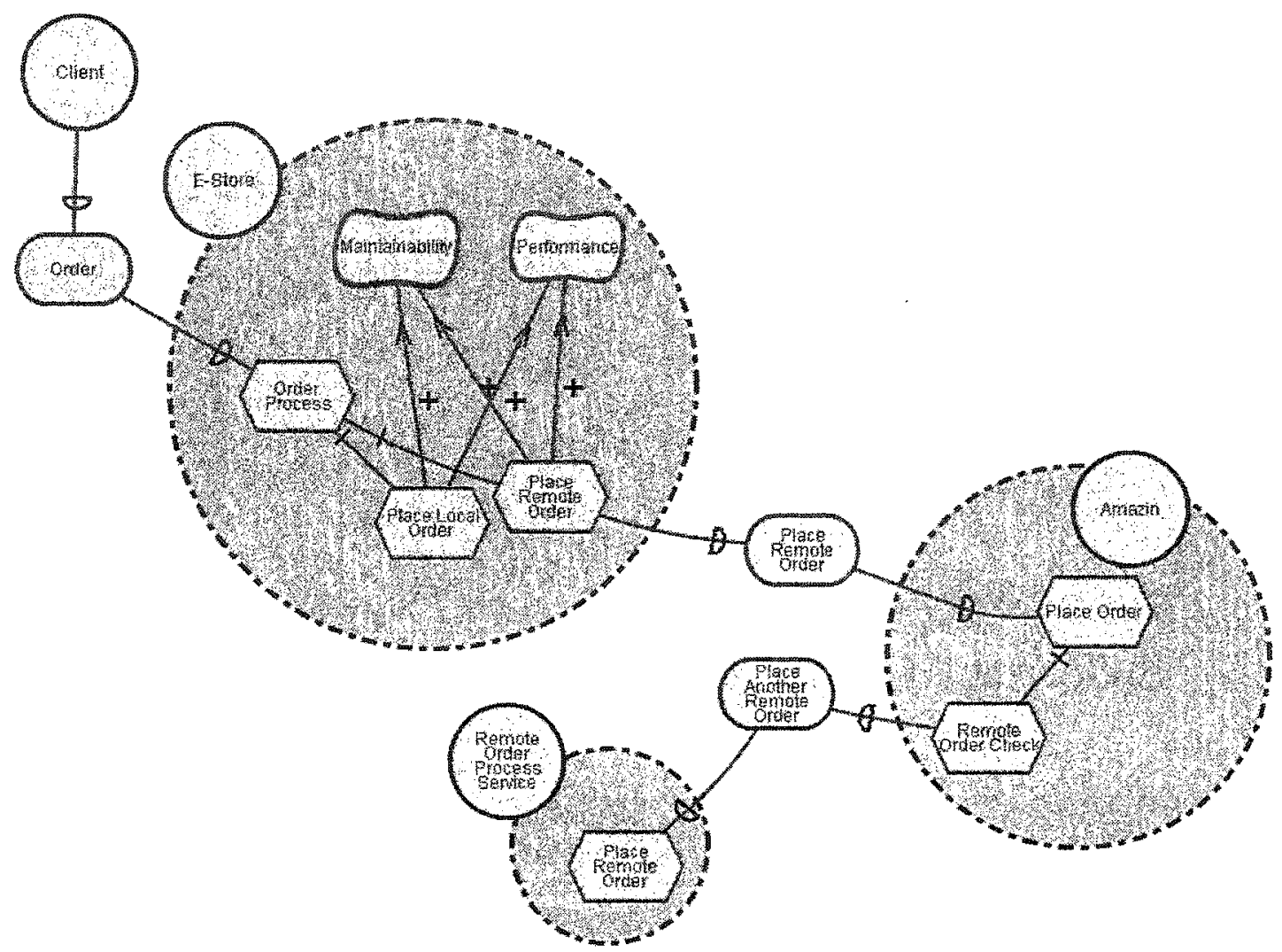

Figure 5.30: The Solution for Feature Interaction

This solution avoids the infinite loops among the different order process services by adding the additional service provider checker. This operation adds additional overhead. Therefore, it degrades the efficiency a little. However, that won't introduce a new feature interaction.

\section{Step 7: Document the Feature Interaction}

Document 5.4:

Name: Maintainability vs. Performance 
Feature Interaction Category: NFIMR (NF-feature interaction is non-functional.

I-feature interaction occurs in a single actor: $E$-Store. $M R-E$-Store provides services to client and consumes Amazin. Therefore, it acts in two roles.)

Cause: Resource Contention (see \$4.2.4) and Goal Conflicts (see §4.2.5)

Behavior: Place Remote Order

Web Service Application Layer: Logic Layer

\subsubsection{Billing \& Shipping Function}

\section{Step 1: Analyze Problem Descriptions}

The scenario is the following:

- The user makes orders for the products, and clicks "checkout" button

- The e-store system checks the discount type for the user

- The e-store system calculates the cost for those items

- The e-store asks the user to choose a shipping method

- The e-store system will calculate the tax for items and the chosen shipping method

- The e-store system will ask the user to enter credit card number and shipping address

- After the e-store finishes processing the order, it will send an order confirmation to the user's mailbox 


\section{Step 2: Model Features as Goal Graphs}

\section{Features}

- Group Discount

- Member Discount

- Shipping by UPS

The e-store system will not calculate the items' tax if the user chooses shipping by UPS.

- Shipping by Mail

The e-store system will calculate GST and PST for the items if the user chooses shipping by Mail.

- Tax Calculation

\section{* Non-Functional Concerns}

\section{- Profitability}

This feature means the ability to earn a profit. In the e-store system, users can earn profit from the different discount policies. The e-store can also increase volume at lower price. This means the e-store may get larger revenue.

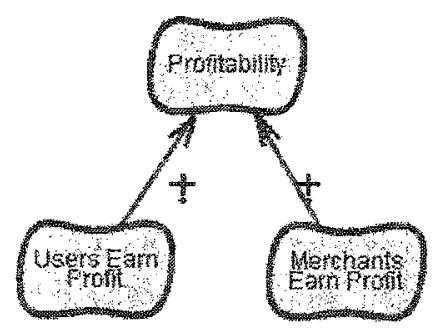

Figure 5.31: Force Hierarchy for Profitability 
- Correctness

Web services correctness means that Web services behave as intended. This includes two situations. One is that Web services cannot get the expected result; the other is that Web services cannot make a choice which of multiple results should be returned.

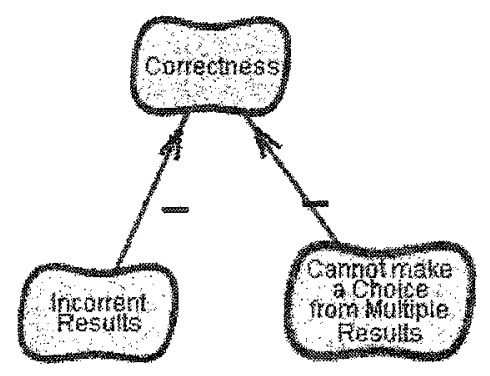

Figure 5.32: Force Hierarchy for Correctness

\section{Step 3: Describe Scenarios by UCM}

In this UCM model, there are four components involved in this function: Billing \& Shipping, Payment Discount Service, Shipping Service, and Tax Service. When the valid user clicks "checkout" button, the e-store will check the discount policy applicable to the user by Check Discount Policies (CDP).

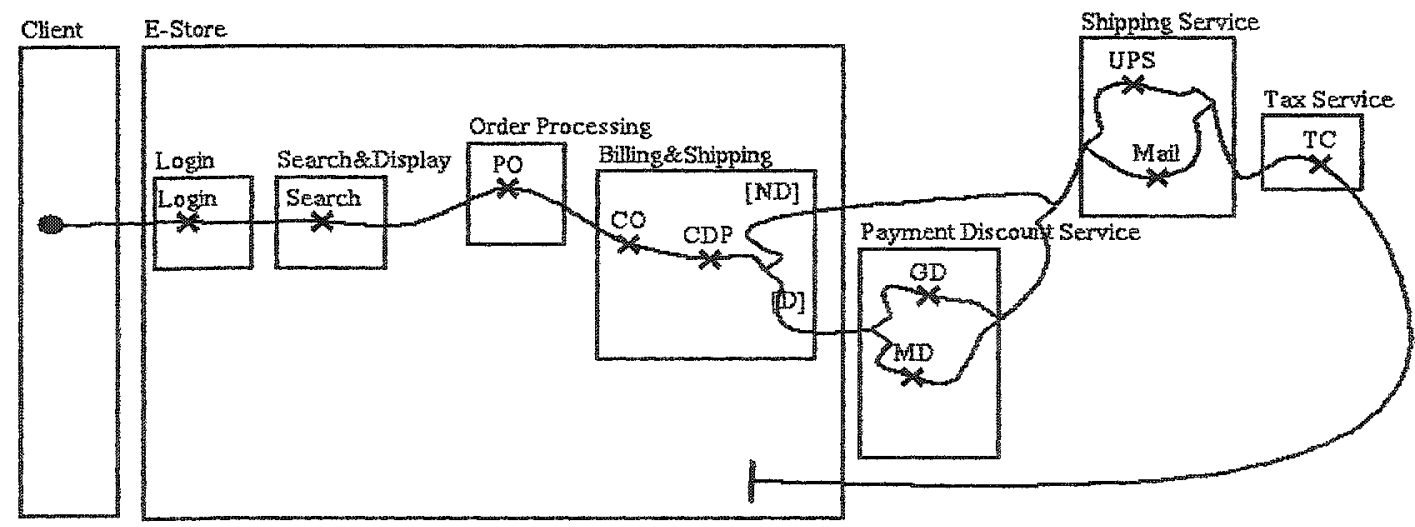

Figure 5.33: Use Case Map for the Billing \& Shipping Function 
Notes for igure 5.33:

PO-Process Order; CO-Check Out; CDP_-Check Discount Policies; GD-Group Discount; MDMember Discount; TC-Tax Calculation; UPS - UPS delivery; Mail-Mail delivery

\section{Step 4: Explore Feature Interactions}

\section{Example 5.5: Profitability vs. Correctness}

The e-store system offers different discount types for different users. However, it only offers one single discount type for one user. These discount types can be made for different purposes. We can separate them into three types by the people who will benefit from: customer, merchant, and customer and merchant. In every category, we assume to classify two types: group discount and member discount, which serve the users with group membership and VIP membership respectively. This improves the flexibility of the e-store system and increases the volume at lower price. Furthermore, it may also attract and encourage customers to access the service again. Hence, we can say different discount policies can improve this user's and the e-store's profitability.

If the user belongs to either a group or a VIP membership, his discount type will be either member or group discount type, these discount policies will not conflict with each other. However, if the user belongs to both group membership and VIP membership, his discount type is both group discount and member discount, then the e-store system will not make a choice from those two discount types for the user. This breaks the correctness of the e-store system. Hence, different discount policies improve user's profitability, while it also breaks the correctness of the e-store system (see Figure 5.34). 


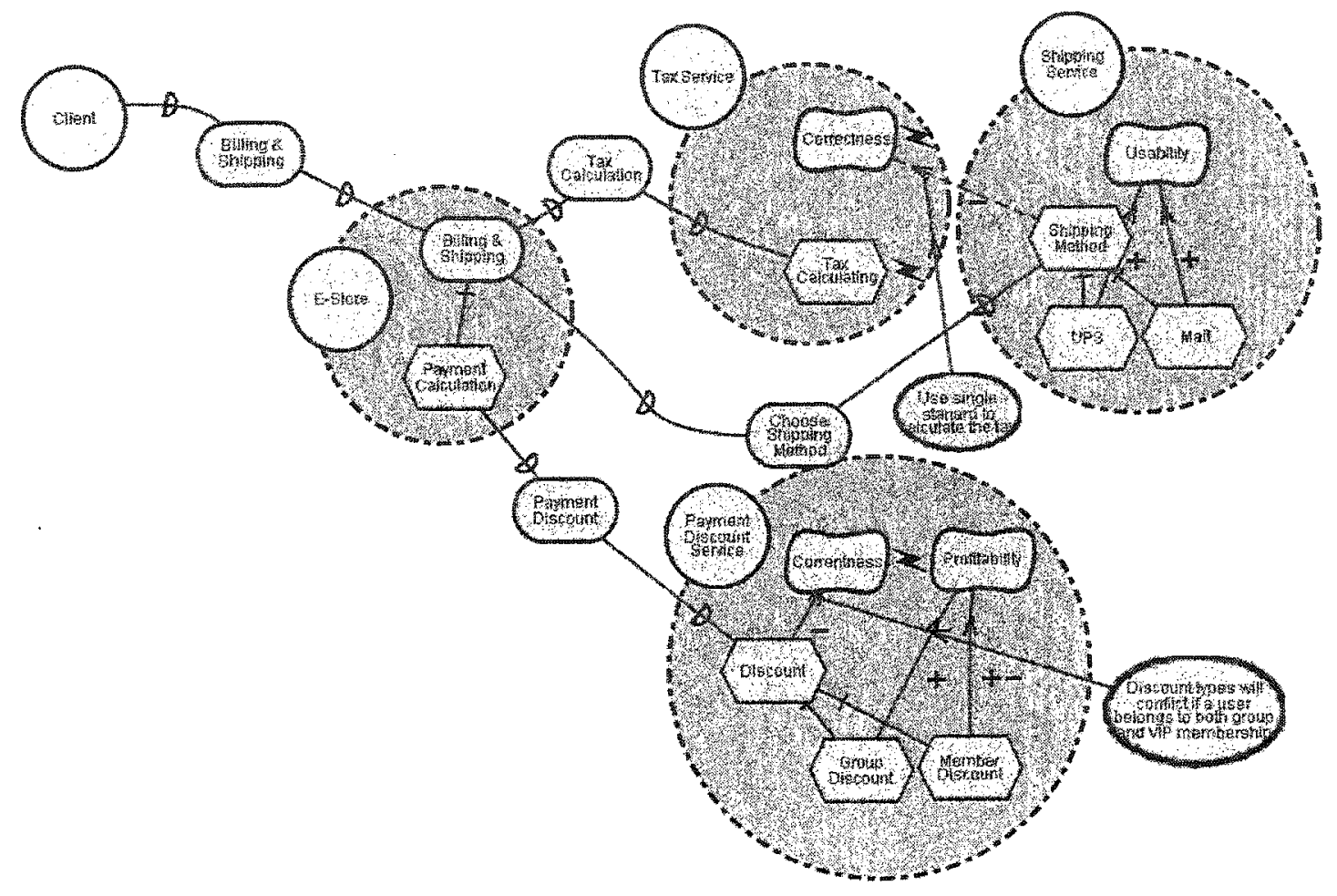

Figure 5.34: Feature Interactions in Billing \& Shipping Function

Example 5.6: Tax Calculating vs. Shipping Method

The e-store system uses different tax calculation methods for different shipping methods. For example, if the user chooses the shipping method UPS, the e-store will not calculate the tax. If the user chooses Mail to delivery items, the e-store will calculate GST and PST for the items. If the user chooses multiple items with multiple shipping methods, the TS should have applied the different tax calculation method for those items. However, the TS is a third party service with a single standard to calculate the tax. It will calculate GST and PST for all items regardless of the shipping methods. Hence, in this case, there is feature interaction between feature Tax Calculating and Shipping Method (also see Figure 5.34). 
Example 5.7: Correctness vs. Usability

As the above scenario shows, different shipping methods provide flexible shipping choices for the user. It can attract the user to be back to use the service again. It improves the usability of the service by improving the service flexibility. However, it defeats the correctness of the TS. Therefore, interaction also occurs between non-functional concerns: usability and correctness (also see Figure 5.34).

Example 5.8: Correctness vs. Usability

We make a few changes for the above scenario. As shown in the following figure, we assume the e-store need to display different currencies according to different users in different counties by using TS to invoke a Currency Exchange Service (CES) to perform currency exchange. In this scenario, the feature interactions in Example 5.6 will occur in multiple roles because $T S$ acts in two roles in this scenario: service provider and service consumer. It provides the service to the e-store system as a service provider, and it acts as a service consumer to consume $C E S$. 


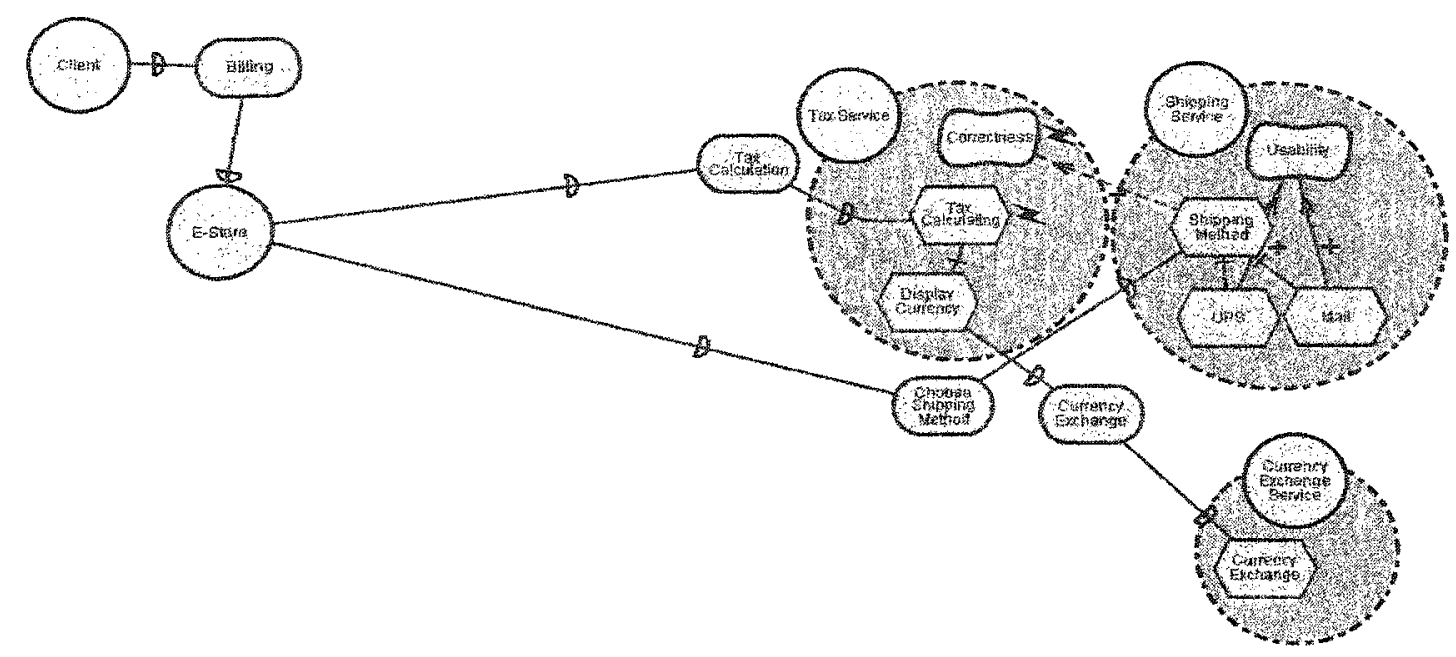

Figure 5.35: Another Feature Interaction in Billing \& Shipping Function

\section{Step 5: Using UCM to Analyze Deployment Issues}

As the above analysis shows, no deployment issue exists in this scenario.

\section{Step 6: Solution and Discussion}

In Example 5.5, the feature interaction occurs between two non-functional concerns.

It occurs in a single actor: Payment Calculation Service, which acts as a service provider. Therefore, this feature interaction is a NFISR feature interaction. The e-store system cannot make a choice in multiple discount policies. Therefore, Policy Conflict is one of the causes of this interaction. The solution improves the profitability, while it reduces correctness. Therefore, Goal Conflicts is another cause.

In $\mathrm{Lxample}$ 5.6, the feature interaction occurs between two functional requirements. It occurs in two actors: $T S$ and $S S$, which both act as a service provider. Therefore, this feature interaction is a FESR feature interaction. The e-store violates the assumption of Tax Service. Therefore, Violation of Assumptions about Service is the cause. 
In Example 5.7, the feature interaction occurs between two non-functional concerns. It occurs in two actors: $T S$ and $S S$, which both act as a service provider. Therefore, this feature interaction is a NFESR feature interaction. Different shipping choices improve the usability of the service, but it defeats the correctness of the service. Therefore, Violation of Assumptions about Service and Goal Conflicts are causes.

In Example 5.8, the feature interaction occurs between two non-functional concerns. It occurs in two actors: TS and SS. The Tax Service acts as two roles: service provider and service consumer. Therefore, this feature interaction is a NFEMR feature interaction. The causes for this example are same as the Example 5.7.

As the above analysis shows, the solution for Example 5.5 is to use a function called discount adjustment to avoid the feature interaction. This function will use a new policy to adjust the discount types. This new policy can be made in many kinds of ways depending on who benefit from it. In this case, we assume the policy benefits the user. Therefore, we may make the policy like this:

If a customer can get both a member discount and a group discount, because the member discount gives more discount than group discount, so the discount type for the customer will be the member discount.

The solution for Example 5.6, Example 5.7, and Example 5.8 is to use a shipping method check in the TS to check the shipping method before the TS calculates the tax for items. The following figure is the refined GRL model. 


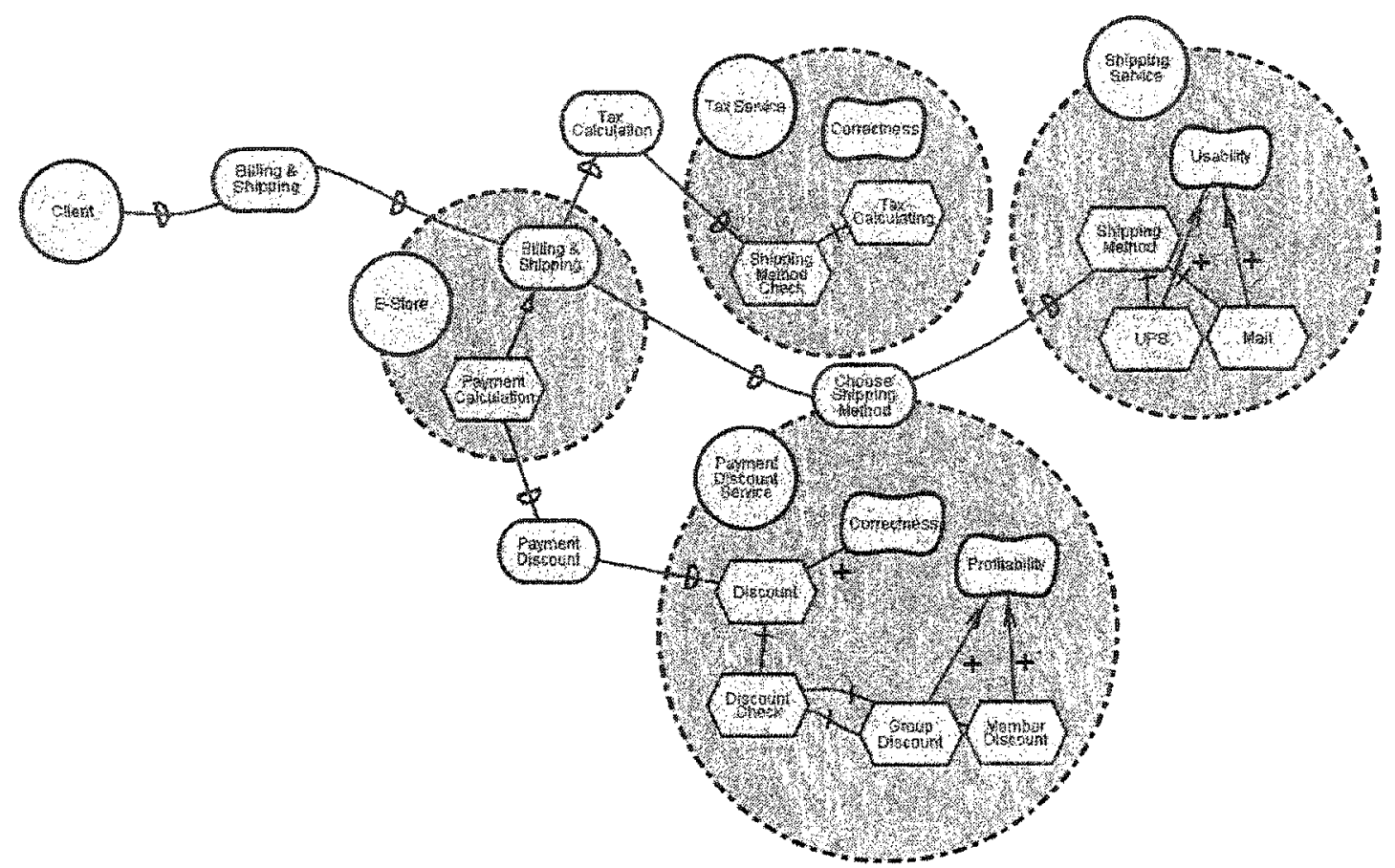

Figure 5.36: Applying the new Policy can Avoid the Feature Interaction

The following UCM model is a refined model for this scenario. In this model, we add a Discount Check (DC) to check the discount type. This function is located in the PDS. We also add a Shipping Check (SC) into the component TS.

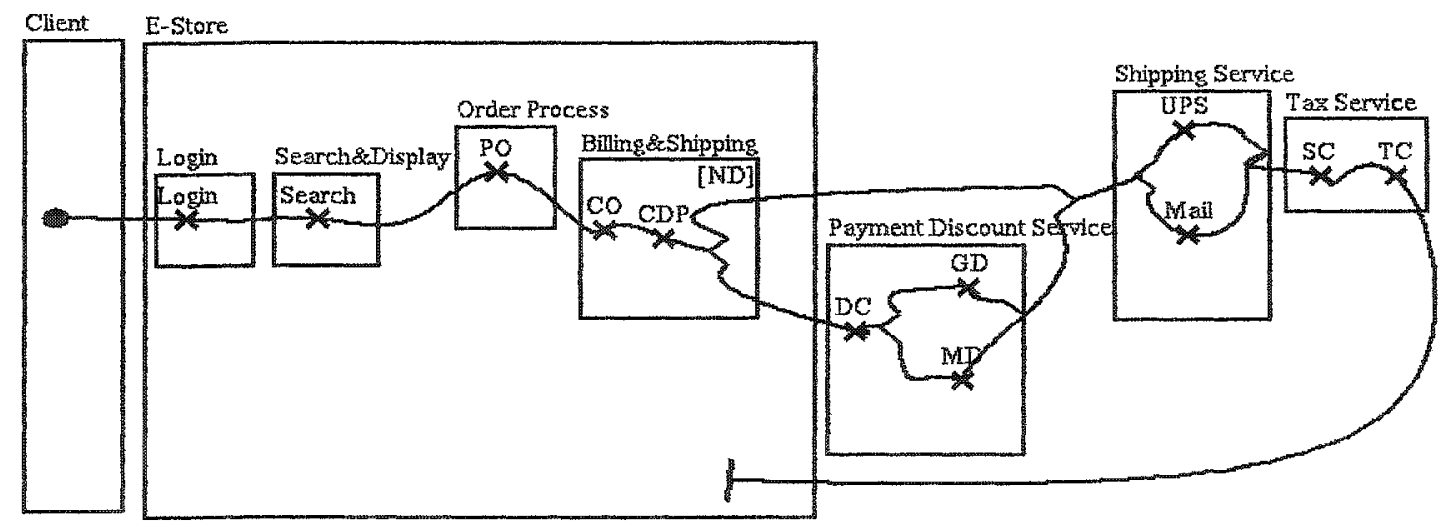

Figure 5.37: Refined Use Case Map for the Billing \& Shipping Function

\section{Notes for Figure 5.37:}

PO Process Order; CO-Check Out; CDP-Check Discount Policies; DC - Discount Check; GDGroup Discount; MD-Member Discount; SC - Shipping Check; TC-Tax Calculation 
This solution improves service correctness by adjusting the discount type. Meanwhile, it also adds the complexity of the service. In some way, it degrades the performance of the service.

\section{Step 7: Document the Feature Interaction}

Document 5.5:

Name: Profitability vs. Correctness

Feature Interaction Category: NFISR (NF-feature interaction is non-functional. I-

feature interaction occurs in a single actor: Payment Calculation Service (PCS). SRPCS provides service to E-Store as a service provider.)

Cause: Goal Conflicts (see \$4.2.5) and Policy Conflict (see §4.2.4)

Behavior: Discount Calculation

Web Service Application Layer: Logic Layer

Document 5.6:

Name: TaxCalculating vs. Shipping Method

Feature Interaction Category: FESR ( $F$-feature interaction is functional. $E$-feature interaction occurs between two actors: TS and SS. SR-both TS and SS provide service to E-Store as service providers.)

Cause: Violation of Assumptions about Service (see \$4.2.1)

Behavior: Tax Calculation

Web Service Application Layer: Logic Layer 
Document 5.7:

Name: Correctness vs. Usability

Feature Interaction Category: NFESR NF-two non-functional concerns:

correctness and usability. E-feature interaction occurs between two actors: TS and

SS. SR - both TS and SS provides service to E-Store as service providers.)

Cause: Violation of Assumptions about Service (see \$4.2.1) and Goal Conflicts (see $\$ 4.2 .5)$

Behavior: Tax Calculation

Web Service Application Layer: Logic Layer

Document 5.8:

Name: Correctness vs. Usability

Feature Interaction Category: NFEMR (NF-two non-functional concerns:

correctness and usability. E-feature interaction occurs between two actors: TS and SS. MR-TS provides service to E-Store and consumes CES. Therefore, it acts in two roles.)

Cause: Violation of Assumptions about Service (see \$4.2.1) and Goal Conflicts $(\operatorname{see} \$ 4.2 .5)$

Behavior: Tax Calculation

Web Service Application Layer: Logic Layer 


\subsection{Chapter Summary}

In this chapter, the e-store system worked as a case study to illustrate how to use the detection approach presented in Chapter 4 to detect feature interactions in the Web service domain.

Parts of feature interaction categories and causes presented in the Chapter 4 also have been verified by analyzing and discussing the feature interaction examples in the e-store system. These feature interaction categories and causes could be found in other ebusiness systems.

At the end of every feature interaction example in the e-store system, a document format for feature interactions in Web services is presented. It includes feature interaction name, categories, and layers applied in the Web services stack. This document format can be a reference to document feature interactions in Web services for other researchers. The documented feature interactions can be used as a reference for similar e-business system design in the future. For example, when a system designer wants to invoke some third party Web services in his system, at the every application layer, he may always find that there are multiple services available. This will be difficult to quickly choose the appropriate services because he may not use those services before. These documents can help him to make the quickly and right choice. When he uses the documents, he can quickly locate his issues at a special application layer. Then he checks some particular services or non-functional concerns documents in this application layer. Those documents can help him to avoid some feature interactions. 


\section{Chapter 6 Conclusion and Future Work}

In this chapter, first, we will review the objectives of this thesis and summarize the contributions of this thesis. Then we will discuss possible further development for the feature interactions in Web service.

\subsection{Review of the Objectives}

The objective of this thesis is the study of feature interactions in Web services. The feature interaction problem is one of the most important problems in the telecommunication domain. Since 1992, a lot of research has been done on it [FIW92][FIW94] [FIW95] [FIW97] [FIW98] [FIW00] [FIW03]. Much research aims at how to predict, detect, and resolve feature interactions. Besides the telecommunication domain, the feature interaction problem has been found in other systems. This thesis discusses this problem in Web services.

In this thesis, UCM is used to analyze Web application system scenarios. As shown in Chapter 5 , the e-store system functional requirements have been described by UCM.

GRL, as a goal-driven analysis tool, is used to analyze the non-functional requirements of a Web service application system. In this thesis, GRL works for the feature interaction detection approach with UCM by goal-driven analysis of the system. 


\subsection{Summary of the Contributions}

In this section, a review of the claimed contributions is presented:

1) This thesis has presented eight categories of feature interaction in Web services based on three dimensions: type, scope, and role.

2) This thesis has also presented a classification of the feature interaction by causes. Every feature interaction in those eight categories may have one or more causes.

3) A further development for an existing feature interaction detection approach is also introduced. This approach is used to detect feature interaction in the Web service at the requirements stage.

4) A collection of feature interaction examples in Web services has been presented to verify categories and causes. In the appendix, an index of those examples is presented.

5) An e-store system was used as a case study to illustrate how to use the detection approach. It also verified the categories and causes presented.

6) This thesis discussed why GRL and UCM could be used to detect feature interactions in Web services. It also presented and explained how to do it.

7) Although this thesis presented three dimensions, it is unknown whether other dimensions exist. If some new dimensions can be found, the number and complexity of categories will have a large increase. In this thesis, although every category and every cause at least has an example to support it, it does not mean all relationships between categories and causes are covered. In fact, we do not know whether there are relationships between some categories and causes. 


\subsection{Future Work}

The results of this thesis provide a basis for several future reseach directions.

1) The examples in this thesis cover parts of relationships between categories and causes. That is why these are some empty slots in Table 4.2 and Table 4.3 in Chapter 4. More examples and research are needed to cover all the possible relationships between categories and causes.

2) In Chapter 5, we empirically validated that the detection approach could detect feature interactions that occurred at the requirements stage in the e-business system. It is not surprising that it can be used to detect feature interactions at the requirements stage in other systems because GRL and UCM are used in other systems. For example, the combination of GRL and UCM was used to model and refine the architecture design in the mobile telecommunication systems domain [L\&Y01]. This proves the features in the mobile telecommunication systems domain can be modeled by GRL and UCM. If features can be modeled, the feature interactions can be detected by the approach by analyzing goals and scenarios.

3) The approach for feature interaction detection is limited to the requirements stage in the Web service domain. It is a static and offline detection approach. It cannot be used to detect the feature interactions occurred at the other stages in the software lifecycle. It also cannot be used to detect the feature interactions occurred at the runtime environment. So more approaches for detecting feature interaction problems at the different stages in the software lifecycle and at the runtime environment are needed. 
4) One Web service is a black box to another Web service except input and output parameters. It is unpredictable whether there is a feature interaction if we compose them into a system. This may increase the difficulty to detect the feature interaction between them. It may be done by dynamic detection approach. However, if a feature interaction document exists between these two Web services, the feature interaction can be avoided by choosing other Web services. 


\section{Appendix: The Index of Feature Interactions in Web Services}

\begin{tabular}{|c|c|c|c|c|}
\hline Examples & Page & Canses & Categories & Description \\
\hline E4-1 & 50 & VAS & FISR & Transaction Management vs. Logging \\
\hline E4-2 & 53 & VAS & FIMR & Logging for Billing vs. Cache \\
\hline E4-3 & 54 & DIO & FIMR & ContentGenerator vs. Spellchecker \\
\hline E4-4 & 57 & $\begin{array}{c}\text { VAS, } \\
\text { EIH }\end{array}$ & FESR & Search Request Cache vs. Book Search \\
\hline E4-5 & 60 & DO & NFIMR & Manageability vs. Trust \\
\hline E5-1 & 87 & GC,DO & NFEMR & Usability vs. Privacy \\
\hline E5-2 & 100 & VAS,GC & NFIMR & Interoperability vs. Maintainability \\
\hline E5-3 & 100 & GC,DO & NFIMR & Maintainability vs. Performance \\
\hline E5-4 & 107 & RC,GC & NFIMR & Maintainability vs. Performance \\
\hline E5-5 & 115 & GC,PC & NFISR & Profitability vs. Correctness \\
\hline E5-6 & 116 & VAS & FESR & TaxCalculating vs. Shipping Method \\
\hline E5-7 & 117 & VAS,GC & NFESR & Correctness vs. Usability \\
\hline E5-8 & 117 & VAS,GC & NFEMR & Correctness vs. Usability \\
\hline
\end{tabular}

Notes:

\section{Causes:}

VAS- Violation of Assumptions about Service; DIO - Different Invocation Order; RC - Resource Contention; GC-Goal

Conflicts; PC-Policy Conflicts; DO-Deployment and Ownership; EIH - Encapsulation and Information Hiding

\section{Categories:}

FISR - Functional-Internal-SingleRole Feature Interactions

FIMR - Functional-Internal-MultipleRoles Feature Interactions

FESR-Functional-External-SingleRole Feature Interactions

FEMR-Functional-External-MultipleRoles Feature Interactions

NFISR - Non-Functional-Intemal-SingleRole Feature Interactions

NFIMR-Non-Functional-Internal-MultipleRoles Feature Interactions

NFESR-Non-Functional-External-SingleRole Feature Interactions

NFEMR--Non-Functional-External-MultipleRoles Feature Interactions

\section{Examples:}

E4-1-Example 4.1; E4-2-Example 4.2; E4-3-Example 4.3; E4-4-Example 4.4; E4-5-Example 4.5

E5-]-Example 5.1; E5-2-Example 5.2; E5-3-Example 5.3; E5-4-Example 5.4; E5-5-Example 5.5

E5-6-Example 5.6; E5-7-Example 5.7; E5-8-Example 5.8 


\section{Bibliography}

[Aalst03] W.M.P. van der Aalst, "Don't go with the flow: Web services composition standards exposed", 2003

http://tmitwww.tm.tue.nl/research/patterns/download/ieeewebflow.pdf

[Aalst04] W.M.P. van der Aalst, M. Dumas, A.H.M. ter Hofstede,

"Web Service Composition Languages: Old Wine in New Bottles?", 2004

http:/tmitwww.tm.tue.nl/research/patterns/download/wscl-euromicro.pdf

[Aho98] A. Aho, S. Gallangher, N. Griffeth, C. Schell, D. Swayne, SCF3/Sculptor with Chisel: Requirements Engineering for Communications Services, In: Proc. Of Fifth Workshop on Feature Interactions and Software Systems (FI''98), pp.45-63, IOS Press 1998

[Amyot99] D. Amyot, "Use Case Maps Quick Tutorial Version 1.0". http://www.usecasemaps.org/pub/UCMtutorial/UCMtutorial.pdf

[Amyot00] D. Amyot, L. Charfi, N. Gorse, T. Gray, L. Logrippo, J.Sincennes, B.Stepien, and T.Ware (2000), Feature Description and Feature Interaction Analysis with Use Case Maps and LOTOS. In: Sixth International Workshop on Feature Interactions in Telecommunications and Software Systems (FIW'00), Glasgow, Scotland, UK, May 2000.

[Araujo02] I. J. Araujo, "A Design Process based on patterns and non-functional requirements", master thesis, 2002. Carleton University

[A\&L\&B\&G99] D. Amyot, L. Logrippo, R.J.A. Buhr, and T. Gray (1999), Use Case Maps for the Capture and Validation of Distributed Systems Requirements. In: Fourth International Symposium on Requirements Engineering (RE'99), Limerick, Ireland, June 1999.

[Bellcore91] Bellcore Technical Advisory TANWT-001123, "Advanced Intelligent Network (AIN) Release 1, Switching System Generic Requirements [R]". 1991.

[BPEL4WS1.1] BPEL4WS1.1 Web site. "Specification: Business Process Execution Language for Web Services Version 1.1". 05 May, 2003.

http://www-106.ibm.com/developerworks/library/ws-bpel/

[BPMI04] BPMI Web site, http://www.bpmi.org/specifications.esp

[BPML02] BPML Web site. A. Arkin et al. Business Process Modeling Language (BPML), Version 1.0, 2002.

[BPSS02] P. Malu, J.J. Dubray, A. Lonjon et al. ebXML Business Process 
Specification Schema (BPSS), Version 1.05, 2002.

[Bruin00] H. de Bruin (2000), Scenario-Based Analysis of Component Compositions. In: Generative and Component-Based Software Engineering (GCSE'00), LNCS 2177.

[Buhr96] R.J.A. Buhr, R.S. Casselman, Use Case Maps for Object-Oriented Systems, Prentice Hall, 1996.

[Buhr98] R.J.A. Buhr, D. Amyot, M. Elammari, D. Quesnel, T.Gray, and S. Mankovski (1998), Feature-Interaction Visualization and Resolution in an Agent Environment. In: Kimbler, K. and W. Bouma (eds.), Fifth International Workshop on Feature Interactions in Telecommunications and Software Systems (FIW'98), IOS Press, Amsterdam, Netherlands, pp. 135-149.

[Buhr99] R.J.A. Buhr (1999), Use Case Maps as Architectural Entities for Complex Systems. In: Transactions on Software Engineering, IEEE, Vol. 24, No. 12, December 1998, pp 1131-1155.

[B\&J\&K94] J. Blom, B.Jonsson, and L. Kempe. "Using temporal logic for modular specification of telephone services". In L.G. Bouma, editor, Feature Interactions in Telecommunications Systems, pp. 178-196. IOS Press, 1994

[Calder02] M. Calder, M. Kolberg, E. H. Magill, S. Reiff-Marganiec. "Feature Interaction: A Critical Review and Considered Forecast", 2002. http://www.dcs.gla.ac.uk/ muffy/papers/calder-kolberg-magill-reiff.pdf

[Cameron94] E..J. Cameron, N. Griffeth, Y.J. Linand M.E. Nilson, W.K. Schnure and H. Velthuijsen. "A feature interaction benchmark for in and beyond". In L. G. Bouma and Hugo Velthuijsen, editors, Feature Interactions in Telecommunica tions Systems, pp.1-23, Amsterdam, 1994.IOS Press.

[Cohen02] F. Cohen, "Issues in integrating multiple vendor Web services implementations", 2002.

http:/www-106.ibm.com/developerworks/library/ws-inter.html

[C\&P94] P. Combes and S.Pickin. "Formalisation of a user view of network and services for feature interaction detection". In L.G. Bouma, editor, Feature Interactions in Telecommunications Systems, pp. 178-196. IOS Press, 1994

[C\&V93] E.J. Cameron and H.Velthuijsen, Feature Interactions in Telecommunications Systems, In: IEEE Comm., vol.31, no.8, pp.46-51, Aug.1993

[Curbera02] F. Curbera, Y. Goland, J. Klein, F. Leymann, D. Roller, S. Thatte, and S. Weerawarana. Business Process Execution Language for Web Services, Version 1.0. Standards proposal by BEA Systems, International Business Machines Corporation, and Microsoft Corporation, 2002. 
[Duclos02] F.Duclos, J.Estublier, and P.Morat. Describing and using non functional aspects in component based applications. In: Aspect-Oriented Software Development (AOSD), 2002.

http://www-adele.imag.fr/Les.Publications/intConferences/AOSD2002Duc.pdf

[EIP02] G. Hohpe (editor). Enterprise Integration Patterns. http://www.enterpriseintegrationpatterns.com, 2002.

[ESPRIT01] ESPRIT Working Group 23531, FIREworks, workshop on Language Constructs for Describing Features, 2001.

http://www.des.ed.ac.uk/home/stg/fireworks/workshop.html.

[FIW92] H. Velthuijsen, N. Griffeth, and Y.-J. Lin, editors. International Workshop on Feature Interactions in Telecommunications Software Systems, Florida, 1992.

[FIW94] L. G. Bouma and H. Velthuijsen, editors. Feature Interactions in Telecommunications Systems. IOS Press (Amsterdam), 1994.

[FIW95] K. E. Cheng and T. Ohta, editors. Feature Interactions in Telecommunications Systems III. IOS Press (Amsterdam), 1995.

[FIW97] P. Dini, R. Boutaba, and L. Logrippo, editors. Feature Interactions in Telecommunication Networks IV. IOS Press (Amsterdam), 1997.

[FIW98] K. Kimbler and L. G. Bouma, editors. Feature Interactions in Telecommunications and Software Systems V. IOS Press (Amsterdam), 1998.

[FIW00] M. Calder and E. Magill, editors. Feature Interactions in Telecommunications and Software Systems VI. IOS Press (Amsterdam), 2000.

[FIW03] D. Amyot and L. Logrippo, editors. Feature Interactions in Telecommunications and Software Systems VII. IOS Press (Amsterdam), 2003.

[Fraser99] T.Fraser, L.Badger, and M.Feldman. "Hardening COTS software with generic software wrappers". In: IEEE Symposium on Security and Privacy, pp. 2-16,1999.

[Ghosh01] A. K. Ghosh: Security \& Privacy for E-Business. John Wiley \& Sons, (2001).

[Gib01] P. Gibson, "Formal requirements models: simulation, validation and verification", Technical Report: NUI Maynooth, Computing Science Department, NUIMCS-TR-2001-02, Year 2001.

[Gib97] J.P. Gibson. "Feature requirements models: Understanding interactions". In P.Dini, R.Boutaba, and L. Logrippo, editors, Feature Interactions in Telecommunications and Software Systems $I$, pp. 46-60, IOS Press, Amsterdam, Netherlands, 1997 
[IBM03] IBM Web site. "Custom design: Select a Non-Functional Requirement", http:/www-106.ibm.com/developerworks/pattems/edge/select-application-topology.html

[ITU-T92] ITU-T Recommendation Q. 1201. "Principles of Intelligent Network Architecture [S]", Geneva, 1992.

[Kormann00] D. P. Kormann and A.D. Rubin. AT\&T Labs - Research. "Risks of the Passport Single Signon Protocol". http://avirubin.com/passport.html

[K\&K98] D. O. Keck and P. J. Kuehn, "The Feature and Service Interaction Problem in Telecommunications Systems: A Survey", IEEE Comm., Transactions on Software Engineering, Vol.24, no. 10, Oct.1998

[Liu01] L. Liu et al., GRL and OME (since 2001). http://www.cs.toronto.edu/km/GRL/

[L\&Y01] L. Liu, E. Yu. "From Requirements to Architectural Design-Using Goals and Scenarios", 2001

[Martin01] J. Martin, "Web Services: The Next Big Thing", 2001. XMLJournal

2. http://www.sys-con.com/xml/

[M\&A01] G.Mussbacher, D.Amyot (2001), A Collection of Patterns for Use Case Maps. In: First Latin American Conference on Pattern Languages of Programming (SugarLoafPLoP 2001), Rio de Janeiro, Brazil, October 2001. (Revised in January 2002)

[M\&A\&H04] M. Solanki, A. Cau, H. Zedan. "Introducing Compositionality in Web service Descriptions". 10th International Workshop on Future

Trends in Distributed Computing Systems FTDCS 2004 IEEE, Suzhou, China, May 26-28, 2004, 7 pages

http://www.cse.dmu.ac.uk/monika/Papers/Mypapers/solankim compositionality.pdf

[Nakamura00] M.Nakamura, J.Hassine, L.Logrippo. "Feature Interaction Filtering with Use Case Maps at Requirements Stage". In: M.Calder and E.Magill, Sixth International Workshop on Feature Interactions in Telecommunications and Software Systems, pp. 163-178, IOS Press, Glasgow 2000

[P3P98] P3P Web site. http://www.w3.org/TR/1998/NOTE-P3P10-Protocols-19980324

[Passport04] Passport Web site. http://support.microsoft.com/default.aspx?pr=netpass

[PerfectXml] PerfectXml Web site. http:/www.perfectxml.com/glossary4.asp

[Pulvermuller01] E. Pulvermuller, A. Speck, J.O. Coplien, M. D'Hondt, W. DeMeuter, editors. "Feature Interaction in Composed System", 2001. http://www.info.unikarlsruhe.de/ pulvermu/workshops/ecoop2001/proceedings/FICS2001.pdf 
[Roman01] E.Roman, S.Ambler, and T.Jewell. Mastering Enterprise JavaBeans. Wiley, 2001 .

[RosettaNet03] RosettaNet Web site. http:/www.rosettanet.org/RosettaNet/

[Slemko01] M. Slemko, "Microsoft Passport to Trouble". http://alive.znep.com/-marcs/passport/index.html

[SOAP04] SOAP Web site, http://www.w3.org/TR/soap/

[Solanki04] M. Solanki, A. Cau, H. Zedan. "Augmenting Semantic Web

Service Description With Compositional Specification". The 13th

International World Wide Web Conference (Refereed Semantic Web

track) WWW 2004 ACM, NYC- USA May 17-22, 2004

http://www.cse.dmu.ac.uk/monika/Papers/Mypapers/313-solanki.pdf

[Sumra03] R. Sumra, D. Arulazi. "Quality of Service for Web Service-Demystification, Limitations, and Best Practices for performance", 2003, http://www.developer.com/services/print.php/2248251

[Systinet] Systinet Web site, http://www.systinet.com/

[Tocher89] A.J. Tocher. "LOTOS and the Formal Specification of Communication Standards: An example. Formal Methods: Theory and Practice," Chapter 2, edited by P.N. Scarbach.BP Research, 1989.

[UCM03] Use Case Maps Web Page and UCM User Group, 2003.

http://www.usecasemaps.org/aboutucms.shtml

[UDDI04] UDDI Web site. http://www.uddi.org/

[URN03] International Telecommunications Union (2003), Recommendation Z.150 (02/03): User Requirements Notation (URN)-Language requirements and framework. Geneva, Switzerland. http://www.UserCaseMaps.org/urn/

[Velthuijsen93] H.Velthuijsen. "Distributed AI for Run-Time Resolution of Feature Interactions," IEEE Comp., Aug. 1993

[W3C04] Web Services Architecture. W3C,2004.

http://www.w3.org/TR/ws-arch/

[Weiss03] M. Weiss. "Feature Interactions in Web Services". In Feature Interactions in Telecommunications and Software Systems VII ( FIW'03), Ottawa, 2003 
[Wohlstadter03] E.Wohlstadter, S.Jackson, and P.Devanbu. "Dado: Enhancing middleware to support cross-cutting features in distributed, heterogeneous systems." In International Conference on Software Engineering ICSE, 2003. http:/www.cs.ucdavis.edu/ devanbu/icse2003-dado.pdf

[Wohlstadter04] E. Wohlstadter, S. Tai, T. Mikalsen, I. Rouvellou, and P. Devanbu. "GlueQoS: Middleware to Sweeten Quality-of-Service Policy Interactions". In International Conference on Software Engineering ICSE 2004. http://www.research.ibm.com/AEM/pubs/GlueQoS icse2004.pdf

[WSCI02] WSCI Web site. Web Service Choreography Interface (WSCI) 1.0. Standards proposal by BEA Systems, Intalio, SAP, and Sun Microsystems, 2002. http://www.w3.org/TR/wsci/

[WSDL04] WSDL Web site, http://www.w3.org/TR/wsdl/

[WSFL01] F. Leymann. Web Services Flow Language, Version 1.0, 2001. http://www-306.ibm.com/software/solutions/webservices/pdf/WSFL.pdf

[W\&E04] M. Weiss, B. Esfandiari. "On Undesirable Feature Interactions Among Web Services". In International Conference on Web Services ICWS 2004.

[XLANG01] S. Thatte. XLANG Web Services for Business Process Design, 2001. http://www.gotdotnet.com/team/xml wsspecs/xlang-c/default.htm

[Z151] URN Focus Group (2003), Draft Rec. Z.151- Goal-oriented Requirement Notation (GRL). Geneva, Switzerland, September 2003.

[Z152] URN Focus Group (2003), Draft Rec. Z.152-Use Case Map Notation (UCM). Geneva, Switzerland, September 2003.

[Zave99] P. Zave, "FAQ Sheet on Feature Interaction", 1999. http://www.research.att.com/ pamela/faq.html 